University of Louisville

ThinkIR: The University of Louisville's Institutional Repository

Electronic Theses and Dissertations

$5-2012$

\title{
A spatial age-structured model of perennial plants with a seed bank.
}

Kimberly llene Meyer

University of Louisville

Follow this and additional works at: https://ir.library.louisville.edu/etd

\section{Recommended Citation}

Meyer, Kimberly llene, "A spatial age-structured model of perennial plants with a seed bank." (2012). Electronic Theses and Dissertations. Paper 970.

https://doi.org/10.18297/etd/970

This Doctoral Dissertation is brought to you for free and open access by ThinkIR: The University of Louisville's Institutional Repository. It has been accepted for inclusion in Electronic Theses and Dissertations by an authorized administrator of ThinkIR: The University of Louisville's Institutional Repository. This title appears here courtesy of the author, who has retained all other copyrights. For more information, please contact thinkir@louisville.edu. 


\title{
A SPATIAL AGE-STRUCTURED MODEL OF PERENNIAL PLANTS WITH A SEED BANK
}

By

Kimberly Ilene Meyer

B.S., University of Louisville, 2007

M.A., University of Louisville, 2008

\author{
A Dissertation \\ Submitted to the Faculty of the \\ College of Arts and Sciences of the University of Louisville \\ in Partial Fulfillment of the Requirements \\ for the Degree of
}

Doctor of Philosophy

Department of Mathematics

University of Louisville

Louisville, $\mathrm{KY}$

May 2012 
Copyright 2012 by Kimberly Ilene Meyer

All rights reserved 


\title{
A SPATIAL AGE-STRUCTURED MODEL OF PERENNIAL PLANTS WITH A SEED BANK
}

\author{
Submitted by \\ Kimberly Ilene Meyer \\ A Dissertation Approved on

$\frac{2 / 29 / 12}{\text { (Date) }}$

by the Following Reading and Examination Committee:

$\frac{\text { Bingtuan Li, Dissertation Director }}{\text { Jiaxu Li }}$

Ryan Gill

Changbing $\mathrm{Hu}$

Susanna Remold 


\section{ACKNOWLEDGMENTS}

I would like to thank my dissertation advisor, Dr. Bingtuan Li, for his guidance, patience, encouragement and leadership. It has been a priviledge to collaborate with him and have him as a mentor. I thank my committee members for their support and encouragement throughout.

I wish to thank my family for providing me with unconditional love and unsurpassed support. In particular, I thank my mom, Michele Meyer, for her historic words of wisdom "Listen to your teacher, do your best and have fun!" which have guided me throughout my education. I thank my dad, Dick Meyer, for igniting my passion for mathematics at a very young age by revealing the beauty of the variable $x$. I thank my brothers, Matt and Jonny Meyer, and my boyfriend, Corey Valentine, for being my best friends and always believing in me. 


\section{ABSTRACT \\ A SPATIAL AGE-STRUCTURED MODEL OF PERENNIAL PLANTS WITH A SEED BANK

\author{
Kimberly Ilene Meyer
}

May 12, 2012

We formulate an integro-difference model to predict the growth and spatial spread of a perennial plant population with an age-structured seed bank. We allow the seeds in the bank to be of any age, producing an infinite system of equations. The production of new seed can be density-dependent and so the function describing this growth is allowed to be non-monotone. The functions describing the seed bank are linear. We develop properties about the non-spatial model, including the existence of a positive steady-state and conditions under which solutions converge to this steady-state. We also show that when the origin is unstable, the system has a spreading speed and that this spreading speed is characterized as the slowest speed of a class of traveling wave solutions. We conduct numerical simulations of a truncated version of this model which show that both the perennial term and the sced bank can have a stabilising effect on the population. On the other hand, traveling wave solutions may exhibit different patterns of fluctuations including periodic oscillations and chaotic tails. 


\section{TABLE OF CONTENTS}

\section{CHAPTER}

1. INTRODUCTION AND BACKGROUND . . . . . . . . . . . . 1

1.1 Introduction . . . . . . . . . . . . . . 1

1.2 Difference Equations . . . . . . . . . . . . . . . . . . 3

1.3 Integro-Difference Equations . . . . . . . . . . . . . . 9

2. THE MODEL ............................. 15

2.1 The Growth Equations . . . . . . . . . . . . . . . . 15

2.2 The Spatial Model . . . . . . . . . . . . . . . . 21

3. DIFFERENCE EQUATION ANALYSIS . . . . . . . . . . . . . . 24

3.1 Existence of Eigenvalue and Eigenvectors . . . . . . . . . 26

3.2 Steady-States . . . . . . . . . . . . . . . 28

3.2.1 The Extinction Steady-State . . . . . . . . . . 29

3.2.2 Existence of a Positive Steady-State . . . . . . . . 30

3.2.3 Convergence to the Positive Steady-State . . . . . . . 32

3.2.4 Connection to Analogous Finite System . . . . . . . . 37

3.3 Solution Curves and Bifurcation Diagrams . . . . . . . . 38

4. SPATIAL ANALYSIS . . . . . . . . . . . . . . . . 42

4.1 Existence of the Eigenvalue . . . . . . . . . . . . 47

4.2 Properties of the Eigenvalue . . . . . . . . . . . . . 49

4.3 The Spreading Speed . . . . . . . . . . . . . . . 54

4.4 Traveling Wave Solutions . . . . . . . . . . . . . 60

5. NUMERICAL SIMULATIONS ................. 75 
6. CONCLUSION AND FUTURE DIRECTIONS . . . . . . . . . . . 84

6.1 Conclusion . . . . . . . . . . . . . . . . 84

6.2 Future Directions . . . . . . . . . . . . . . . . . . . 86

REFERENCES . . . . . . . . . . . . . . . . . . . . . 88

CURRICULUM VITAE . . . . . . . . . . . . . . . . . . . 93 


\section{LIST OF FIGURES}

$1.1 \quad$ The Ricker function, $f(u)=u e^{r-u}$, where $r=1 \ldots 5$

1.2 Solutions to the Ricker function with $u_{0}=0.2$ and $r$ varies . . 6

1.3 Bifurcation diagram of the Ricker function . . . . . . . 7

1.4 Generic plot of the possible wave speeds (c) versus possible wave shapes $(\mu)$. The spreading speed $c^{*}$ has been indicated as the infimum over $\mu$ in both plots. . . . . . . . . . 12

2.1 Life-cycle of Perennial Plant Population with a Seed Bank. . . 18

$3.1 \quad$ Solution curves of $(3.1)$ with $H$ the Ricker function. . . . . . . 39

3.2 The bifurcation diagrams of a truncated version of (3.1) with $H$

the Ricker function and $r$ the bifurcation parameter, $r \in[0,15] .40$

3.3 The bifurcation diagrams of a truncated version of (3.1) with

$H$ the Ricker function and the new seed germination rate $\beta_{0}$ as the bifurcation parameter, $\beta^{0} \in[0,1] \ldots \ldots \ldots$. . . . . 41 
5.1 Role of Germination Rate Numerical simulations for the model with $r=1(\mathrm{a}, \mathrm{b}, \mathrm{c}), r=3(\mathrm{~d}, \mathrm{e}, \mathrm{f})$, and $r=6(\mathrm{~g}, \mathrm{~h}, \mathrm{i})$. Vertically the simulations represent: (1) Leftmost (a, d, g) traveling waves when the germination rate increases with age, $\beta^{0}=0.025, \beta^{1}=0.05, \beta^{2}=0.1, \beta^{3}=0.2,(2)$ Center $(b, e$, h) - traveling waves when the germination rate decreases with age, $\beta^{0}=0.2, \beta^{1}=0.1, \beta^{2}=0.05, \beta^{3}=0.025$, (3) Rightmost $(c, f, i)$ - traveling waves when the germination rate fluctuates with age, $\beta^{0}=0.05, \beta^{1}=0.1, \beta^{2}=0.2, \beta^{3}=0.025$. All other parameters take the following values: $\rho_{o}=0.5, \rho_{d}^{0}=\rho_{d}^{1}=\rho_{d}^{2}=$ $\rho_{d}^{3}=1, \rho_{\nu 1}=\rho_{\nu 2}=0.9 \ldots \ldots \ldots \ldots \ldots$

5.2 No Seed Bank Numerical simulations for the model when $s_{i}=0$ for $i=0,1,2 \ldots \ldots \ldots \ldots$. . . . . . . . 82

5.3 Seed Bank Numerical simulations for the model with an agestructured seed bank for seeds up to age 2: $A(x)=$ black line, $S^{0}(x)=$ blue line, $S^{1}(x)=$ green line, $S^{2}(x)=$ red line. $\ldots .83$ 


\section{CHAPTER 1 INTRODUCTION AND BACKGROUND}

\subsection{Introduction}

Dispersal of seed in plant populations can play a major role in the population dynamics and spatial spread of a species [3]. This process is difficult to quantify and thus, historically, population models tend to ignore dispersal $[28,40,42,45]$. In the past several decades integro-difference equations have emerged as a means to study spatial spread of ecological populations $[1,2,9$, $10,12,14,16,17,18,31,32,43]$, among other applications. The main function of these types of models is to elucidate dispersal patterns by quantifying the rate of spread of populations and investigating traveling wave solutions. For more details and historical development of traveling waves see $[16,22,23,24,25,26,27,47]$.

In this dissertation we model the population dynamics and spatial spread of perennial plants with a seed bank. Perennial (and annual) plants have one reproductive season per year during which they allocate some of their resources to produce seed. This seed is then subject to passive dispersal by means of water, wind, or adherence to an active agent, eventually settling and burying in the soil. Once a seed has been dispersed and buried, it undergoes a period of dormancy, potentially leading to germination and ultimately developing into a mature plant.

Many models assume seed germination occurs within 1-year of seed production [45]. However, the dormancy period for seeds can last much longer than 
one year, effectively creating a delay in the development of generations. Seeds persisting in dormancy for more than one year comprise what is known as the seed bank.

The longevity and viability of seeds in the seed bank are dependent upon the type of species, availability of resources, and natural environmental conditions. Seed banks may contain just a few hundred to over 100,000 seeds per square meter [8] and seeds may persist for hundreds or even thousands of years [11]. This type of delay in germination can naturally have consequences on the overall population dynamics of the plant as well as the spatial spread of the population.

Seed bank models have been constructed in both a spatial $[1,31,35,36]$ and non-spatial context $[5,8,28,38,40,42]$. MacDonald \& Watkinson [28] studied non-spatial difference equation models and showed that a seed bank can have both a stabilizing and destabilizing effect on an annual plant population. Allen, Allen \& Gilliam [1] construct a similar model but apply a spatial aspect using integrodifference equations to describe the dispersal of seed. They found a formula for the spreading speed of populations described by this model but did not prove existence of traveling wave solutions. They also considered spatial spread for perennial plants but did not include a seed bank.

Schmidt \& Lawlor [40] consider seed bank models for annual plants in which all age classes of seeds could have different germination rates. These models take the form of structured Leslie matrix equations. They found that sensitivity of the growth rate to germination fractions was smaller than the sensitivity to fecundity and survivorship.

Neubert \& Caswell [31] study more general matrix models and apply a spatial aspect using integro-difference equations. They develop a recipe for calculating the sensitivity and elasticity of the spreading speed to changes in the demographic and dispersal parameters. They give a formula for the spreading speed but do not 
consider existence of traveling waves.

In this dissertation we construct a novel spatial model for perennial plants with an age structured seed-bank in the form of integro-difference equations. This model has the benefit of synthesizing and generalizing many of the aforementioned models, while providing a clear focus on perennial plants.

In the construction of our growth equations we allow for density-dependent growth (a fundamental premise in plant population growth [13]). We include a term which describes the survivorship of adults from generation to generation which distinguishes this model from that of an annual plant. We also assume the rate of seed germination is dependent on the age of the seed.

In this chapter we aim to give the reader a brief but sufficient background on difference equations and integro-difference equations so that the core content and results of this dissertation are accessible. Section 1.2 introduces the concept of a difference equation and the typical methodology employed to analyze and study them. We provide an example using the Ricker function which will be used for numerical simulations in later chapters. Section 1.3 introduces integro-difference equations. We describe how they are developed and discuss spreading speeds and traveling wave solutions.

\subsection{Difference Equations}

Difference equations are, very simply, recursive functions in which successive inputs depend on the previous output. They are used to model discrete processes over time wherein future states depend on the current state. We may represent this dependence by using nonnegative integer indices $n$ to distinguish the discrete state of each solution

$$
u_{n+1}=f\left(u_{n}\right)
$$


Given an initial condition, $u_{0}$, The function $\int$ takes the $n$-th solution of the recursion as an input and produces the $n+1$-th solution. This iterative process produces a solution sequence $\left\{u_{i}: i=0 \ldots \infty\right\}=\left\{u_{0}, u_{1}, \ldots, u_{n}, \ldots\right\}$.

When the equation (1.1) is assigned meaning, one would be interested to know how this solution sequence can be interpreted in the real world. For the purposes of this dissertation, we consider growth dynamics of plant populations. In the case of a seasonally reproductive plant, the plant population next year will depend on the number of seeds produced by the population this year. The dependence of next years population on the current years population makes this situation a perfect candidate to be modeled using difference equations.

In this context, the variable $u_{n}$ in function (1.1) represents the plant population at time $n$ and the function $f$ determines how the population will change over time. Thus, the solution sequence produced tells us how the population fluctuates over time.

In many cases there is a tendency for the population to increase when numbers are small however as the population becomes too large for its environment the population decreases. The Ricker function is a well studied difference equation used to model this so called 'density dependence' and we will use this function throughout the dissertation to produce numerical simulations.

The Ricker function takes the form

$$
u_{n+1}=u_{n} e^{r-u_{n}}
$$

where $r$ is a parameter related to the growth rate of the population.

Figure 1.1 depicts a continuous plot of the Ricker function $f(u)=u e^{r-u}$ to highlight the general humped shape of this function (we use $r=1$ for this plot). The parameter $r$ controls the steepness of the hump which always attains its maximum at 1 . Notice that, initially, this function behaves like an exponential 


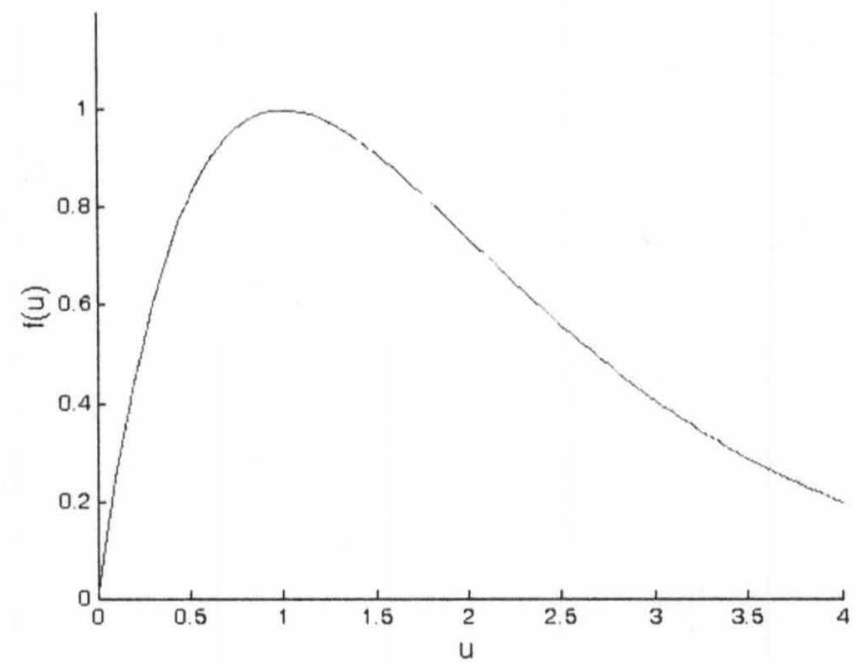

FIGURE 1.1-The Ricker function, $f(u)=u e^{r-u}$, where $r=1$

function but once the population gets too large it maxes out and begins to decline.

Depending on the choice of initial condition and choice of parameter $r$, equation (1.2) can have wildly varying solution sequences including stable solutions, periodic oscillations, and even chaotic solutions [29, 30]. Figure 1.2 displays 4 solution curves to the Ricker function for various values of $r$. We note that as $r$ increases, the solutions become more complex.

Another way to numerically visualize the wide spectrum of solutions that can be produced from a difference equation is to observe a bifurcation diagram. A bifurcation diagram is a plot of the long-term solutions of a function for various values of a bifurcation parameter.

Figure 1.3 is a bifurcation diagram of the Ricker function (1.2) with $r$ as the bifurcation parameter and initial condition $u_{0}=0.2$. For values of $r$ between 0 and 5 incremented by 0.002 , we calculate the first 100 solutions and plot solutions 91 to 100. From this diagram we observe that the Ricker Function has stable solutions for $0 \leq r \leq 2$, moving into cascades of period doubling and finally displaying 


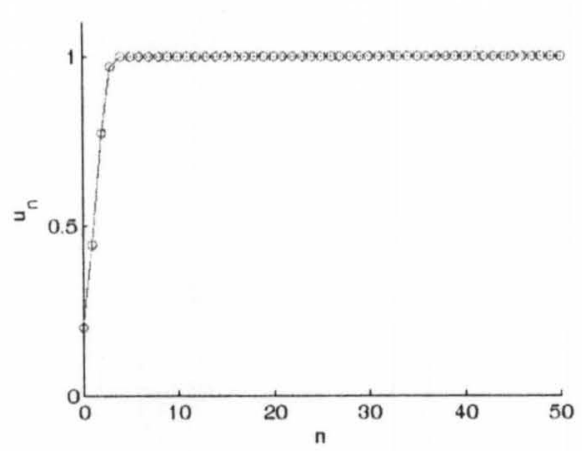

(a) $r=1$, Stable Solutions

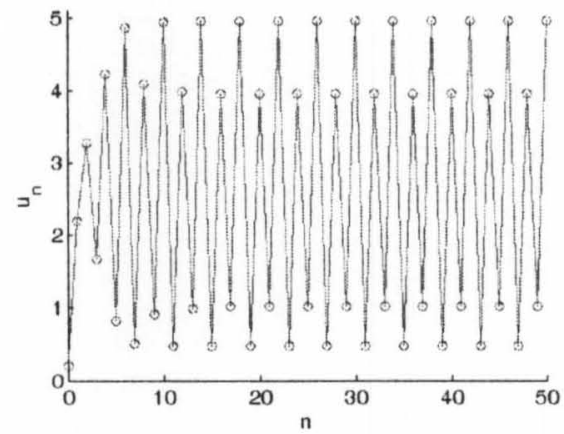

(c) $r=2.6$, Period 4 Oscillations

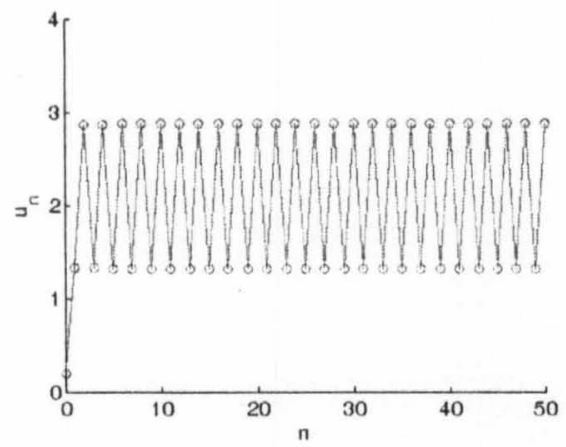

(b) $r=2.1$, Period 2 Oscillations

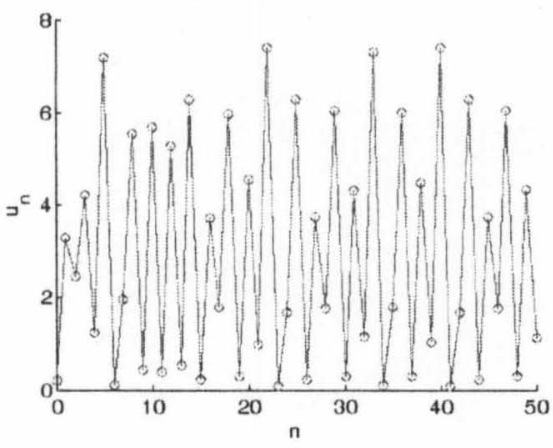

(d) $r=3$, Chaos

FIGURE 1.2 -Solutions to the Ricker function with $u_{0}=0.2$ and $r$ varies

chaotic solutions for large values of $r$.

Another well received density-dependent function that is used to model growth in biological populations is the Beverton-Holt function [4]:

$$
f(u)=\frac{R u}{1+u / M}
$$

where $R>1$ is the per capita growth rate and $K=(R-1) M$ is the carrying capacity of the environment. This is an increasing function which approaches the horizontal asymptote $R M$ as $u \rightarrow \infty$. 


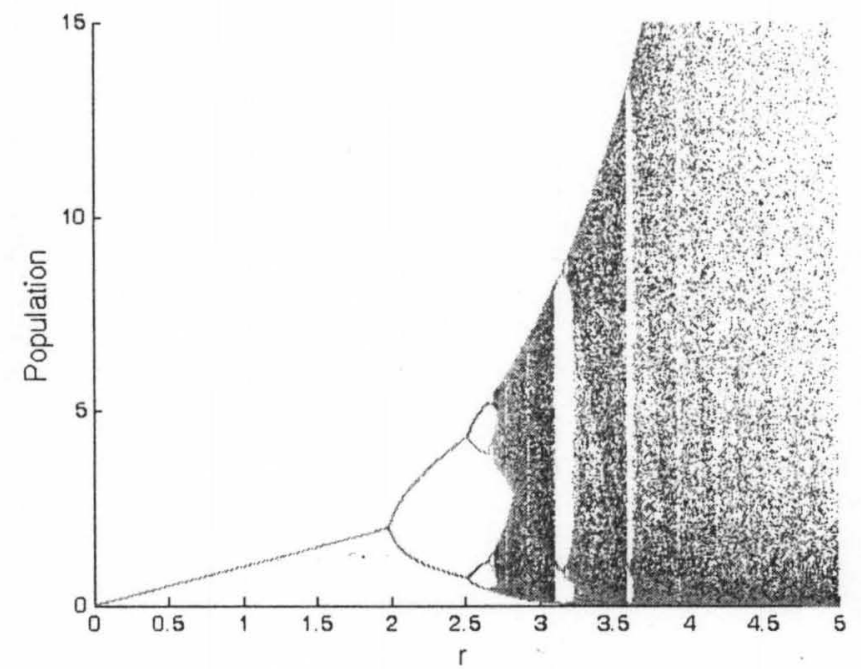

FIGURE 1.3-Bifurcation diagram of the Ricker function 


\section{Structured Difference Equation Models}

While difference equation (1.1) is suitable to model population growth for a simplistic situation, it does not address the effect of demographic variances in a population such as size, age and developmental stage. These variances affect an individual's vital rates and response to the environment and thus affect population dynamics [31].

In order to incorporate demographic variances in the model (1.1) we introduce structure in the form of an $m$-dimensional system

$$
\begin{aligned}
u_{n+1}^{1}= & f_{1}\left(u_{n}^{1}, u_{n}^{2}, \ldots, u_{n}^{m}\right) \\
u_{n+1}^{2}= & f_{2}\left(u_{n}^{1}, u_{n}^{2}, \ldots, u_{n}^{m}\right) \\
& \vdots \\
u_{n+1}^{m}= & f_{m}\left(u_{n}^{1}, u_{n}^{2}, \ldots, u_{n}^{m}\right)
\end{aligned}
$$

where $u_{n}^{i}$ is the $i$-year old population density at time $n$ and $f_{i}$ determines how the $i$-year old population density fluctuates over time.

We may write this system in the more succinct vector form

$$
\mathbf{u}_{n+1}=\mathbf{f}\left(\mathbf{u}_{n}\right)
$$

Specifically, we are interested in the effect of age on population dynamics. A popular and well studied age-structured model is the Leslie model which only considers births and deaths amongst the age classes. This model takes the form of a matrix population model

$$
\mathbf{u}_{n+1}=\mathbf{L} \mathbf{u}_{n}
$$

where 


$$
\mathbf{L}=\left(\begin{array}{ccccc}
r_{11} & r_{12} & \ldots & r_{1, m-1} & r_{1 m} \\
t_{21} & 0 & \ldots & 0 & 0 \\
0 & t_{32} & \ldots & 0 & 0 \\
\vdots & \vdots & \ddots & \vdots & \vdots \\
0 & 0 & \ldots & t_{m, m-1} & 0
\end{array}\right)
$$

is an $m \times m$ matrix containing the age-specific reproduction and survival rates. The scalars $r_{1 j}$ represent the expected number of offspring produced per $j$-year old individual and the scalars $t_{j+1, j}$ represent the expected number of $j$-year old individuals that survive to the next year.

Given an initial population $\mathbf{u}_{0}=\left(u_{0}^{1}, u_{0}^{2}, \ldots, u_{0}^{m}\right)$, the solution of the Leslie model (1.6) is given by

$$
\mathbf{u}_{n}=\mathbf{L}^{n} \mathbf{u}_{0}
$$

It is important to note that the Leslie model (1.6) assumes births and deaths are scalar rates and thus does not allow for density dependence. Density dependence may be incorporated by generalizing this model to allow the processes of birth and death to be non-linear functions instead of scalars.

\subsection{Integro-Difference Equations}

Difference equations provide an excellent means to study growth dynamics of populations with discrete generations however this type of model does not account for migration of a population in space. In the past several decades integrodifference equations have emerged as a means to model and study dispersal of populations.

To develop an integro-difference equation we assume there is a sedentary stage, in which the population grows, and a dispersal stage, in which the population migrates. We assume these stages occur independently with the growth occurring 
in discrete-time intervals and the migration occurring in continuous 1-dimensional space.

1. Sedentary Stage The sedentary stage is modeled with a difference equation, $u_{n+1}=f\left(u_{n}\right)$, called the growth function . As discussed in Section 1.2, $u_{n}$ represents the density of the population at time $n$ and the function $f$ can be nonlinear to account for density-dependence.

2. Dispersal Stage The dispersal stage is modeled using a probability density function $k(x)$ commonly called the dispersal kernel. The distribution $k(x-$ y) represents the likelihood that an individual migrates from location $y$ to location $x$.

Since $k(x)$ is a probability distribution we have that $k(x)$ must be nonnegative and $\int_{-\infty}^{\infty} k(x) d x=1$.

The population density at location $x$ and time $n+1$ is given by the sum of the contributions from all other locations $y$ giving the integro-difference equation:

$$
u_{n+1}(x)=\int_{-\infty}^{\infty} k(x-y) f\left(u_{n}(y)\right) d y
$$

The population is now a function of space and so for each discrete time step $n$, we have a distribution $u_{n}(x)$ representing the population density as opposed to a scalar quantity.

In Section 1.2 we added structure to the difference equation (1.1) to account for demographic variances in the population. Similarly, we may add structure to the integro-difference equation (1.8) to obtain the $m$-vector-valued system

$$
\mathbf{u}_{n+1}(x)=\int_{-\infty}^{\infty} \operatorname{diag}(\mathbf{k}(x-y)) \mathbf{f}\left(\mathbf{u}_{n}(y)\right) d y
$$

where $\mathbf{k}(x)$ is a diagonal matrix of dispersal kernels corresponding to stage-specific dispersal and $\mathbf{f}(\mathbf{u})$ describes how population densities fluctuate over time. 


\section{The Spreading Speed}

One of the motivating factors for studying dispersal is to understand the rate of spread of a population; How quickly is a population advancing or retreating in its environment? As a mechanism to quantify spread we consider the asymptotic behavior of solutions as $n$ approaches infinity. This is called the spreading speed.

For the scalar recursion (1.8) we define precisely what is meant by the spreading speed. Suppose there exists a positive steady-state $\beta$ of the recursion (1.8). Then the number $c^{*}$ is the spreading speed in the following sense:

1. If $u_{n}(x)$ is a solution of the recursion (1.8) with $0 \leq u_{0}(x)<\beta$ uniformly in $x$ and $u_{0}(x)=0$ for all sufficiently large $x$, then for any positive $\epsilon$

$$
\lim _{n \rightarrow \infty}\left\{\sup _{|x| \geq n\left(c^{*}+\epsilon\right)} u_{n}(x)\right\}=0
$$

2. For every $l$ with $0<l<1$ there exists a positive number $r_{l}$ such that if $u_{n}$ is a solution of $(1.8)$ and if $0 \leq u_{0}(x)<\beta$ and $u_{0}(x) \geq l \beta$ on an interval of length $r_{l}$, then for any positive $\epsilon$,

$$
\lim _{n \rightarrow \infty}\left\{\sup _{|x| \leq n\left(c^{*}-\epsilon\right)}\left(\beta-u_{n}(x)\right)\right\}=0 .
$$

The first statement says that $c^{*}$ is an upper bound for the spreading speed. If $c^{*}$ is, in fact, the asymptotic rate of spread then we would expect that at time $n$, the support of the solution would have grown by $n c^{*}$. Thus, points outside the expected support of the solution $\left(|x| \geq n\left(c^{*}+\epsilon\right)\right)$ should have no individuals present. Said another way, if we always move faster than the rate of spread of the population, we should always be in front of the advancing population.

Alternatively, the second statement says that $c^{*}$ is a lower bound for the spreading speed. If we always move more slowly than the population $\left(|x| \leq n\left(c^{*}-\right.\right.$ 


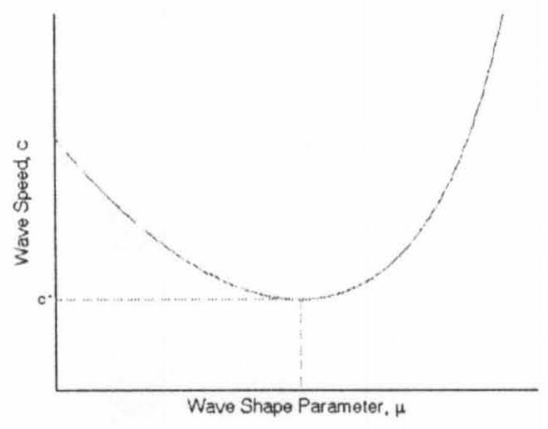

(a) Infimum attained at finite value, $\mu$

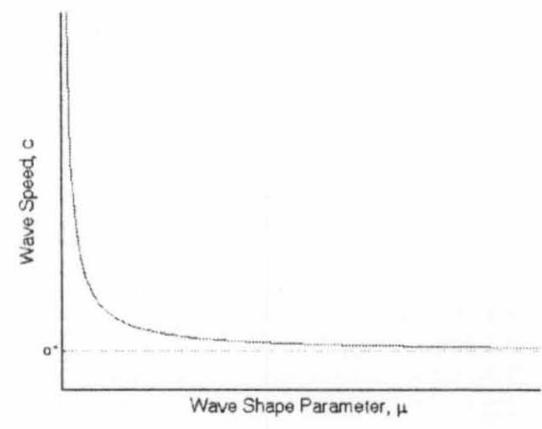

(b) Infimum attained at $\mu=\infty$

FIGURE 1.4-Generic plot of the possible wave speeds $(c)$ versus possible wave shapes $(\mu)$. The spreading speed $c^{*}$ has been indicated as the infimum over $\mu$ in both plots.

$\epsilon)$ ) then we should always be behind the leading edge of the advancing population.

In this case, over a long period of time, we should approach the steady-state $\beta$.

Together, the first and second statements describe $c^{*}$ as the asymptotic rate of spread of the solutions of the recursion (1.8).

Weinberger [46] showed that for the scalar case (1.8), under certain conditions, the spreading speed $c^{*}$ can be calculated with a formula:

$$
c^{*}=\inf _{\mu>0}\left\{\frac{1}{\mu} \ln \left(f^{\prime}(0) \int_{-\infty}^{\infty} e^{\mu y} k(y) d y\right)\right\} .
$$

The wave speed equation $\phi(\mu)=\frac{1}{\mu} \ln \left(f^{\prime}(0) \int_{-\infty}^{\infty} e^{\mu y} k(y) d y\right)$ is a convex function [27] and thus the infimum in Eq. (1.12) exists. Figure 1.4 depicts the two possible shapes that the wave speed equation can take; either the infimum is attained at a finite value as in Figure 1.4a or the infimum is attained at $\infty$ as in Figure 1.4b.

For the general case of a nonlinear multi-species system (1.9), there exists multiple spreading speeds; Different species can move at different speeds. Presently there is no known formula for the spreading speed of a nonlinear system. However, if the nonlinear system is dominated by a linear system in the direction of the 
vector corresponding to the principal eigenvalue of the generating matrix then the spreading speed of the nonlinear system is equal to that of the linear system [48]. This is known as linear determinacy.

When the nonlinear system is linearly determinate then the spreading speed formula is given by:

$$
c^{*}=\inf _{\mu>0}\left\{\frac{1}{\mu} \ln \lambda_{1}(\mu)\right\}
$$

where $\lambda_{1}$ is the principal eigenvalue of the linearized moment generating matrix

$$
\mathbf{B}_{\mu}:=\mathbf{K}(\mu) \mathbf{f}^{\prime}(\mathbf{0})
$$

Here $\mathbf{f}^{\prime}(\mathbf{0})$ is the Jacobian matrix of $\mathbf{f}\left(\mathbf{u}_{n}\right)$ (i.e. the matrix whose entries are the partial derivatives of $\mathbf{f}\left(\mathbf{u}_{n}\right)$ ) and $\mathbf{K}(\mu)$ is defined to be the moment generating matrix

$$
\mathbf{K}(\mu):=\operatorname{diag}\left(\int_{-\infty}^{\infty} \mathrm{e}^{\mu z} \mathbf{k}(z) d z\right)
$$

In the next section we will discuss how the spreading speed is related to traveling wave speeds. 


\section{Traveling Wave Solutions}

A non-constant solution of the vector-valued integro-difference system (1.9) of the form

$$
\mathbf{u}_{n}(x)=\mathbf{w}(x-n c)
$$

where $c$ is a positive constant, is called a traveling wave solution with speed $c$.

Note that traveling wave solutions are not explicitly dependent on time. Instead, they are solutions which retain their shape but are translated by a fixed length for each iteration of time. Thus, over time, the solutions travel in space at the rate of $c$.

Weinberger [46] showed that for the monotone scalar model (1.8), the spreading speed $c^{*}$, is the minimum wave speed for a class of traveling wave solutions. When $c \geq c^{*}$ there exists a traveling wave solution $w(x-n c)$ which is nonincreasing. When $c<c^{*}$ there is no traveling wave with speed $c$.

As mentioned before, multi-species systems may have different spreading speeds corresponding to different species. It has been shown that for cooperative systems and some almost cooperative systems, the slowest of these spreading speeds can be characterized as the minimum wave speed for a particular class of traveling wave solutions $[23,48]$.

The connection between spreading speeds and traveling wave speeds is useful because it is often more convenient to calculate the minimum speed for traveling waves than it is to calculate the spreading speed. 


\section{CHAPTER 2 THE MODEL}

In this chapter we introduce a spatial age-structured model for perennial plants with a seed bank in the form of an integro-difference equation system. The construction of our model can be dissected into two parts:

1. The Growth Equations: Construction of the difference equations used to describe the growth processes of a perennial plant with a seed bank.

2. The Spatial Model: Coupling the growth equations with appropriate functions to describe the dispersal of seeds in the form of integro-difference equations.

\subsection{The Growth Equations}

We begin with a general description of the life-cycle of an adult perennial plant. The entire life-cycle takes place in a year and we assume the process begins at the end of summer. Adult plants produce seed at the end of summer, the seeds lay dormant in the fall and winter, they germinate in the spring and finally become fecund adults by the beginning of the next summer. Since perennial plants can live for more than one year, also assume that a portion of the adult plants survive dormancy and are present the next year.

We call the fall and winter dormancy the dormant period, and the summer growing period the vegetative period. Seeds that don't germinate during 
the spring but survive dormant in the ground contribute to the seed bank. To investigate the effect of the seed bank on the overall adult population, we distinguish seeds in the bank by age. 'Thus, our plant population is divided into an infinite number of cohorts, one for the adult population and one for each possible age of seeds in the bank.

Let $A_{n}$ represent the density of adult plants at time $n$ and let $S_{n}^{j}$ represent the density of $j$-year old seeds in the seed bank at time $n(j, n \in\{0,1,2, \ldots\})$. The reproduction function $F$ determines the number of seeds produced per adult individual and can be nonlinear to account for density-dependence.

We assume survival over the dormant/vegetative periods and germination are scalar rates for all cohorts of the population. The Greek character $\rho$ indicates a survival rate with a subscript of $d$ signifying survival over the dormant period $\left(\rho_{d}\right)$, a subscript of $\nu$ signifying survival over the vegetative period $\left(\rho_{\nu}\right)$, and a subscript of $o$ signifying survival of adult plants over one year $\left(\rho_{o}\right)$. The Greek character $\beta$ indicates a germination probability.

We make the following assumptions:

1. Survivorship Assumptions: Assume survivorship over the dormant period is age-dependent. Alternatively, we assume the age of a germinating seed has no effect on survivorship over the vegetative period. Hence, we have only two survival rates associated with the vegetative period; one describing survival of germinated seed $\rho_{\nu 1}$ and the other describing survival of non-germinated seed $\rho_{\nu 2}$. This assumption is intuitively reasonable and has been used in other plant population models as well $[40,44]$.

2. Germination Assumptions: Assume the seed germination rates are agedependent seeing as how many physical, physiological and ecological processes related to germination are time dependent, . For example, over time, 
TABLE 2.1

Seed Bank Model - Parameter Descriptions

\begin{tabular}{ll}
\hline Parameter & Description of Parameter \\
\hline$\rho_{o}$ & probability an adult plant survives a full year \\
\hline$\rho_{d}^{j}$ & probability a $j$-year old seed survives the dormant period (fall \\
& and winter) \\
\hline$\beta^{j}$ & probability a $j$-year old seed germinates \\
\hline$\rho_{v 1}$ & probability a germinated seed survives the vegetative period \\
& (summer) \\
\hline$\rho_{v 2}$ & $\begin{array}{l}\text { probability a non-germinating seed survives the vegetative pe- } \\
\text { riod (summer) }\end{array}$ \\
\hline
\end{tabular}

germination inhibitors decay and the seed coat becomes tainted [37]. Thus, seeds of varying ages will naturally have differing rates of germination.

Table 2.1 gives a description of each parameter mentioned above. Agedependent parameters are identified with superscripts indicating the age of the cohort that the parameter relates to.

The flow-chart in Figure 2.1 depicts the life-cycle of each cohort starting at the end of summer in the beginning of year $n$ and ending at the end of summer in the beginning of year $n+1$. 


\section{Age-Structured Seed Bank Model}

Time Season
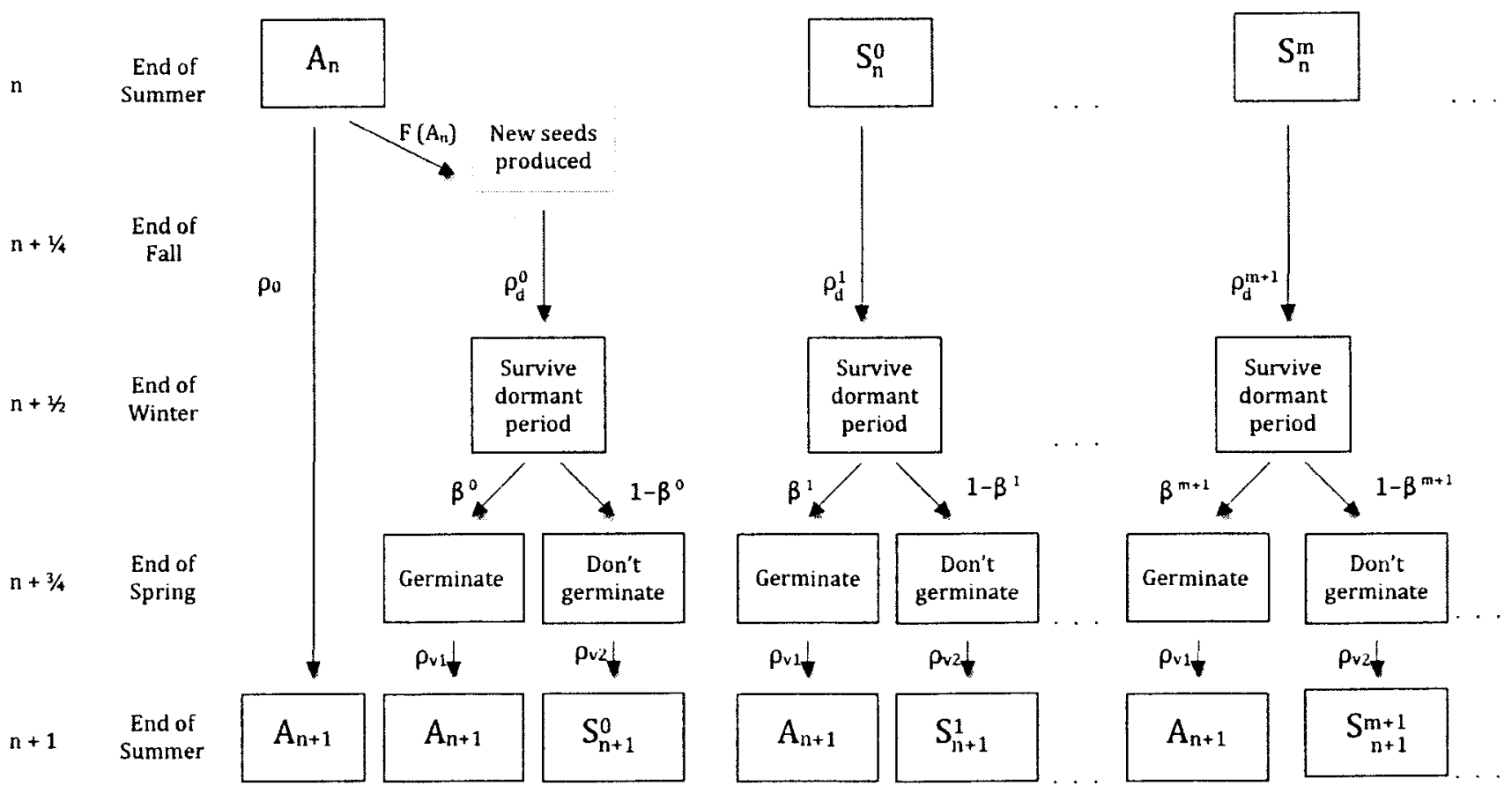

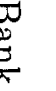


To follow the flow-chart in Figure 2.1 consider the adult population block $A_{n}$. Over the course of a year, adult plants can contribute to the next years' population in 2 ways:

1. An adult plant survives an entire year. Because we consider perennial plants, a portion $\rho_{o}$ of the adult population in year $n$ will survive a year and again be part of the adult population in year $n+1$.

2. An adult plant produces seed which can either mature into a new adult plant or survive as a seed and become part of the seed bank. First, adults produce new seed, the number of which is determined by the function $F$. A portion of these new seeds will survive the dormant period with probability $\rho_{d}^{0}$, a portion $\beta^{0}$ will germinate, and a portion $\rho_{\nu 1}$ will survive the vegetative period. These surviving plants are now mature adults and part of the adult cohort $A_{n+1}$. The portion of non-germinated seeds $\left(1-\beta^{0}\right)$ will survive the vegetative period with probability $\rho_{\nu 2}$ and become part of the 0-year old seed bank cohort $S_{n+1}^{0}$.

To observe the life-cycle of the remaining seed bank cohorts, a similar logical flow can be applied.

Figure 2.1 gives rise to the following infinite-dimensional population model describing population growth of perennial plants with an age-structured seed bank: 


$$
\begin{aligned}
A_{n+1} & =\rho_{o} \Lambda_{n}+\rho_{d}^{0} \beta^{0} \rho_{\nu 1} M\left(A_{n}\right)+\sum_{j=1}^{\infty} \rho_{d}^{j} \beta^{j} \rho_{\nu 1} S_{n}^{j-1} \\
S_{n+1}^{0} & =\rho_{d}^{0}\left(1-\beta^{0}\right) \rho_{\nu 2} H\left(A_{n}\right) \\
S_{n+1}^{1} & =\rho_{d}^{1}\left(1-\beta^{1}\right) \rho_{\nu 2} S_{n}^{0} \\
& \vdots \\
S_{n+1}^{m} & =\rho_{d}^{m}\left(1-\beta^{m}\right) \rho_{\nu 2} S_{n}^{m-1}
\end{aligned}
$$

where $I(u)=u F(u)$. We will use $H(u)$ instead of $u F(u)$ for simplicity. Letting $a_{j}=\rho_{d}^{j} \beta^{j} \rho_{\nu 1}$ and $s_{j}=\rho_{d}^{j}\left(1-\beta^{j}\right) \rho_{\nu 2}$ for all $i=0,1,2, \ldots$ the model can we written more succinctly as:

$$
\begin{aligned}
A_{n+1} & =\rho_{o} A_{n}+a_{0} H\left(A_{n}\right)+\sum_{j=1}^{\infty} a_{j} S_{n}^{j-1} \\
S_{n+1}^{0} & =S_{0} H\left(A_{n}\right) \\
S_{n+1}^{1} & =s_{1} S_{n}^{0} \\
& \vdots \\
S_{n+1}^{m} & =s_{m} S_{n}^{m-1}
\end{aligned}
$$

or in matrix form as:

$$
\left(\begin{array}{l}
A_{n+1} \\
S_{n+1}^{0} \\
S_{n+1}^{1} \\
\vdots
\end{array}\right)=\left(\begin{array}{llll}
\rho_{o}+a_{0} F\left(A_{n}\right) & a_{1} & a_{2} & \ldots \\
s_{0} F\left(A_{n}\right) & 0 & 0 & \ldots \\
0 & s_{1} & 0 & \cdots \\
\vdots & \vdots & \vdots & \ddots
\end{array}\right)\left(\begin{array}{c}
A_{n} \\
S_{n}^{0} \\
S_{n}^{1} \\
\vdots
\end{array}\right)
$$

These new parameters $\left(a_{j}, s_{j}\right)$ are a product of survival and germination rates and may be interpreted as the age-dependent probabilities of contribution to either the 
TABLE 2.2

Seed Bank Model - Simplified Parameter Descriptions

\begin{tabular}{ll}
\hline Parameter & Description of Parameter \\
\hline$a_{j}=\rho_{d}^{j} \beta^{j} \rho_{\nu 1}$ & probability a $j$-year old seed develops into an adult plant \\
\hline$s_{j}=\rho_{d}^{j}\left(1-\beta^{j}\right) \rho_{\nu 2}$ & probability a $j$-year old seed becomes a $(j+1)$-year old seed \\
\hline
\end{tabular}

adult population (hence the choice of parameter $a$ ) or the seed bank population (hence the choice of parameter $s$ ). A description of these parameters can be found in Table 2.2.

Note the similar structure of our model (2.1) to the Leslie model (1.6) in Chapter 1. They are both age-structured models with non-negative entries in the first row of the projection matrix and only one possible transition between stages for the remaining rows. There are two major differences between these constructions:

1. Our model allows for density dependent growth and so has nonlinear terms appearing in the growth equations. All terms in the Leslie model are linear.

2. We allow for an infinite number of seed bank cohorts in our model whereas the Leslie model is finite.

\subsection{The Spatial Model}

We next consider how the plant population migrates in space, this is done through seed dispersal. Once an adult plant produces seed, that seed can be spread through a number of methods including wind, active agents or water. The seed eventually sets in the ground and is no longer subject to movement. 
In the context of our model, this dispersal process only affects the new seed population since older seed has already been set in the ground. The reproduction function $F(u) u=H(u)$ describes the production of new seed and hence, we couple a dispersal kernel $k(x)$ with this function. Going back to the flow chart in Figure 2.1, we see that the function $F$ only affects the adult cohort $A_{n}$ and the new seed cohort $S_{n}^{0}$. Thus, we obtain the following integro-difference system to model perennial plants with an age-structured seed bank

$$
\begin{aligned}
A_{n+1}(x) & =\rho_{o} A_{n}(x)+\rho_{d}^{0} \beta^{0} \rho_{\nu 1} \int_{-\infty}^{\infty} k(x-y) H\left(A_{n}(y)\right) d y+\sum_{j=1}^{\infty} \rho_{d}^{j} \beta^{j} \rho_{\nu 1} S_{n}^{j-1}(x) \\
S_{n+1}^{0}(x) & =\rho_{d}^{0}\left(1-\beta^{0}\right) \rho_{\nu 2} \int_{-\infty}^{\infty} k(x-y) H\left(A_{n}(y)\right) d y \\
S_{n+1}^{1}(x) & =\rho_{d}^{1}\left(1-\beta^{1}\right) \rho_{\nu 2} S_{n}^{0}(x) \\
& \vdots \\
S_{n+1}^{m}(x) & =\rho_{d}^{m}\left(1-\beta^{m}\right) \rho_{\nu 2} S_{n}^{m-1}(x)
\end{aligned}
$$

where $k(x)$ is a nonnegative, symmetric, integrable function such that $\int_{-\infty}^{\infty} k(x) d x=$ 1. Using the simplified parameters described in Table 2.2 we have:

$$
\begin{aligned}
A_{n+1}(x) & =\rho_{o} A_{n}(x)+a_{0} \int_{-\infty}^{\infty} k(x-y) H\left(A_{n}(y)\right) d y+\sum_{j=1}^{\infty} a_{j} S_{n}^{j-1}(x) \\
S_{n+1}^{0}(x)= & s_{0} \int_{-\infty}^{\infty} k(x-y) H\left(A_{n}(y)\right) d y \\
S_{n+1}^{1}(x)= & s_{1} S_{n}^{0}(x) \\
& \vdots \\
S_{n+1}^{m}(x) & =s_{m} S_{n}^{m-1}(x)
\end{aligned}
$$


Notice that the integral (i.e. dispersal) only affects the nonlinear components of the model. All terms outside of the integral are linear. 


\section{CHAPTER 3 DIFFERENCE EQUATION ANALYSIS}

In this chapter we consider only the difference equations which model the growth of a perennial plant population with an age-structured seed bank:

$$
\begin{aligned}
A_{n+1}= & \rho_{o} A_{n}+a_{0} H\left(A_{n}\right)+\sum_{j=1}^{\infty} a_{j} S_{n}^{j-1} \\
S_{n+1}^{0}= & s_{0} H\left(A_{n}\right) \\
S_{n+1}^{1}= & s_{1} S_{n}^{0} \\
& \vdots \\
S_{n+1}^{m} & =s_{m} S_{n}^{m-1}
\end{aligned}
$$

Here $A_{n}$ represents the density of the adult plant population at time $n, S_{n}^{j}$ represents the density of the $j$-year old seed bank population at time $n$ and the parameters $a_{j}=\rho_{d}^{j} \beta^{j} \rho_{\nu 1}, s_{j}=\rho_{d}^{j}\left(1-\beta^{j}\right) \rho_{\nu 2}$ are survival and germination rates as described in Table 2.1. This is the system (2.1) which was developed in Chapter 2.

We begin by introducing some notation and definitions. Let

$$
\mathbf{u}=\left(u^{(1)}, u^{(2)}, u^{(3)}, \ldots\right)=\left(A, S^{0}, S^{1}, \ldots\right)
$$

We work in the sequence space $\ell_{1}$ consisting of all real-valued sequences $\mathbf{u}=$ $\left(u^{(1)}, u^{(2)}, u^{(3)}, \ldots\right)$ equipped with norm $\|\cdot\|$ such that

$$
\|\mathbf{u}\|=\sum_{i=1}^{\infty}\left|u^{(i)}\right|
$$


We say $\left\{\mathbf{u}_{n}\right\}$ converges to $\mathbf{u}$ with respect to $\|\cdot\|$ if

$$
\lim _{n \rightarrow \infty}\left\|\mathbf{u}_{n}-\mathbf{u}\right\|=0
$$

where $\mathbf{u}, \mathbf{u}_{n} \in \ell_{1}$ for all $n \in \mathbb{N}$. We denote this convergence as

$$
\left\{\mathbf{u}_{n}\right\} \rightarrow \mathbf{u}
$$

Define the vector-valued operator

$$
\mathbf{P}[\mathbf{u}]:=\left(\begin{array}{c}
\rho_{o} A+a_{0} H(A)+\sum_{j=1}^{\infty} a_{j} S^{j-1} \\
s_{0} H(A) \\
s_{1} S^{0} \\
\vdots \\
s_{m} S^{m-1} \\
\vdots
\end{array}\right)
$$

which is on the right-hand side of (3.1). Thus we have that

$$
\mathbf{u}_{n+1}=\mathbf{P}\left[\mathbf{u}_{n}\right]
$$

We make the following assumptions about the growth function $H$ :

\section{HYPOTHESES 3.1 .}

$i$. There is a positive constant $M$ such that

a. $H(u)$ is continuous for $0 \leq u \leq M$,

b. $H(0)=0$,

c. $0<H(u) \leq M$ for $0<u \leq M$, and

d. $H(u) / u$ is non-increasing for $0<u \leq M$.

ii. There is a positive constant $D \leq M$ such that 
a. $H(u)$ is non-decreasing for $0<u \leq D$,

b. $H(u) / u=F(u)>1$ for $0<u \leq D$, and

c. $H(u) \geq H^{\prime}(0) u-D u^{2}$ for $0<u<D$.

iii. $\beta_{n+1} \leq \beta_{n}$ for all $n \in \mathbb{N}$ and $\lim _{n \rightarrow \infty} \rho_{d}^{n}=0$.

REMARK 3.1. Hypotheses 3.1id, and iib imply that the function $H$ is right differentiable at 0 , that $H^{\prime}(0)>1$, and that $H(u) \leq H^{\prime}(0) u$ for $0 \leq u \leq M$ [22].

The assumptions on the growth function $H$ are common for ecological modcls of this type and, specifically, are realized by both the Ricker function (1.2) and the Beverton-Holt function (1.3). Hypothesis 3.1iii says that germination rates decrease with age and that as seeds become very very old, their survival rates get very close to 0 .

\subsection{Existence of Eigenvalue and Eigenvectors}

The eigenvalue and eigenvectors of a linear system are related to the stability of steady-states. We begin by proving the existence of an eigenvalue and eigenvectors for the difference equation system (3.1) linearized about the origin.

Calculating the Jacobian of the demographic matrix of Eq. (3.1), we find that the linearization about $\mathbf{0}$ is:

$$
\left(\begin{array}{c}
A_{n+1} \\
S_{n+1}^{0} \\
S_{n+1}^{1} \\
\vdots
\end{array}\right)=\left(\begin{array}{cccc}
\rho_{o}+a_{0} H^{\prime}(0) & a_{1} & a_{2} & \ldots \\
s_{0} H^{\prime}(0) & 0 & 0 & \ldots \\
0 & s_{1} & 0 & \ldots \\
0 & 0 & s_{2} & \ldots \\
\vdots & \vdots & \vdots & \vdots
\end{array}\right)\left(\begin{array}{c}
A_{n} \\
S_{n}^{0} \\
S_{n}^{1} \\
\vdots
\end{array}\right)
$$


THEOREM 3.1. Suppose Hypotheses 3.1 hold. Then, there exists a positive eigenvalue $\lambda_{*}$ of $E q$. (3.2) with corresponding strictly positive eigenvectors $\boldsymbol{\xi}=\left(\xi^{(i)}\right)_{i=1}^{\infty} \in$ $\ell_{1}$ given by

$$
\xi^{(j+2)}=\left(\prod_{k=0}^{j} s_{k}\right) \frac{H^{\prime}(0)}{\lambda_{*}^{k+1}} \xi^{(1)}
$$

for all $j \in\{0,1, \ldots\}$ and choice of $\xi^{(1)} \in \mathbb{R}^{+}$.

Proof. To prove the existence of a positive eigenvalue, we show there exists a solution to the following characteristic system corresponding to Eq. (3.2):

$$
\left(\begin{array}{llll}
\rho_{o}+a_{0} H^{\prime}(0) & a_{1} & a_{2} & \ldots \\
s_{0} H^{\prime}(0) & 0 & 0 & \ldots \\
0 & s_{1} & 0 & \ldots \\
\vdots & \vdots & \vdots & \ddots
\end{array}\right)\left(\begin{array}{c}
A \\
S^{0} \\
S^{1} \\
\vdots
\end{array}\right)=\lambda\left(\begin{array}{c}
A \\
S^{0} \\
S^{1} \\
\vdots
\end{array}\right)
$$

Expanding Eq. (3.3) and solving for each seed bank cohort in terms of $A$ gives

$$
\begin{aligned}
A & =\frac{\rho_{o} A+a_{0} H^{\prime}(0) A+a_{1} S^{0}+\ldots+a_{m} S^{m-1}+\ldots}{\lambda} \\
S^{0} & =s_{0}\left(\frac{H^{\prime}(0) A}{\lambda}\right) \\
S^{1} & =\frac{s_{1}}{\lambda} S^{0}=s_{0} s_{1}\left(\frac{H^{\prime}(0) A}{\lambda^{2}}\right) \\
\vdots & \\
S^{m} & =\frac{s_{m}}{\lambda} S^{m-1}=\prod_{i=0}^{m} s_{i}\left(\frac{H^{\prime}(0) A}{\lambda^{m+1}}\right)
\end{aligned}
$$

Substituting into the first equation of (3.4) and dividing by $A$ produces the following infinite series

$$
0=-1+\frac{\rho_{o}}{\lambda}+\frac{a_{0} H^{\prime}(0)}{\lambda}+\frac{a_{1} \Delta_{1} H^{\prime}(0)}{\lambda^{2}}+\ldots+\frac{a_{m} \Delta_{m} H^{\prime}(0)}{\lambda^{m+1}}+\ldots
$$


where $\Delta_{m}=\prod_{i=0}^{m-1} s_{i}$ for all $m \in\{1,2, \ldots\}$.

We show that Eq. (3.5) has a positive solution. To this end, let $\gamma=\frac{1}{\lambda}$ and

$$
G(\gamma)=-1+\rho_{o} \gamma+H^{\prime}(0)\left(a_{0} \gamma+a_{1} \Delta_{1} \gamma^{2}+\ldots+a_{m} \Delta_{m} \gamma^{m+1}+\ldots\right)
$$

Applying the ratio test to the series $\sum_{i=1}^{\infty} a_{i} \Delta_{i} \gamma^{i+1}$ and utilizing Hypothesis 3.1iii we see that $G(\gamma)$ has an infinite radius of convergence.

Observe that $G(0)=-1<0$ and $G\left(\frac{1}{\rho_{o}}\right)>0$. Thus by the Intermediate Value Theorem there exists a solution $\gamma_{*} \in\left[0, \frac{1}{\rho_{o}}\right]$ such that $G\left(\gamma_{*}\right)=0$. Hence Eq. (3.5) has a positive solution, $\lambda_{*}\left(=1 / \gamma_{*}\right)$.

The components of the eigenvectors $\boldsymbol{\xi}=\left(\xi_{i}\right)_{i=1}^{\infty}$ corresponding to $\lambda_{*}$ are given by Eq. (3.4) upon choice of a positive $\xi_{1}$ corresponding to $A$. We note that each $\xi_{i}$ is positive since it is a product of positive parameters.

\subsection{Steady-States}

For a discrete-time system, the steady-states are solutions which are time independent, thus the solutions remain in a steady state. The related steady-state equations for system (3.1) are

$$
\begin{aligned}
& A=\rho_{o} A+a_{0} H(A)+\sum_{j=1}^{\infty} a_{j} S^{j-1} \\
& S^{0}=s_{0} H(A) \\
& S^{1}=s_{1} S^{0} \\
& \vdots \\
& S^{m}=s_{m} S^{m-1}
\end{aligned}
$$




\subsubsection{The Extinction Steady-State}

Clearly $\mathbf{0}=(0,0, \ldots)$ is a steady-state. Biologically this means that if all cohorts of the population have zero density, then they will continue to have zero density for all time, i.e. an extinct population will not spontaneously come into existence. Thus, extinction is a steady-state.

We are interested in the case when the plant population does not go extinct. This is equivalent to evaluating when the steady-state $\mathbf{0}=(0,0, \ldots)$ is unstable. The following theorem gives conditions on the parameters so that we can determine the stability of the extinction equilibrium.

THEOREM 3.2. Suppose Hypotheses 3.1 hold. Then the steady-state $0=(0,0, \ldots)$ of system (3.1) is unstable if

$$
1<\rho_{o}+H^{\prime}(0)\left(a_{0}+a_{1} \Delta_{1}+\ldots+a_{m} \Delta_{m}+\ldots\right)
$$

and stable if the inequality is reversed. Here, $\Delta_{m}=\prod_{i=0}^{m-1} s_{i}$ for all $m \in \mathbb{N}$.

Proof. To show that the extinction steady-state is unstable, we show that, under condition (3.7), $\lambda_{*}>1$ in the characteristic equation linearized about 0.

Following the procedure in the proof of Theorem 3.1 we see that the characteristic polynomial is

$$
G(\gamma)=-1+\rho_{o} \gamma+H^{\prime}(0)\left(a_{0} \gamma+a_{1} \Delta_{1} \gamma^{2}+\ldots+a_{m} \Delta_{m} \gamma^{m+1}+\ldots\right)
$$

We show there exists $0<\gamma^{*} \leq 1$ such that $G\left(\gamma^{*}\right)=0$.

Observe that $G(0)=-1<0$. Also, $G(1)>0$ by condition (3.7) and $G(1)<\infty$ since $G$ has an infinite radius of convergence.

Therefore, by the Intermediate Value Theorem, there exists a solution $0<$ $\gamma^{*} \leq 1$ such that $G\left(\gamma^{*}\right)=0$. Hence $\lambda_{*}>1$ (i.e $\mathbf{0}$ is unstable) when condition (3.7) holds. 
Alternatively, we show that when condition (3.7) is reversed then $\lambda_{*}<1$ (i.e. 0 is stable). To this end, note that $G(1)<0$ by condition (3.7). Also, $G(\infty)=\infty$ and since $G$ is continuous there must exist $d \in(1, \infty)$ such that $G(d)>0$. Thus, by the Intermediate Value Theorem, there exists a solution $1<\gamma^{*}<d$ such that $G\left(\gamma^{*}\right)=0$. Hence there exists $\lambda_{*}<1$ when condition (3.7) is reversed.

The following theorem states that the population will go extinct if the origin 0 is stable.

THEOREM 3.3. Suppose Hypotheses 3.1 hold and the extinction steady-state $\mathbf{0}=$ $(0,0, \ldots)$ is stable (i.e. $\left.\lambda_{*}<1\right)$. Then solutions to Eq. (3.1) converge to 0 if $\mathbf{u}_{0} \leq \boldsymbol{\xi}$

Proof. By Remark 4.1, $H(u) \leq H^{\prime}(0) u$, and so $\mathbf{P}[\mathbf{u}] \leq \mathbf{L}[\mathbf{u}]$ where $\mathbf{L}$ is the linearization of $\mathbf{P}$ at $\mathbf{0}$. Thus,

$$
\mathbf{u}_{1}=\mathbf{P}\left[\mathbf{u}_{0}\right] \leq \mathbf{L}\left[\mathbf{u}_{0}\right] \leq \mathbf{L}[\boldsymbol{\xi}]=\lambda_{*} \boldsymbol{\xi}
$$

Therefore, by induction,

$$
\mathbf{u}_{n} \leq \lambda_{*}^{n} \boldsymbol{\xi}
$$

Taking the limit of Eq. (3.8) as $n \rightarrow \infty$ we have $\left\{\mathbf{u}_{n}\right\} \rightarrow \mathbf{0}$ since $\lambda_{*}<1$.

\subsubsection{Existence of a Positive Steady-State}

We are now turn our attention to circumstances under which the population has a survival steady-state or a positive steady-state. The following theorem states that there exists a positive steady-state to the recursion (3.1) whenever the extinction steady-state $\mathbf{0}=(0,0, \ldots)$ is unstable.

THEOREM 3.4. Suppose Hypotheses 3.1 hold. Then there exists a strictly positive, unique solution $\mathbf{u}_{*}=\left(A_{*}, S_{*}^{0}, S_{*}^{1}, \ldots\right)$ to the steady-state equation (3.6) if condition (3.7) is satisfied. Further, $\mathbf{u}_{*} \in \ell_{1}$ (i.e. $\left.\left\|\mathbf{u}_{*}\right\|<\infty\right)$. 
Proof. For every $m$, solving for $S^{m}$ in terms of $A$ in system (3.6) and substituting those values into the adult population equation gives

$$
\begin{aligned}
A & =\rho_{o} A+a_{0} H(A)+a_{1} s_{0} H(A)+a_{2} s_{0} s_{1} H(A)+\ldots \\
& =\rho_{o} A+a_{0} H(A)+\sum_{j=1}^{\infty}\left[a_{j} \Delta_{j} H(A)\right]
\end{aligned}
$$

where $\Delta_{j}=\prod_{i=0}^{j-1} s_{i}$ for all $j \in\{1,2, \ldots\}$.

Dividing by $A$ and solving for $\frac{H(A)}{A}$ we have

$$
\frac{H(A)}{A}=\frac{1-\rho_{o}}{a_{0}+\sum_{j=1}^{\infty} a_{j} \Delta_{j}} .
$$

Recall that $s_{i}=\rho_{d}^{i}\left(1-\beta^{i}\right) \rho_{\nu 2}$ and so for each $i, \Delta_{i}$ has a factor of $\left(\rho_{\nu 2}\right)^{i}$. Hence, the denominator of the right-hand side of (3.9) converges because it is bounded above by the geometric sequence $a_{0}+\left\{\left(\rho_{\nu 2}\right)^{i}\right\}_{i=1}^{\infty}$. Let the constant $B=\frac{1-\rho_{o}}{a_{0}+\sum_{j=1}^{\infty} a_{j} \Delta_{j}}$ and

$$
G(A)=\frac{H(A)}{\Lambda}-B
$$

We will show there exists solution $A_{*}$ to Eq. (3.9) by applying the Intermediate Value Theorem to $G(A)$.

By Hypotheses 3.1, $H(A)$ is bounded above by $M$. Hence,

$$
\begin{aligned}
G(\infty) & =\lim _{A \rightarrow \infty} \frac{H(A)}{A}-B \\
& \leq \lim _{A \rightarrow \infty} \frac{M}{A}-B \\
& =-B \\
& <0 .
\end{aligned}
$$

Also, by the condition (3.7), $H^{\prime}(0)-B>0$. Thus,

$$
\begin{aligned}
G(0) & =\lim _{A \rightarrow 0} \frac{H(A)}{A}-B \\
& =H^{\prime}(0)-B \\
& >0 .
\end{aligned}
$$


Therefore, by the Intermediate Value Theorem, there exists $0<A_{*}<\infty$ such that $G\left(A_{*}\right)=0$. Hence there exists a positive steady-state $\mathbf{u}_{*}=\left(A_{*}, S_{*}^{0}, S_{*}^{1}, \ldots\right)$. Furthermore, $G(A)$ is monotone since $\frac{H(A)}{A}$ is monotone (Hypotheses 3.1 ) and so this steady-state must be unique.

To show $\left\|\mathbf{u}_{*}\right\|<\infty$ observe that for $i=0,1, \ldots$,

$$
\begin{aligned}
S_{*}^{(i)} & =H\left(A_{*}\right) \Delta_{i-1} \\
& <H\left(A_{*}\right)\left(\rho_{\nu 2}\right)^{i-1}
\end{aligned}
$$

Thus,

$$
\begin{aligned}
\left\|\mathbf{u}_{*}\right\| & =\Lambda_{*}+\sum_{i=0}^{\infty} S_{*}^{(i)} \\
& <A_{*}+H\left(A_{*}\right) \sum_{i=1}^{\infty}\left(\rho_{\nu 2}\right)^{i} \\
& <\infty
\end{aligned}
$$

Therefore, $\mathbf{u}_{*} \in \ell_{1}$ and the theorem is proved.

REMARK 3.2. There does not exist a steady-state of system (3.1) which has both positive and zero components. Thus, the only steady-states of system (3.1) are $\mathbf{0}$ and $\mathbf{u}_{*}$.

The remark is easy to see for if there is one component of a steady-state which is zero, it will force all other components to be zero as well.

\subsubsection{Convergence to the Positive Steady-State}

We now know when a positive steady-state exists, but we do not know how solutions behave around the positive steady-state. What is the stability of the positive steady-state? Does the population eventually converge to the steadystate? What information about the population can we extract knowing that a positive steady-state exists? 
The following theorem says that if we have an initial population that is less than the positive steady-state and the growth function is non-decreasing, then the positive steady-state is stable. Thus populations (that are not initially too big) whose growth increases with density will survive and increase to a stable population over time.

THEOREM 3.5. Suppose Hypotheses 3.1 hold, the reproduction function $H(A)$ is non-decreasing and the steady state $\mathbf{0}$ is unstable. Then solutions to system (3.1) converge to the positive steady state $\mathbf{u}_{*}=\left(A_{*}, S_{*}^{0}, S_{*}^{1}, \ldots\right)$ with respect to $\|\cdot\|$ when

$$
\mathbf{0}<\mathbf{u}_{0} \leq \mathbf{u}_{*}, \mathbf{u}_{0} \not \equiv \mathbf{0}
$$

Proof. Let $\xi=\left(\xi^{(1)}, \xi^{(2)}, \ldots\right)$ be a positive eigenvector corresponding to the positive eigenvalue $\lambda_{*}$ of the linearized system (3.3).

Since $H(A)$ is non-decreasing and all other terms in system (3.1) are linear, then we have that $\mathbf{P}$ is order preserving (i.e. $\mathbf{u} \geq \mathbf{v} \Longrightarrow \mathbf{P}[\mathbf{u}] \geq \mathbf{P}[\mathbf{v}]$ ).

We first show that the theorem holds for the simpler case when the initial population is bounded below by an eigenvector.

LEMMA 3.1. Suppose the hypotheses of Theorem 3.5 hold and there exists a sufficiently small positive constant $\eta$ such that

$$
\eta \boldsymbol{\xi} \leq \mathbf{u}_{0} \leq \mathbf{u}_{*}
$$

Then $\left\{\mathbf{u}_{n}\right\} \rightarrow \mathbf{u}_{*}$.

Proof. First, suppose $\overline{\mathbf{u}}_{0}=\eta \boldsymbol{\xi}$. We proceed to show by induction that $\overline{\mathbf{u}}_{n+1} \geq \overrightarrow{\mathbf{u}}_{n}$. Since $\lambda_{*}>1$, it is easy to show that the linear terms of $\overline{\mathbf{u}}_{n}$ are nondecreasing. To show that the nonlinear terms $\left(u_{n}^{(1)}, u_{n}^{(2)}\right)$ are non-decreasing recall that $H(u) \geq H^{\prime}(0) u-D u^{2}$ by Hypothesis 3.1. We may choose $\eta$ sufficiently small so that $D \eta \max \left\{\xi^{(1)}: \frac{\left(\xi^{(1)}\right)^{2}}{\xi^{(2)}}\right\}<\lambda_{*}-1$. Using this fact and the fact that $\lambda_{*}>1$ 
we obtain the following inequalities:

$$
\begin{aligned}
& \overline{\mathbf{u}}_{1}=\mathbf{P}\left[\overline{\mathbf{u}}_{0}\right]=\mathbf{L}\left[\overline{\mathbf{u}}_{0}\right]+\mathbf{P}\left[\overline{\mathbf{u}}_{0}\right]-\mathbf{L}\left[\overline{\mathbf{u}}_{0}\right] \\
& =\mathbf{L}\left[\overline{\mathbf{u}}_{0}\right]+\left(\begin{array}{c}
a_{0}\left(H\left(u_{0}^{(1)}\right)-H^{\prime}(0) u_{0}^{(1)}\right) \\
s_{0}\left(H\left(u_{0}^{(1)}\right)-H^{\prime}(0) u_{0}^{(1)}\right) \\
0 \\
\vdots
\end{array}\right) \\
& \geq \mathrm{L}\left[\overline{\mathbf{u}}_{0}\right]-\left(\begin{array}{c}
a_{0} D\left(u_{0}^{(1)}\right)^{2} \\
s_{0} D\left(u_{0}^{(1)}\right)^{2} \\
0 \\
\vdots
\end{array}\right) \\
& =\eta \lambda_{*} \boldsymbol{\xi}-\left(\begin{array}{c}
a_{0} D\left(\eta \xi^{(1)}\right)^{2} \\
s_{0} D\left(\eta \xi^{(1)}\right)^{2} \\
0 \\
\vdots
\end{array}\right) \\
& =\left(\begin{array}{c}
\eta \xi^{(1)}\left(\lambda_{*}-a_{0} D \eta \xi^{(1)}\right) \\
\eta \xi^{(2)}\left(\lambda_{*}-s_{0} D \eta \frac{\left(\xi^{(1)}\right)^{2}}{\xi^{(2)}}\right) \\
\eta \xi^{(3)} \lambda_{*} \\
\vdots
\end{array}\right) \\
& >>\eta \boldsymbol{\xi} \\
& =\overrightarrow{\mathbf{u}}_{0} .
\end{aligned}
$$

Since $\mathbf{P}$ is order preserving, we conclude by induction that

$$
\overrightarrow{\mathbf{u}}_{n+1}>>\overline{\mathbf{u}}_{n}
$$

Similarly, since $\overline{\mathbf{u}}_{0} \leq \mathbf{u}_{*}$, by induction we have that $\overline{\mathbf{u}}_{n} \leq \mathbf{u}_{*}$. Thus, $\left\{\overline{\mathbf{u}}_{n}\right\}$ is increasing and bounded above by $\mathbf{u}_{*}$, therefore

$$
\left\{\overrightarrow{\mathbf{u}}_{n}\right\} \rightarrow \mathbf{u}_{*}
$$


Now we use the fact that $\overline{\mathbf{u}}_{0}=\eta \boldsymbol{\xi} \leq \mathbf{u}_{0} \leq \mathbf{u}_{*}$ and conclude by induction that

$$
\overline{\mathbf{u}}_{n} \leq \mathbf{u}_{n} \leq \mathbf{u}_{*}
$$

Taking the limit as $n \rightarrow \infty$ of Eq. (3.11), gives $\mathbf{u}_{*} \leq \mathbf{u}_{n} \leq \mathbf{u}_{*}$. Therefore, the lemma is satisfied by the Squeeze Theorem.

We proceed to prove the theorem for the case when some of the initial population densities may be 0 . The idea is that we can choose a generation far enough in the future so that the first $N$ cohorts have positive population densities and will therefore converge to the positive steady state by Lemma 3.1. The infinite number of remaining cohorts are so small that their norm can be tempered by any $\epsilon$.

To this end, let $t>0$. Since $\left\|\mathbf{u}_{*}\right\|<\infty$ we may choose $N \in \mathbb{N}$ such that

$$
\sum_{i=N+1}^{\infty} u_{*}^{(i)}<\frac{\epsilon}{3}
$$

Using the fact that $\mathbf{P}$ is order preserving and $\mathbf{u}_{0} \leq \mathbf{u}_{*}$, we conclude by induction that $\mathbf{u}_{n} \leq \mathbf{u}_{*}$ for all $n \in \mathbb{N}$. Thus, $\left\|\mathbf{u}_{n}\right\|<\left\|\mathbf{u}_{*}\right\|<\infty$.

We may now choose $M \in \mathbb{N}$ such that

$$
\sum_{i=N+1}^{\infty} u_{M}^{(i)}<\frac{\epsilon}{3}
$$

and

$$
u_{M}^{(i)}>0 \text { for all } i \in\{1, \ldots, N\} .
$$

Thus,

$$
\begin{aligned}
\sum_{i=N+1}^{\infty}\left|u_{M}^{(i)}-u_{*}^{(i)}\right| & \leq \sum_{i=N+1}^{\infty}\left|u_{M}^{(i)}\right|+\sum_{i=N+1}^{\infty}\left|u_{*}^{(i)}\right| \\
& <\frac{2 \epsilon}{3}
\end{aligned}
$$


Now, since $u_{M}^{(i)}>0$ for all $i \in\{1, \ldots, N\}$, we may choose $\eta$ sufficiently small such that

$$
\left(\begin{array}{c}
u_{M}^{(1)} \\
\vdots \\
u_{M}^{(N)}
\end{array}\right)>\eta\left(\begin{array}{c}
\xi^{(1)} \\
\vdots \\
\xi^{(N)}
\end{array}\right)
$$

By a truncated version of Lemma 3.1 we conclude that

$$
\lim _{M \rightarrow \infty}\left(\begin{array}{c}
u_{M}^{(1)} \\
\vdots \\
u_{M}^{(N)}
\end{array}\right)=\left(\begin{array}{c}
u_{*}^{(1)} \\
\vdots \\
u_{*}^{(N)}
\end{array}\right)
$$

Now we may choose $M_{i} \in \mathbb{N}$ such that for every $m_{i}>M_{i}$

$$
\left(\begin{array}{c}
u_{m_{1}}^{(1)}-u_{*}^{(1)} \\
\vdots \\
u_{m_{N}}^{(N)}-u_{*}^{(N)}
\end{array}\right)<\left(\begin{array}{c}
\frac{\epsilon}{3 N} \\
\vdots \\
\frac{\epsilon}{3 N}
\end{array}\right)
$$

Let $M_{M}=\max \left\{M_{i}\right\}$. Then,

$$
\sum_{i=1}^{N}\left|u_{M_{M}}^{(i)}-u_{*}^{(i)}\right|<\frac{\epsilon}{3} .
$$

Finally, letting $M_{*}=\max \left\{M, M_{M}\right\}$ we have by Eq. (3.12) and (3.13) that,

$$
\begin{aligned}
\left\|\mathbf{u}_{M_{*}}-\mathbf{u}_{*}\right\| & =\sum_{i=1}^{\infty}\left|u_{M_{*}}^{(i)}-u_{*}^{(i)}\right| \\
& =\sum_{i=1}^{N}\left|u_{M_{*}}^{(i)}-u_{*}^{(i)}\right|+\sum_{i=N+1}^{\infty}\left|u_{M_{*}}^{(i)}-u_{*}^{(i)}\right| \\
& <\frac{\epsilon}{3}+\frac{2 \epsilon}{3} \\
& =\epsilon .
\end{aligned}
$$

Therefore, $\left\{\mathbf{u}_{n}\right\} \rightarrow \mathbf{u}_{*}$ with respect to $\|\cdot\|$ and the theorem is proved. 


\subsubsection{Connection to Analogous Finite System}

Consider the $m$-dimensional truncated version of system (3.1), meaning that no seed can reach an age greater than $m-2$-years old. We append an index to the subscript of each cohort to denote this truncation (i.e. $A_{n \mid m}$ represents the $n$-th generation of the adult plant population for the $m$-dimensional finite system). The system of equations now become:

$$
\begin{aligned}
A_{(n+1) \mid m} & =\rho_{o} A_{n \mid m}+a_{0} H\left(A_{n \mid m}\right)+\sum_{j=1}^{m-1} a_{j} S_{n \mid m}^{j-1} \\
S_{(n+1) \mid m}^{0} & =s_{0} H\left(A_{n \mid m}\right) \\
S_{(n+1) \mid m}^{1} & =s_{1} S_{n \mid m}^{0} \\
& \vdots \\
S_{(n+1) \mid m}^{m-2} & =s_{m-2} S_{n \mid m}^{m-3} .
\end{aligned}
$$

THEOREM 3.6. Suppose Hypotheses 3.1 hold. Let $\mathbf{u}_{*}=\left(A_{*}, S_{*}^{0}, S_{*}^{1}, \ldots\right)$ be the positive steady-state associated with system (3.1) and let $\mathbf{u}_{* \mid m}=\left(A_{* \mid m}, S_{* \mid m}^{0}, \ldots, S_{* \mid m}^{m}\right)$ be the positive steady-state associated with the m-dimensional truncated system (3.14). Then,

$$
\lim _{m \rightarrow \infty} \mathbf{u}_{* \mid m}=\mathbf{u}_{*}
$$

Proof. Recall that Eq. (3.9) is the steady-state characteristic equation corresponding to system (3.1) and so

$$
\frac{H\left(A_{*}\right)}{A_{*}}=\frac{1-\rho_{o}}{a_{0}+\sum_{j=1}^{\infty} a_{j} \Delta_{j}} .
$$

where $\Delta_{j}=\prod_{i=0}^{j-1} s_{i}$ for all $j \in\{1,2, \ldots\}$.

Similarly, for the truncated system we have that

$$
\frac{H\left(A_{* \mid m}\right)}{A_{* \mid m}}=\frac{1-\rho_{o}}{a_{0}+\sum_{j=1}^{m-1} a_{j} \Delta_{j}} .
$$

Clearly $\frac{H\left(A_{* \mid(m+1)}\right)}{A_{* \mid(m+1)}}<\frac{H\left(A_{* \mid m}\right)}{A_{* \mid m}}$ and $\frac{H\left(A_{*}\right)}{A_{*}}<\frac{H\left(A_{* \mid m}\right)}{A_{* \mid m}}$ for all $m \in \mathbb{N}$ since $a_{j}, \Delta_{j}>0$ for all $j$. Hence, since $\frac{H(A)}{A}$ is non-increasing and continuous then 
1. $A_{* \mid(m+1)}<A_{* \mid m}$ for all $m \in \mathbb{N}$

2. $A_{*}<A_{* i m}$ for all $m \in \mathbb{N}$.

Thus $\left\{A_{* \mid m}\right\}_{m=1}^{\infty}$ is a decreasing sequence that is bounded below by $A_{*}$ and so $\lim _{m \rightarrow \infty} A_{* \mid m}=A_{*}$.

Similarly we may conclude that $\lim _{m \rightarrow \infty} S_{* \mid m}^{j}=S_{*}^{j}$ for all $j \in \mathbb{N}$ and the theorem is proved.

\subsection{Solution Curves and Bifurcation Diagrams}

In this section we present solution curves and bifurcation diagrams of the 4dimensional finite difference equation model (3.14) with the Ricker growth function

$$
H(A)=A e^{r-A}
$$

with $r>0$.

All figures use the following color scheme:

- Black line - $A$, adult plant population

- Blue line - $S^{0}$, new seed population

- Green line - $S^{1}, 1$-year old seed population

- Red line - $S^{2}, 2$-year old seed population

Figure 3.1 displays solution curves for 4 different values of $r$ when $\rho_{o}=0.25$,

$\rho_{d}^{0}=\rho_{d}^{1}=\rho_{d}^{2}=\rho_{d}^{3}=1, \rho_{\nu 1}=\rho_{\nu 2}=0.9, \beta^{0}=0.7, \beta^{1}=0.5, \beta^{2}=0.3, \beta^{3}=0.1$.

Figure 3.1a shows that when $r=1$ the populations are stable. As $r$ increases, the populations are eventually periodic; when $r=3.5$ (Figure $3.1 \mathrm{~b}$ ) solutions have period 2 and when $r=4.5$ (Figure 3.1c) solutions have period 4. Finally, when $r$ is large enough (in Figure 3.1d, $r=5.5$ ), solutions are chaotic. 


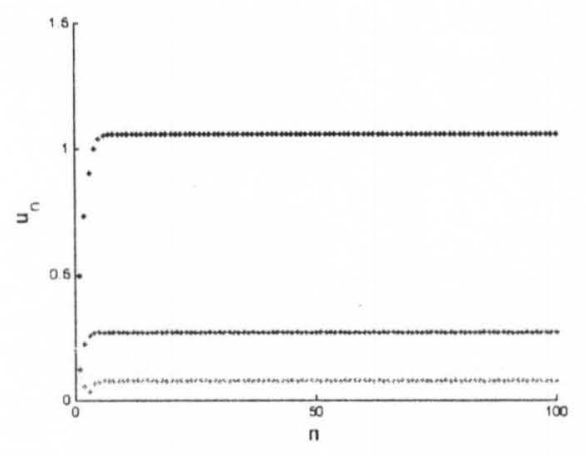

(a) $r=1$, stable solutions

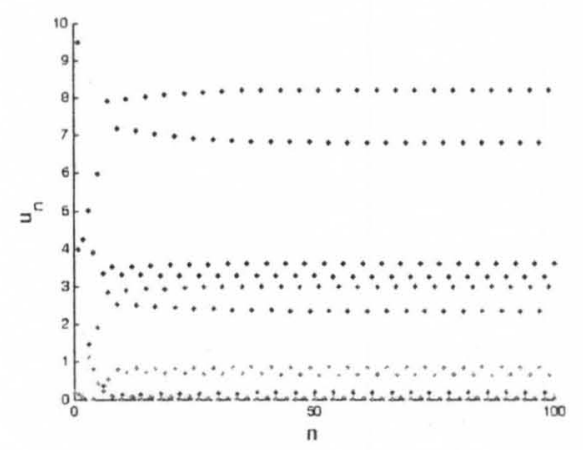

(c) $r=4.5$, period 4 oscillations

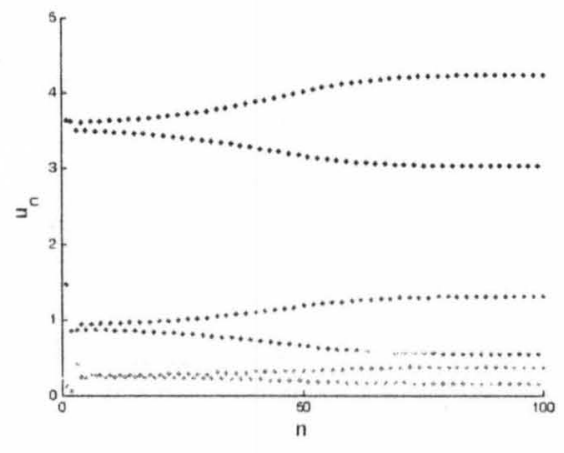

(b) $r=3.5$, period 2 oscillations

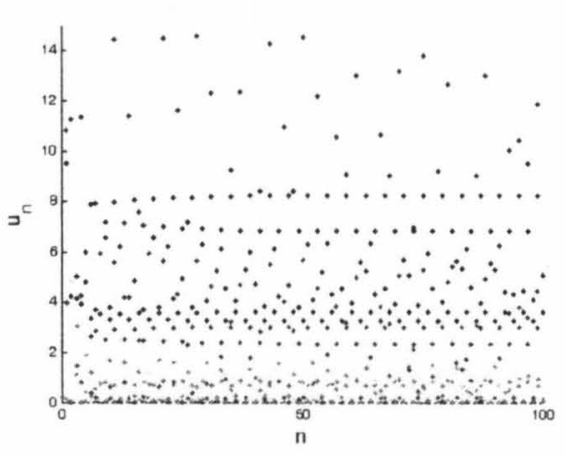

(d) $r=5.5$, chaos

FIGURE 3.1-Solution curves of (3.1) with $H$ the Ricker function.

Figure 3.2a displays a bifurcation diagram with bifurcation parameter $r$, depicting the long term dynamics of the solution curves in Figure 3.1. All four population cohorts are reminiscent of the logistic bifurcation which goes through some period doubling then moves to chaos (as shown in the solution curves). Each population cohort appear to have stability changes at concurrent $r$ values. For example, the first bifurcation point in each diagram appears to occur around the value $r=3.5$.

Figure $3.2 \mathrm{~b}$ plots the adult bifurcation when there is a seed bank present (black) and when there is no seed bank present (magenta). The diagrams use the same parameters as those defined above except for the case of no seed bank we 


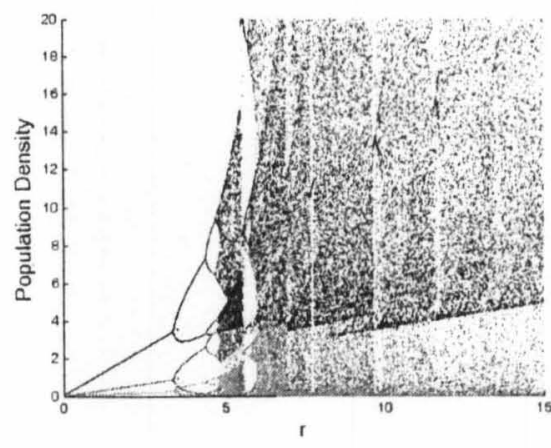

(a) Seed bank cohorts

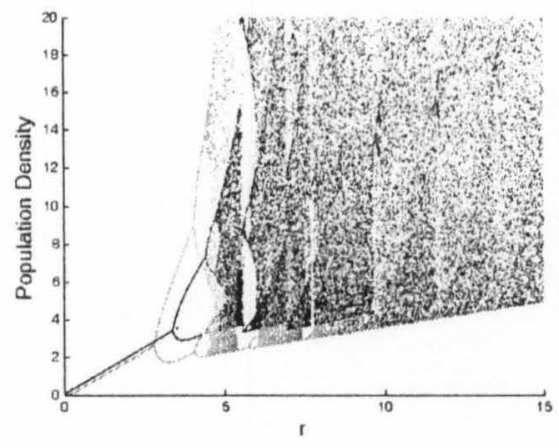

(b) Seed bank vs. no seed bank

FIGURE 3.2 - The bifurcation diagrams of a truncated version of (3.1) with $H$ the Ricker function and $r$ the bifurcation parameter, $r \in[0,15]$.

have that $s_{i}=0$. We see here that the seed bank can have a stabilizing effect on the population dynamics in that the period doubling occurs later in the seed bank diagram.

Figure 3.3 uses the new seed germination rate $\beta^{0}$ as the bifurcation parameter and $\rho_{o}=0.5, \rho_{d}^{0}=\rho_{d}^{1}=\rho_{d}^{2}=\rho_{d}^{3}=1, \rho_{\nu 1}=\rho_{\nu 2}=0.9, \beta^{1}=0.1, \beta^{2}=0.05$, $\beta^{3}=0.025$. We see from Figures $3.3 \mathrm{a}$ and $3.3 \mathrm{~b}$ that when $r=1$ or 3 the population dynamics are stable regardless of the value of $\beta^{0}$. However, when $r=5$ or 7 we see some interesting changes. Figures $3.3 \mathrm{c}$ and $3.3 \mathrm{~d}$ show that when $\beta^{0}$ is small enough, solutions are chaotic but as $\beta^{0}$ increases, the population stabilizes, moves into period doubling and then gets chaotic again as $\beta^{0}$ nears 1 . This pattern of chaos to stability and back occurs often in this model, in fact, every set of parameter choices we used produced similar looking bifurcations. To the best of our knowledge, this type of switching behavior has not been observed in single species models before and thus opens up new areas for speculation. We have not been able to postulate a clear biological interpretation for this behavior. These bifurcations give more questions than answers but are worthy of further investigation. 


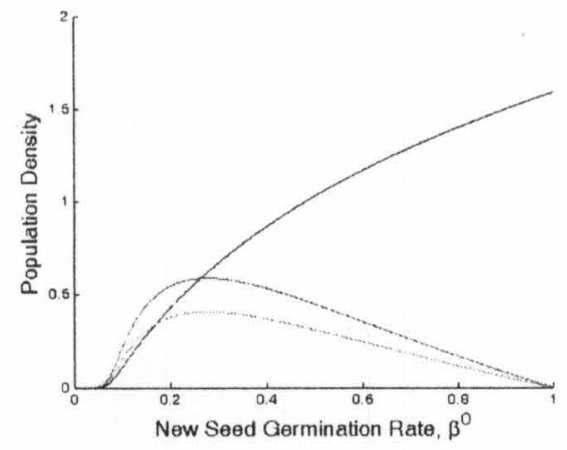

(a) $r=1$

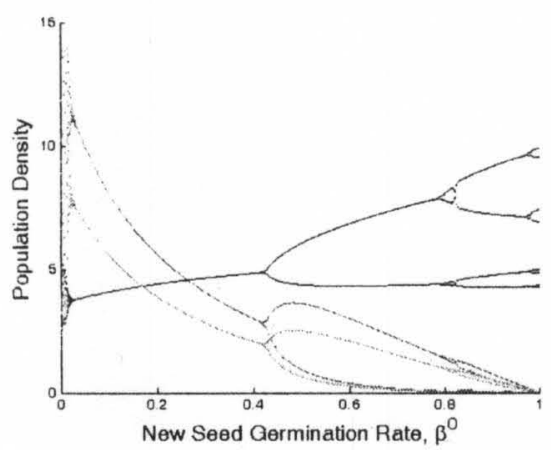

(c) $r=5$

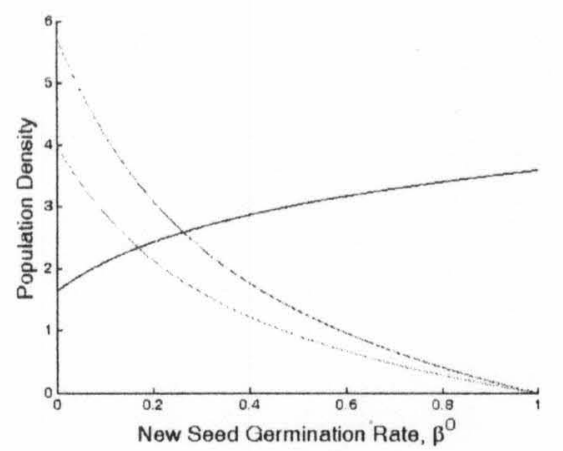

(b) $r=3$

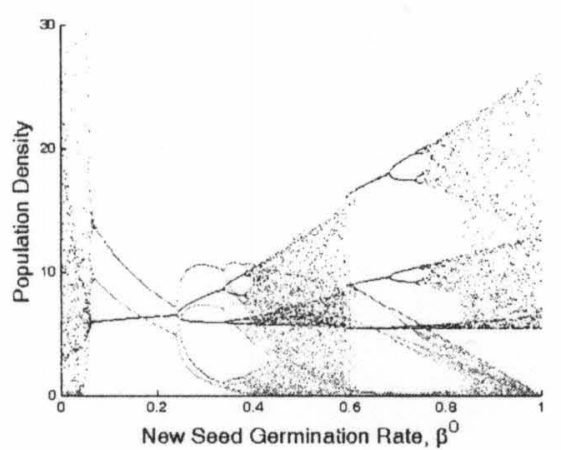

(d) $r=7$

FIGURE 3.3-The bifurcation diagrams of a truncated version of (3.1) with $H$ the Ricker function and the new seed germination rate $\beta_{0}$ as the bifurcation parameter, $\beta^{0} \in[0,1]$. 


\section{CHAPTER 4 SPATIAL ANALYSIS}

In this chapter we analyze the spatial system which models spread of perennial plant populations with an age-structured seed bank:

$$
\begin{aligned}
A_{n+1}(x) & =\rho_{o} A_{n}(x)+a_{0} \int_{-\infty}^{\infty} k(x-y) H\left(A_{n}(y)\right) d y+\sum_{j=1}^{\infty} a_{j} S_{n}^{j-1}(x) \\
S_{n+1}^{0}(x) & =s_{0} \int_{-\infty}^{\infty} k(x-y) H\left(A_{n}(y)\right) d y \\
S_{n+1}^{1}(x)= & s_{1} S_{n}^{0}(x) \\
& \vdots \\
S_{n+1}^{m}(x) & =s_{m} S_{n}^{m-1}(x)
\end{aligned}
$$

Here $A_{n}(x)$ represents the density distribution of the adult plant population at time $n, S_{n}^{j}(x)$ represents the density distribution of the $j$-year old seed bank population at time $n$ and the parameters $a_{j}=\rho_{d}^{j} \beta^{j} \rho_{\nu 1}, s_{j}=\rho_{d}^{j}\left(1-\beta^{j}\right) \rho_{\nu 2}$ represent survival and germination probabilities as described in Tables 2.1 and 2.2. This is the system (2.4) which was developed in Chapter 2. 
Define the vector-valued operator

$$
\mathbf{Q}[\mathbf{u}](x):=\left(\begin{array}{c}
\rho_{o} A(x)+a_{0} \int_{-\infty}^{\infty} k(x-y) H(A(y)) d y+\sum_{j=1}^{\infty} a_{j} S^{j-1}(x) \\
s_{0} \int_{-\infty}^{\infty} k(x-y) H(A(y)) d y \\
s_{1} S^{\prime 0}(x) \\
\vdots \\
s_{m} S^{m-1}(x) \\
\vdots
\end{array}\right)
$$

which is on the right-hand side of (4.1). Thus we have that

$$
\mathbf{u}_{n+1}(x)=\mathrm{Q}\left[\mathbf{u}_{n}\right](x)
$$

where $\mathbf{u}(x)=\left(u^{(1)}(x), u^{(2)}(x), \ldots\right)=\left(A(x), S^{0}(x), \ldots\right)$.

We extend the sequence space $\ell_{1}$ used in Chapter 3 to an infinite-dimensional vector space consisting of all real-valued continuous function sequences $\mathbf{u}(x)=$ $\left(u^{(1)}(x), u^{(2)}(x), \ldots\right)$.

When we discuss convergence of a sequence of infinite-dimensional vectorvalued functions $\left\{\mathbf{u}_{n}(x)\right\}_{n=1}^{\infty}$ we are referring to component-wise convergence. We say $\left\{\mathbf{u}_{n}(x)\right\}$ converges to $\mathbf{u}(x)$ with respect to the supremum norm $\|\cdot\|$ if

$$
\lim _{n \rightarrow \infty}\left\|u_{n}^{(i)}(x)-u^{(i)}(x)\right\|=0
$$

for every $i=1,2, \ldots$ where

$$
\|u(x)\|=\sup \{|u(x)|: x \in \mathbb{R}\}
$$

We will sometimes refer to the $m$-dimensional finite analog of system (4.1) 
which we distinguish by using an additional subscript next to the time subscript:

$$
\begin{aligned}
A_{n+1 \mid m}(x) & =\rho_{o} A_{n \mid m}(x)+a_{0} \int_{-\infty}^{\infty} k(x-y) H\left(A_{n \mid m}(y)\right) d y+\sum_{j=1}^{n-1} a_{j} S_{n \mid m}^{j-1}(x) \\
S_{n+1 \mid m}^{0}(x) & =s_{0} \int_{-\infty}^{\infty} k(x-y) H\left(A_{n \mid m}(y)\right) d y \\
S_{n+1 \mid m}^{1}(x) & =s_{1} S_{n \mid m}^{0}(x) \\
& \vdots \\
S_{n+1 \mid m-2}^{m-2}(x) & =s_{m} S_{n \mid m}^{m-3}(x)
\end{aligned}
$$

where $m \in \mathbb{N}$. Similarly we define the corresponding $m$-dimensional operator $\mathrm{Q}_{m}[\mathbf{u}]$ so that:

$$
\mathbf{u}_{n+1 \mid m}=\mathbf{Q}_{m}\left[\mathbf{u}_{n \mid m}\right](x)
$$

We make the following assumptions throughout this chapter.

\section{HYPOTHESES 4.1.}

i. There is a positive constant $\kappa_{0}$ such that

a. $H(u)$ is continuous for $0 \leq u \leq \kappa_{0}$,

b. $H(0)=0$,

c. $0<H(u) \leq \kappa_{0}$ for $0<u \leq \kappa_{0}$, and

d. $H(u) / u$ is non-increasing for $0<u \leq \kappa_{0}$.

ii. There is a positive constant $d \leq \kappa_{0}$ such that

a. $H(u)$ is non-decreasing for $0<u \leq d$,

b. $H(u) / u=F(u)>1$ for $0<u<d$, and

c. $H(u) \geq H^{\prime}(0) u-d u^{2}$ for $0<u<d$.

iii. $\beta_{n+1} \leq \beta_{n}$ for all $n \in \mathbb{N}$ and $\lim _{n \rightarrow \infty} \rho_{d}^{n}=0$. 
iv. $1<\rho_{o}+H^{\prime}(0)\left[a_{0}+a_{1} s_{0}+a_{2} s_{0} s_{1}+\ldots+a_{m} \prod_{i=1}^{m-1} s_{i}+\ldots\right]$.

$v . k(x)$ is a continuous, symmetric, non-negative function such that

a. $\int_{\mathbb{R}} k(x) d x=1$, and

b. the integral

$$
K(\mu):=\int_{\mathbb{R}} k(x) e^{\mu x} d x
$$

converges for all real number $\mu$ and $K(\mu)>1$.

REMARK 4.1. Hypotheses 4.1id, and iib imply that the function $H$ is right differentiable at 0 , that $H^{\prime}(0)>1$, and that $H(u) \leq H^{\prime}(0) u$ for $0 \leq u \leq \kappa_{0}$ [22].

The assumptions on the growth function $H$ and model parameters ( $\mathrm{Hy}-$ potheses 4.1i, ii, iii) are identical to Hypotheses 3.1 in Chapter 3. We note again that these first two are common for ecological models of this type and, specifically, are realized by both the Ricker function (1.2) and the Beverton-Holt function (1.3).

Note that Hypothesis $4.1 \mathrm{iv}$ is the Condition 3.7 from Chapter 3. By Theorem 3.2, this condition implies that the extinction steady-state $\mathbf{0}$ of the difference equation system is unstable (i.e. solutions move away from the origin).

Now define the linearized moment generating matrix $\mathbf{B}_{\mu}$ as

$$
\mathbf{B}_{\mu}:=\left(\begin{array}{cccc}
\rho_{0}+a_{0} H^{\prime}(0) K(\mu) & a_{1} & a_{2} & \ldots \\
s_{0} H^{\prime}(0) K(\mu) & 0 & 0 & \ldots \\
0 & s_{1} & 0 & \ldots \\
0 & 0 & s_{2} & \ldots \\
\vdots & \vdots & \vdots & \ddots
\end{array}\right)
$$

where $K(\mu)=\int_{\mathbb{R}} k(x) e^{\mu x} d x$ as defined in Hypothesis 4.1vb. Define the $m$-dimensional 
analog of this matrix as

$$
\mathbf{B}_{\mu \mid m}:=\left(\begin{array}{ccccc}
\rho_{o}+a_{0} H^{\prime}(0) K(\mu) & a_{1} & \ldots & a_{m-2} & a_{m-1} \\
s_{0} H^{\prime}(0) K(\mu) & 0 & \ldots & 0 & 0 \\
0 & s_{1} & \ldots & 0 & 0 \\
\vdots & \vdots & \ddots & \vdots & \vdots \\
0 & 0 & \ldots & s_{m-2} & 0
\end{array}\right) .
$$

We present a lemma about the finite spatial system (4.3) which is taken as a specific case of Proposition 3.3 from Lui [27] . This result says that there exists a positive, strictly dominant eigenvalue with corresponding strictly positive eigenvector to the finite system, under certain conditions. Additionally, the system has a spreading speed which can be calculated by a formula involving the dominant eigenvalue of $\mathbf{B}_{\mu \mid m}$.

We note that the $m$-dimensional finite system (4.3) has the property that for $0 \leq \rho \leq 1, H(\rho u) \geq \rho H(u)$ since $H(u) / u$ is non-increasing (Hypotheses 4.1). This implies that

$$
\mathbf{Q}_{m}[\rho \mathbf{u}] \geq \rho \mathbf{Q}_{m}[\mathbf{u}]
$$

which is needed for the following lemma to hold.

Let $\mathbf{u}_{* \mid m}$ be the positive steady-state of the finite difference equation system which was described in Chapter 3 Eq. (3.14).

LEMMA 4.1. Suppose Hypotheses 4.1i, ii, $v$ hold and that $H$ is non-decreasing. Suppose $\mathbf{u}_{0 \mid m}(x)$ is continuous, $\mathbf{0}<\mathbf{u}_{0 \mid m}(x)<<\mathbf{u}_{* \mid m}, \mathbf{u}_{0 \mid m}(x)$ is positive on a set with positive measure, and $\mathbf{u}_{0 \mid m}(x)=0$ for sufficiently large $x$.

Then $\mathbf{B}_{\mu \mid m}$ is irreducible and has a positive eigenvalue $\lambda_{* \mid m}(\mu)$ that is no less than the moduli of all other eigenvalues of $\mathbf{B}_{\mu \mid m}$.

Furthermore, $\ln \lambda_{* \mid m}(\mu)$ is convex, $\lambda_{* \mid m}(0)>1$ and

$$
c_{m}^{*}=\inf _{\mu>0} \frac{\ln \lambda_{* \mid m}(\mu)}{\mu}
$$


where ${ }^{*}$ is the spreading speed of system (4.3) in the following sense:

i.

$$
\lim _{n \rightarrow \infty}\left\{\sup _{|x| \geq n\left(c_{m}^{*}+\epsilon\right)}\left|\mathbf{u}_{n \mid m}(x)\right|\right\}=0
$$

ii.

$$
\lim _{n \rightarrow \infty}\left\{\sup _{|x| \leq n\left(c_{m}^{*}-\epsilon\right)}\left|\mathbf{u}_{n \mid m}(x)-\mathbf{u}_{* \mid m}\right|\right\}=0 .
$$

Proof. Follows from Proposition 3.3 in Lui [27].

\subsection{Existence of the Eigenvalue}

The following theorem states that an eigenvalue of $\mathbf{B}_{\mu}$ exists when the origin is unstable (i.e. when Hypothesis 4.1iv) holds).

THEOREM 4.1. Suppose Hypotheses 4.1 hold. Then there exits a strictly positive, simple, eigenvalue $\lambda_{*}(\mu)$ of $\mathbf{B}_{\mu}$ with corresponding strictly positive eigenvector $\boldsymbol{\xi}(\mu)$ given by

$$
\xi^{(j)}(\mu)=\Delta_{j-1} H^{\prime}(0) \frac{K(\mu) \xi^{(1)}(\mu)}{\left(\lambda_{*}(\mu)\right)^{j-1}}
$$

for all $j \in\{2,3, \ldots\}$ and choice of $\xi^{(1)}(\mu)$ where $\Delta_{j}=\prod_{i=0}^{j-1} s_{i}$.

Further, $\lambda_{*}(\mu)>1$ and $\lambda_{*}(\mu)$ is twice continuously differentiable on $\mathbb{R}$.

Proof. We consider the characteristic equation $\mathbf{B}_{\mu} \mathbf{u}=\lambda(\mu) \mathbf{u}$ associated with $\mathbf{B}_{\mu}$. Writing out the equations and solving for each seed bank cohort in terms of $A$ gives 


$$
\begin{aligned}
A & =\frac{\rho_{0} A+a_{0} H^{\prime}(0) K(\mu) A+a_{1} S^{0}+\ldots+a_{m} S^{m-1}+\ldots}{\lambda(\mu)} \\
S^{0} & =\frac{s_{0} H^{\prime}(0) K(\mu)}{\lambda(\mu)} A \\
S^{1} & =\frac{s_{1}}{\lambda(\mu)} S^{0}=\frac{s_{0} s_{1} H^{\prime}(0) K(\mu)}{\lambda(\mu)^{2}} A \\
\vdots & \\
S^{m} & =\frac{s_{m}}{\lambda(\mu)} S^{m-1}=\frac{s_{0} s_{1} s_{2} \ldots s_{m} H^{\prime}(0) K(\mu)}{\lambda(\mu)^{m+1}} A
\end{aligned}
$$

Substituting into the first Eq. (4.7) and dividing by $A$ produces the following infinite series

$$
\begin{aligned}
1 & =\frac{\rho_{o}+a_{0} H^{\prime}(0) K(\mu)}{\lambda(\mu)}+\frac{a_{1} s_{0} H^{\prime}(0) K(\mu)}{\lambda(\mu)^{2}}+\frac{a_{2} s_{0} s_{1} H^{\prime}(0) K(\mu)}{\lambda(\mu)^{3}}+\ldots \\
& =\frac{\rho_{o}}{\lambda(\mu)}+H^{\prime}(0) K(\mu)\left(\frac{a_{0}}{\lambda(\mu)}+\frac{a_{1} \Delta_{1}}{\lambda(\mu)^{2}}+\frac{a_{2} \Delta_{2}}{\lambda(\mu)^{3}}+\ldots\right)
\end{aligned}
$$

where $\Delta_{j}=\prod_{i=0}^{j-1} s_{i}$.

To prove existence of $\lambda_{*}(\mu)>1$ we show that Eq. (4.8) has a solution greater than 1. This approach is very similar to that of Theorem 3.2.

To this end, let $\varphi(\mu)=\frac{1}{\lambda(\mu)}$ and

$$
G(\varphi(\mu))=-1+\rho_{\circ} \varphi(\mu)+H^{\prime}(0) K(\mu)\left(a_{0} \varphi(\mu)+\sum_{i=1}^{\infty} a_{i} \Delta_{i}(\varphi(\mu))^{i+1}\right)
$$

We show there exists $\varphi^{*}(\mu) \in(0,1)$ such that $G\left(\varphi^{*}(\mu)\right)=0$.

Observe that $G(0)=-1<0$. Also, $G(1)>0$ by Hypothesis 4.1 iv and the fact that $K(\mu)>1$. Recalling that $s_{i}=\rho_{d}^{i}\left(1-\beta^{i}\right) \rho_{\nu 2}$ we see that each $\Delta_{i}$ has the term $\left(\rho_{\nu 2}\right)^{i}$. Thus, $G(1)<\infty$ since it is bounded above by a convergent geometric series.

Therefore, by the Intermediate Value Theorem, there exists a solution $\varphi^{*}(\mu) \in(0,1)$ such that $G\left(\varphi^{*}(\mu)\right)=0$. Hence, there is a strictly positive eigenvalue of $\mathbf{B}_{\mu}$ such that $\lambda_{*}(\mu) \in(1, \infty)$. 
We now show that $\lambda_{*}(\mu)$ is simple. Observe that the derivative with respect to $\varphi$ of $\mathrm{Eq}$. (4.9), the characteristic equation of $\mathbf{B}_{\mu}$, is positive when evaluated at $\varphi^{*}(\mu)$. Thus, the multiplicity of the root $\lambda_{*}(\mu)$ is one (i.e. $\lambda_{*}(\mu)$ is simple).

Finally, note that $G(\mu, \varphi(\mu))$ is a power series with an infinite radius of convergence. Hence, using the Implicit Function Theorem and the fact that $\lambda_{*}(\mu)$ is simple, we have that $\lambda_{*}(\mu)$ is twice continuously differentiable on $\mathbb{R}$.

REMARK 4.2. Throughout the remainder of this dissertation we will let $\lambda_{*}(\mu)$ be the strictly positive, simple, eigenvalue of $\mathbf{B}_{\mu}$ with corresponding strictly positive eigenvector $\boldsymbol{\xi}(\mu)$.

\subsection{Properties of the Eigenvalue}

As mentioned in Section 1.3, for a scalar integro-difference equation, the

convexity of the wave speed equation $\phi(\mu)=\frac{1}{\mu} \ln \left(f^{\prime}(0) \int_{-\infty}^{\infty} e^{\mu y} k(y) d y\right)$ is important because it is related to the calculation of the spreading speed. In the following theorem we prove that the eigenvalue $\lambda_{*}(\mu)$ associated with $\mathbf{B}_{\mu}$ is $\log$ convex on $\mathbb{R}$.

THEOREM 4.2. Suppose Hypotheses 4.1 hold. Then $\lambda_{*}(\mu)$ is $\log$ convex on $\mathbb{R}$.

Proof. This proof is similar to that of Lemma 6.4 in [27]. Let $\mu_{1}, \mu_{2} \in \mathbb{R}$ and $0<t<1$. We show that the following inequality holds

$$
\lambda_{*}\left(t \mu_{1}+(1-t) \mu_{2}\right) \leq \lambda_{*}\left(\mu_{1}\right)^{t} \lambda_{*}\left(\mu_{2}\right)^{1-t} .
$$

Let $\boldsymbol{\xi}$ and $\boldsymbol{\eta}$ be eigenvectors such that $\mathbf{B}_{\mu_{1}} \boldsymbol{\xi}=\lambda_{*}\left(\mu_{1}\right) \boldsymbol{\xi}$ and $\mathbf{B}_{\mu_{2}} \boldsymbol{\eta}=\lambda_{*}\left(\mu_{2}\right) \boldsymbol{\eta}$. 
Thus,

$$
\begin{aligned}
& \sum_{j=1}^{\infty} b_{i j}\left(\mu_{1}\right) \frac{\xi_{j}}{\xi_{i}}=\lambda_{*}\left(\mu_{1}\right) \\
& \sum_{j=1}^{\infty} b_{i j}\left(\mu_{2}\right) \frac{\eta_{j}}{\eta_{i}}=\lambda_{*}\left(\mu_{2}\right)
\end{aligned}
$$

where $b_{i j}(\mu)$ is the entry of the matrix $\mathbf{B}_{\mu}$ in the $i$-th row and $j$-th column and $\xi_{j}$ $\left(\eta_{j}\right)$ is the $j$-th component of the eigenvector $\boldsymbol{\xi}(\boldsymbol{\eta})$.

Using Hölder's Inequality and simplifying, we have that for every $i$,

$$
\begin{aligned}
\lambda_{*}\left(\mu_{1}\right)^{t} \lambda_{*}\left(\mu_{2}\right)^{1-t} & =\left(\sum_{j=1}^{\infty} b_{i j}\left(\mu_{1}\right) \frac{\xi_{j}}{\xi_{i}}\right)^{t}\left(\sum_{j=1}^{\infty} b_{i j}\left(\mu_{2}\right) \frac{\eta_{j}}{\eta_{i}}\right)^{1-t} \\
& \geq \sum_{j=1}^{\infty}\left(b_{i j}\left(\mu_{1}\right) \frac{\xi_{j}}{\xi_{i}}\right)^{t}\left(b_{i j}\left(\mu_{2}\right) \frac{\eta_{j}}{\eta_{i}}\right)^{1-t} \\
& =\sum_{j=1}^{\infty}\left(b_{i j}\left(\mu_{1}\right)\right)^{t}\left(b_{i j}\left(\mu_{2}\right)\right)^{1-t} \frac{\chi_{j}}{\chi_{i}} \\
& \geq \frac{\left[\mathbf{B}_{\mu_{1} t+\mu_{2}(1-t)} \chi\right]_{i}}{\chi_{i}}
\end{aligned}
$$

where $\chi_{i}=\xi_{i}^{t} \eta_{i}^{1-t}$.

LEMMA 4.2. $\frac{\left[\mathrm{B}_{\mu} \chi\right]_{i}}{\chi_{i}} \geq \lambda_{*}(\mu)$ for all $\chi \in \ell_{1}$ and $\mu \in \mathbb{R}^{+}$.

Proof. Suppose there exists $\chi \in \ell_{1}$ such that $\mathbf{B}_{\mu} \chi<\lambda_{*}(\mu) \chi$. Let $\mathbf{q}^{T}$ be a left eigenvector of $\mathbf{B}_{\mu}$. Then,

$$
\mathbf{q}^{T} \mathbf{B}_{\mu} \chi<\lambda_{*}(\mu) \mathbf{q}^{T} \chi
$$

But this is a contradiction since $\mathbf{q}^{T} \mathbf{B}_{\mu}=\lambda_{*}(\mu) \mathbf{q}^{T}$.

Thus, by Lemma 4.2 ,

$$
\lambda_{*}\left(\mu_{1}\right)^{t} \lambda_{*}\left(\mu_{2}\right)^{1-t} \geq \lambda_{*}\left(t \mu_{1}+(1-t) \mu_{2}\right)
$$

and we have that $\lambda_{*}(\mu)$ is log convex on $\mathbb{R}$. 
Finally, note that since $\boldsymbol{\xi}, \boldsymbol{\eta} \in \ell_{1}$ then

$$
\|\chi\|=\sum_{i=1}^{\infty}\left|\left(\xi_{i}\right)^{t}\left(\eta_{i}\right)^{1-t}\right| \leq \sum_{i=1}^{\infty}\left(\xi_{i}\right)^{t} \sum_{i=1}^{\infty}\left(\eta_{i}\right)^{1-t}<\infty .
$$

The study of finite nonnegative matrices is extensive and there is a plethora of theory surrounding this topic. We thus consider $\mathbf{B}_{\mu \mid m}$ (see (4.6)) to gain further insight into the properties of $\lambda_{*}$.

Since $\mathbf{B}_{\mu \mid m}$ is irreducible and primitive, then by the Perron-Frobenius Theorem, it has a positive, simple, strictly dominant eigenvalue with corresponding positive right and left eigenvectors. We denote this eigenvalue as $\lambda_{m}^{*}(\mu)$.

The following theorem relates the positive eigenvalue of the infinite matrix $\mathbf{B}_{\mu}$ to the dominant eigenvalues of the finite matrices $\left\{\mathbf{B}_{\mu \mid m}\right\}_{m=1}^{\infty}$.

THEOREM 4.3. Suppose Hypotheses 4.1 hold. Then $\lambda_{m}^{*}(\mu)$ converges uniformly to $\lambda_{*}(\mu)$ on every nonnegative closed interval.

Proof. Let $\epsilon>0$ and $b \in \mathbb{R}^{+}$. We show $\lambda_{m}^{*}(\mu) \rightrightarrows \lambda^{*}(\mu)$ for $\mu \in[0, b]$.

Recall Eq. (4.9), the characteristic equation of $\mathbf{B}_{\mu}$ :

$$
G(\mu, \varphi)=-1+\rho_{o} \varphi+H^{\prime}(0) K(\mu)\left(a_{0} \varphi+\sum_{i=1}^{\infty} a_{i} \Delta_{i} \varphi^{i+1}\right)
$$

Thus,

$$
G\left(\mu, \varphi^{*}\right)=0
$$

where $\varphi^{*}=\frac{1}{\lambda_{*}}$. Similarly, we may obtain the characteristic equation of $\mathbf{B}_{\mu \mid m}$ as:

$$
G_{m}(\mu, \varphi)=-1+\rho_{o} \varphi+H^{\prime}(0) K(\mu)\left(a_{0} \varphi+\sum_{i=1}^{m-1} a_{i} \Delta_{i} \varphi^{i+1}\right)
$$

so that,

$$
G_{m}\left(\mu, \varphi_{m}^{*}\right)=0
$$

where $\varphi_{m}^{*}=\frac{1}{\lambda_{m}^{*}}$. 
We denote the remainder of these characteristic equations as

$$
\begin{aligned}
R_{m}(\mu, \varphi) & :=G(\mu, \varphi)-G_{m}(\mu, \varphi) \\
& =I^{\prime}(0) K(\mu) \sum_{i=m}^{\infty} a_{i} \Delta_{i} \varphi^{i+1}
\end{aligned}
$$

We begin by proving the following three lemmas.

LEMMA 4.3. For every $\epsilon>0$ there exists $M$ such that for all $m>M$

$$
R_{m}\left(\mu, \varphi^{*}\right)<\epsilon
$$

for all $\mu \in[0, b]$.

Proof. Let $\epsilon>0$ and $B=\sup _{\mu \in[0, b]} K(\mu)$. Since $\varphi^{*}(\mu) \leq 1$ and $a_{i}<1$ we have that

$$
\begin{aligned}
R_{m}\left(\mu, \varphi^{*}\right) & =H^{\prime}(0) K(\mu) \sum_{i=m}^{\infty} a_{i} \Delta_{i} \varphi^{*}(\mu)^{i+1} \\
& \leq B H^{\prime}(0) \sum_{i=m}^{\infty} \Delta_{i} .
\end{aligned}
$$

Recall that $\Delta_{i}=\prod_{i=0}^{m-1} s_{i}=\prod_{i=0}^{m-1} \rho_{d}^{i}\left(1-\beta^{i}\right) \rho_{\nu 2}$. Thus, $\sum_{i=m}^{\infty} \Delta_{i}$ is bounded above by the convergent geometric series $\sum_{i=m}^{\infty}\left(\rho_{\nu 2}\right)^{i}$. Therefore, we may choose $M \in \mathbb{N}$ such that for every $m>M, \sum_{i=m}^{\infty} \Delta_{i}<\frac{\epsilon}{B H^{\prime}(0)}$.

Therefore, $R_{m}\left(\mu, \varphi^{*}\right)<\epsilon$ for all $\mu \in[0, b]$.

LEMMA 4.4. For all $\mu \in[0, b]$ we have that

$$
\frac{\partial G_{m}(\mu, \varphi)}{\partial \varphi}>\rho_{o}
$$

Proof. The proof follows directly by differentiating $G_{m}$ term by term and using the fact that $K(\mu)>1$ for all $\mu$ and $\varphi \in(0,1]$.

LEMMA 4.5. Let $\mu>0$. Then $\lambda_{m}^{*}(\mu)<\lambda_{*}(\mu)$ for all $m \in \mathbb{N}$. 
Proof. Let $m \in \mathbb{N}$. Observe that,

$$
G(0)=-1<0
$$

and

$$
G\left(\varphi_{m}^{*}\right)=I^{\prime}(0) K(\mu) \sum_{i=m}^{\infty} a_{i} \Delta_{i}\left(\varphi_{m}^{*}\right)^{i+1}>0 .
$$

Therefore, by the Intermediate Value Theorem there exists a positive root of $G(\varphi)$ which is smaller than $\varphi_{m}^{*}$. Thus, $\varphi^{*}<\varphi_{m}^{*}$ which implies that

$$
\lambda_{m}^{*}<\lambda_{*}
$$

We proceed to prove the theorem. Note that

$$
G\left(\mu, \varphi^{*}\right)=G_{m}\left(\mu, \varphi^{*}\right)+R_{m}\left(\mu, \varphi^{*}\right)=0 .
$$

Let $\epsilon>0$. By Lemma 4.3 we may choose $M \in \mathbb{N}$ such that for all $m>M$,

$$
R_{m}\left(\mu, \varphi^{*}\right)<\frac{\rho_{o}}{\left(\lambda_{*}\right)^{2}} \epsilon
$$

for all $\mu \in[0, b]$.

Note that $G_{m}(\varphi)$ is continuous on $\left[\varphi^{*}, \varphi_{m}^{*}\right]$ and differentiable on $\left(\varphi^{*}, \varphi_{m}^{*}\right)$. Hence, by the Mean Value Theorem, there exists $\bar{\varphi} \in(0, b)$ such that

$$
\left.\frac{\partial G_{m}(\mu, \varphi)}{\partial \varphi}\right|_{\bar{\varphi}}=\frac{\left|G_{m}\left(\mu, \varphi^{*}\right)-G_{m}\left(\mu, \varphi_{m}^{*}\right)\right|}{\left|\varphi^{*}-\varphi_{m}^{*}\right|} .
$$

Lemma 4.4 together with Eqs. (4.12) and (4.13) show that

$$
\begin{aligned}
\left|\varphi^{*}-\varphi_{m}^{*}\right| & =\frac{\left|G_{m}\left(\mu, \varphi^{*}\right)-G_{m}\left(\mu, \varphi_{m}^{*}\right)\right|}{\left.\frac{\partial G_{m}(\mu, \varphi)}{\partial \varphi}\right|_{\bar{\varphi}}} \\
& =\frac{\left|R_{m}\left(\mu, \varphi^{*}\right)\right|}{\left.\frac{\partial G_{m}(\mu, \varphi)}{\partial \varphi}\right|_{\bar{\varphi}}} \\
& <\frac{\epsilon}{\left(\lambda_{*}\right)^{2}} .
\end{aligned}
$$


Since $\varphi^{*}(\mu)=\frac{1}{\lambda_{-}(\mu)}$ then we have that

$$
\left|\lambda_{m}^{*}(\mu)-\lambda_{*}(\mu)\right|<\lambda_{m}^{*}(\mu) \lambda_{*}(\mu) \frac{\epsilon}{\left(\lambda_{*}(\mu)\right)^{2}}
$$

Therefore, by Lemma 4.5,

$$
\left|\lambda_{m}^{*}(\mu)-\lambda_{*}(\mu)\right|<\epsilon
$$

for all $\mu \in[0, b]$.

COROLLARY 4.1. $\phi_{m}(\mu)$ converges uniformly to $\phi(\mu)$ on every nonnegative closed interval where

$$
\phi_{m}(\mu):=\frac{\ln \left(\lambda_{m}^{*}(\mu)\right)}{\mu}
$$

and

$$
\phi(\mu):=\frac{\ln \left(\lambda_{*}(\mu)\right)}{\mu}
$$

Proof. The result follows directly from Theorem 4.3 and the fact that the natural $\log$ is a continuous function.

\subsection{The Spreading Speed}

We are interested in finding an asymptotic rate of spread of the plant population described by the integro-difference system (4.1).

Define

$$
c^{*}:=\inf _{\mu>0}\left\{\frac{\ln \left(\lambda_{*}(\mu)\right)}{\mu}\right\} .
$$

As shown in Theorem 4.2, $\lambda_{*}(\mu)$ is $\log$ convex on $R$. This ensures that the infimum in Eq. (4.14) exists however it could be $\infty$. The following hypothesis avoids this situation.

HYPOTHESES 4.2. Suppose the infimum in Eq. (4.14) is attained at a finite value, $\bar{\mu}$. 
Thus,

$$
c^{*}=\frac{\ln \left(\lambda_{*}(\bar{\mu})\right)}{\bar{\mu}}
$$

which implies that

$$
\lambda_{*}(\bar{\mu})=e^{c^{*} \bar{\mu}} .
$$

We proceed to show that $c^{*}$ is the spreading speed of system (4.1) by showing it is both an upper and a lower bound for the asymptotic rate of spread. We first consider the monotone case and then the non-monotone case.

\section{Monotone Case}

Before we state the theorem we would like to remark that if any single component $u_{0}^{(i)}(x)$ is initially positive at a point, then after $i$ generations, the first $i$ components will be positive at that point as well. More specifically, if $u_{0}^{(i)}\left(x_{0}\right)>0$ for some $x_{0}>0$, then $u_{i}^{(n)}\left(x_{0}\right)>0$ for all $n \in\{1,2, \ldots, i\}$. Thus, by assuming that just one component is initially positive somewhere, we are guaranteed that any finite number of components will be positive after a sufficient number of generations.

THEOREM 4.4. Suppose Hypotheses 4.1 and 4.2 hold and that $H$ is non-decreasing. Then $c^{*}$ is the asymptotic spreading speed of the system (4.1) in the following sense:

If $\mathbf{u}_{0}(x)$ is continuous, $\mathbf{0} \leq \mathbf{u}_{0}(x)<<\mathbf{u}_{*}, u_{0}^{(i)}(x)$ is positive on a set with positive measure for some $i$, and $\mathbf{u}_{0}(x)=\mathbf{0}$ for sufficiently large $x$, then for any $\epsilon>0$ the solution $\mathbf{u}_{n}$ of system (4.1) has the following properties

$i$.

$$
\lim _{n \rightarrow \infty}\left\{\sup _{|x| \geq n\left(c^{*}+\epsilon\right)}\left|\mathbf{u}_{n}(x)\right|\right\}=\mathbf{0},
$$

$i i$.

$$
\lim _{n \rightarrow \infty}\left\{\sup _{|x| \leq n\left(c^{*}-\epsilon\right)}\left|\mathbf{u}_{n}(x)-\mathbf{u}_{*}\right|\right\}=0
$$


Proof. Let $\mathbf{L}[\mathbf{u}]$ be the linearization of $\mathbf{Q}[\mathbf{u}]$ about $\mathbf{0}$. It is easy to see that $\mathbf{L}\left[e^{-\bar{\mu} x} \boldsymbol{\xi}(\bar{\mu})\right]=\mathbf{B}_{\bar{\mu}} e^{-\bar{\mu} x} \boldsymbol{\xi}(\bar{\mu})$ by using the fact that $k(x)$ is symmetric.

We show that Eq. (4.16) holds, i.e. that the number $c^{*}$ is an upper bound for the spreading speed of the system (4.1). Since $\boldsymbol{\xi}(\bar{\mu})>>0$, we may choose a constant $\rho$ such that $\mathbf{u}_{0}(x) \leq \rho e^{-\bar{\mu} x} \boldsymbol{\xi}(\bar{\mu})$ and $\mathbf{u}_{0}(x)$ satisfies the hypotheses of the theorem. Observe that

$$
\begin{aligned}
\mathbf{u}_{1}(x)=\mathbf{Q}\left[\mathbf{u}_{0}(x)\right] & \leq \mathbf{L}\left[\mathbf{u}_{0}(x)\right] \\
& \leq \rho \mathbf{L}\left[e^{-\bar{\mu} x} \boldsymbol{\xi}(\bar{\mu})\right] \\
& =\rho \mathbf{B}_{\bar{\mu}} e^{-\bar{\mu} x} \boldsymbol{\xi}(\bar{\mu}) \\
& =\rho \lambda_{*}(\bar{\mu}) e^{-\bar{\mu} x} \boldsymbol{\xi}(\bar{\mu}) \\
& =\rho e^{-\bar{\mu}\left(x-c^{*}\right)} \boldsymbol{\xi}(\bar{\mu}) .
\end{aligned}
$$

By induction we have that

$$
\mathbf{u}_{n}(x) \leq \rho e^{-\tilde{\mu}\left(x-n c^{*}\right)} \boldsymbol{\xi}(\bar{\mu})
$$

Thus, for all $x \geq n\left(c^{*}+\epsilon\right)$,

$$
\begin{aligned}
\mathbf{u}_{n}(x) & \leq \rho e^{-\bar{\mu}\left(n\left(c^{*}+\varepsilon\right)-n c^{*}\right)} \boldsymbol{\xi}(\bar{\mu}) \\
& =\rho e^{-\bar{\mu} n \epsilon} \boldsymbol{\xi}(\bar{\mu}) .
\end{aligned}
$$

Therefore,

$$
\sup _{x \geq n\left(c^{*}+\epsilon\right)}\left|\mathbf{u}_{n}(x)\right| \leq \rho e^{-\bar{\mu} n \epsilon} \boldsymbol{\xi}(\bar{\mu})
$$

and so,

$$
\lim _{n \rightarrow \infty}\left\{\sup _{x \geq n\left(c^{*}+\epsilon\right)}\left|\mathbf{u}_{n}(x)\right|\right\}=0 .
$$

We may use the same procedure for the case when $x \leq-n\left(c^{*}+\epsilon\right)$ to obtain Eq. (4.16).

We now show that Eq. (4.17) holds, i.e. that $c^{*}$ is a lower bound for the spreading speed. First note that Eq. (4.17) is equivalent to the following statement: 
For every c $>0$ there exists $N \in \mathbb{N}$ such that for all $n>N$

$$
\left|\mathbf{u}_{n}(x)-\mathbf{u}_{*}\right|<\epsilon
$$

for all $|x| \leq n\left(c^{*}-\delta\right)$.

We will use this statement to prove the theorem.

Choose $\mathbf{u}_{0}(x)$ such that it satisfies the assumptions in the theorem. For given $m$, let

$$
\mathbf{u}_{0 \mid m}(x)= \begin{cases}u_{0}^{(i)}(x) & \text { if } i \in\{1, \ldots, m\} \\ 0 & \text { if } i \in\{m+1, m+2, \ldots\}\end{cases}
$$

Thus, for any $m$,

$$
\mathbf{u}_{n}(x) \geq \mathbf{u}_{n \mid m}(x) \text { for all } n
$$

where $\mathbf{u}_{n \mid m}$ is the solution of the m-dimensional recursion (4.3).

Note that since $u_{0}^{(i)}(x)$ is positive on a set with positive measure for some $i$, then there exists $N$ such that $u_{N}^{(j)}(x)$ is positive on a set with positive measure for each $j \in\{0,1, \ldots, m\}$. Thus, without loss of generality, we may assume that the initial distribution $\mathbf{u}_{0 \mid m}(x)$ is positive on a set with positive measure.

Let $c_{m}^{*}:=\inf _{\mu>0} \frac{\ln \lambda_{m}^{*}(\mu)}{\mu}$. By Lemma $4.1, c_{m}^{*}$ is the spreading speed of the $m$-dimensional recursion (4.3) with initial distribution $\mathbf{u}_{0 \mid m}(x)$.

Now, let $\epsilon>0$ and $\delta>0$. Since $c^{*}$ is attained at a finite value $\bar{\mu}$ then, by Corollary 4.1 , we have that $c_{m}^{*} \rightarrow c^{*}$. Thus, we may choose $M_{1} \in \mathbb{N}$ such that for all $m>M_{1}$,

$$
\left|c_{m}^{*}-c^{*}\right|<\delta
$$

By Theorem 3.6 we may choose $M_{2} \in \mathbb{N}$ such that for all $m>M_{2}$,

$$
\left|\mathbf{u}_{* \mid m}-\mathbf{u}_{*}\right|<\frac{c}{2}
$$

Let $M=\max \left\{M_{1}, M_{2}\right\}$. By Lemma 4.1 we may choose $N \in \mathbb{N}$ such that for all $n>N$,

$$
\left|\mathbf{u}_{n \mid M}(x)-\mathbf{u}_{* \mid M}\right|<\frac{\epsilon}{2} \text { for all }|x| \leq n\left(c_{M}^{*}-2 \delta\right)
$$


Thus Eq. (4.19) and (4.21) imply that

$$
\left|\mathbf{u}_{n \mid M}(x)-\mathbf{u}_{* \mid M}\right|<\frac{\epsilon}{2} \text { for all }|x| \leq n\left(c^{*}-\delta\right)
$$

Equations (4.18), (4.20), and (4.22) imply that for all $|x| \leq n\left(c^{*}-\delta\right)$,

$$
\begin{aligned}
\mathbf{0}<\mathbf{u}_{*}-\mathbf{u}_{n}(x) & =\left(\mathbf{u}_{* \mid M}-\mathbf{u}_{n \mid M}(x)\right)+\left(\mathbf{u}_{n \mid M}(x)-\mathbf{u}_{n}(x)\right)+\left(\mathbf{u}_{*}-\mathbf{u}_{* \mid M}\right) \\
& <\epsilon
\end{aligned}
$$

Therefore Eq. (4.17) holds and the theorem is proved.

\section{Non-monotone Case}

We now show that $c^{*}$ is the spreading speed for the system (4.1) when $H$ may not be monotone. We first introduce some auxiliary functions:

Define the function

$$
H^{+}(u):=\max _{0 \leq v \leq u} H(v)
$$

for $0 \leq u \leq \kappa_{0}$ (denoted by $G(u, 0)$ in [43]). $H^{+}(u)$ is a continuous and nondecreasing function, and $0 \leq H(u) \leq H^{+}(u) \leq \kappa_{0}$ for $0 \leq u \leq \kappa_{0}$. Note that Hypothesis 4.1ii shows that $H^{+}(u)=H(u)$ for $u \leq d$.

We showed in Theorem 3.4 that $\mathbf{u}_{*}=\left(A_{*} S_{*}^{0} S_{*}^{1} \ldots\right)$ is the positive steadystate of the non-spatial system (3.1). We find, by a similar argument, that there exists a strictly positive steady-state to the system (3.1) if we replace the growth function $H(u)$ by $H^{+}(u)$. Call this positive solution $\sigma_{+}$and the first component of this positive solution $\kappa$.

Now define the function

$$
H^{-}(u):=\min _{u \leq v \leq \kappa} H(v) \quad \text { for } 0 \leq u \leq \kappa
$$

(This function is denoted by $G(u, \alpha)$ in [43].) Clearly, $H^{-}(u) \leq H(u)$ for $0 \leq u \leq$ $\kappa$. Also, $H^{-}(u)$ is non-decreasing and continuous on $[0, \kappa]$. Again, we can show 
there exists a strictly positive steady-state to the non-spatial system (3.1) if we replace the growth function $I(u)$ by $I^{-}(u)$. Denote this positive solution as $\sigma_{-}$ and the first component of this positive solution $\sigma_{-}$.

Let $\mathbf{Q}^{+}$and $\mathbf{Q}^{-}$be defined by (4.2) with $H$ replaced by $H^{+}$and $H^{-}$, respectively. Since $H^{-}(u) \leq H(u) \leq H^{+}(u)$ then

$$
\mathrm{Q}^{-}[\mathbf{u}] \leq \mathrm{Q}[\mathbf{u}] \leq \mathrm{Q}^{+}[\mathbf{u}]
$$

for $\mathbf{u}(x)$ continuous and $\mathbf{0} \leq \mathbf{u}(x) \leq \sigma_{+}$.

THEOREM 4.5. Suppose Hypotheses 4.1 and 4.2 hold. Then $c^{*}$ is the asymptotic spreading speed of the system (4.1) in the following sense:

If $\mathbf{0} \leq \mathbf{u}_{0}(x)<<\mathbf{u}_{*}, \mathbf{u}_{0}(x)$ is continuous for all $x, u_{0}^{(i)}(x)$ is positive on a set with positive measure for some $i$, and $\mathbf{u}_{0}(x)=\mathbf{0}$ for sufficiently large $x$, then for any $\epsilon>0$ the solution $\mathbf{u}_{n}$ of system (4.1) has the following properties

$i$.

$$
\mathbf{u}_{n}(x) \leq \boldsymbol{\sigma}_{+} \text {for all } x \text { and } n
$$

ii.

$$
\lim _{n \rightarrow \infty}\left\{\sup _{|x| \geq n\left(c^{*}+\varepsilon\right)}\left|\mathbf{u}_{n}(x)\right|\right\}=0,
$$

iii.

$$
\liminf _{n \rightarrow \infty}\left\{\inf _{|x| \leq n\left(c^{*}-\epsilon\right)}\left|\mathbf{u}_{n}(x)\right|\right\} \geq \boldsymbol{\sigma}_{-}
$$

Proof. Case i: Let $\mathbf{v}_{n}^{+}(x)$ and $\mathbf{v}_{n}^{-}(x)$ be solutions of the recursions $\mathbf{v}_{n+1}^{+}(x)=$ $\mathbf{Q}^{+}\left[\mathbf{v}_{n}^{+}\right](x)$ and $\mathbf{v}_{n+1}^{-}(x)=\mathbf{Q}^{-}\left[\mathbf{v}_{n}^{-}\right](x)$, respectively. Then the Comparison Principle says that

$$
\mathbf{v}_{n}^{-}(x) \leq \mathbf{u}_{n}(x) \leq \mathbf{v}_{n}^{+}(x)
$$

Since $\mathbf{v}_{n}^{+}(x) \leq \boldsymbol{\sigma}_{+}$, then $\mathbf{u}_{n}(x) \leq \boldsymbol{\sigma}_{+}$for all $x$ and $n$. 
Case ii and iii: Note that $I I, I^{+}$and $\|^{-}$have the same linearization at 0 . Thus, they each have the same linearized moment generating matrix $\mathbf{B}_{\mu}$. Hence, by Theorem $4.4, \mathbf{v}_{n}^{+}$and $\mathbf{v}_{n}^{-}$have the same sprearling speed $c^{*}$ since $H^{+}$ and $\mathrm{H}^{-}$are non-decreasing. In particular, Eq. (4.23) is satisfied with u replaced by $\mathbf{v}^{+}$and Eq. (4.24) is satisfied with $\mathbf{u}$ replaced by $\mathbf{v}^{-}$.

\subsection{Traveling Wave Solutions}

In this section we prove the existence of traveling wave solutions when $c \geq c^{*}$ for the recursion (4.1). Proofs of these theorems make use of some limiting properties of the recursion when the growth function is bounded above by a constant. We state these limiting properties here, as a lemma:

LEMMA 4.6. Let $\mathbf{u}_{n}(x)$ be described by the recursion (4.1) for given continuous $\mathbf{u}_{0}(x)$. Suppose there exists function $\mathbf{u}(x)$ which is the limit of $\mathbf{u}_{n}(x)$ as $n \rightarrow \infty$ and that $\mathbf{u}(x), \mathbf{u}_{0}(x)$ are bounded above by $\mathrm{U} \in \ell_{1}$. Further, suppose that the growth function $H(u(x))$ is bounded above by $\mathbf{U}$ for all $n \in \mathbb{N}$ and $x \in \mathbb{R}$. Then

i. $\lim _{n \rightarrow \infty} \int_{\mathbb{R}} k(x-y) H\left(u_{n}^{(1)}(y)\right) d y=H\left(u^{(1)}(x)\right)$

ii. $\lim _{x \rightarrow-\infty} \int_{\mathbb{R}} k(x-y) H\left(u^{(1)}(y)\right) d y=H\left(u^{(1)}(-\infty)\right)$,

iii. $\lim _{n \rightarrow \infty} \sum_{i=1}^{\infty} a_{i} u_{n}^{(i+1)}(x)=\sum_{i=1}^{\infty} a_{i} u^{(i+1)}(x)$,

iv. $\lim _{x \rightarrow-\infty} \sum_{i=1}^{\infty} a_{i} u^{(i+1)}(x)=\sum_{i=1}^{\infty} a_{i} u^{(i+1)}(-\infty)$

Proof. Statements (i.) and (ii.) are true by an application of the dominated convergence theorem with dominating function $k(x-y) U^{(1)}$.

To prove statement (iii.) let $\delta>0$, fix $x \in \mathbb{R}$ and note that $\sum_{i=1}^{\infty}\left|u^{(i)}(x)\right|<$ $\infty, \sum_{i=1}^{\infty}\left|u_{n}^{(i)}(x)\right|<\infty$ for all $n \in \mathbb{N}$ since both $\mathbf{u}(x)$ and $\mathbf{u}_{n}(x)$ are bounded above by $\mathrm{U}$ which has a finite norm. 
Thus we may choose $M_{1}, M_{2} \in \mathbb{N}$ such that

$$
\sum_{i=M_{1}}^{\infty}\left|u_{n}^{(i)}(x)\right|<\frac{\delta}{3}
$$

and

$$
\sum_{i=M_{2}}^{\infty}\left|u^{(i)}(x)\right|<\frac{\delta}{3} .
$$

Let $M=\max \left\{M_{1}, M_{2}\right\}$. It is easy to show (and well known) that for the finite sum, we may interchange the limit and summation so that $\lim _{n \rightarrow \infty} \sum_{i=1}^{M} u_{n}^{(i)}(x)=$ $\sum_{i=1}^{\infty} u^{(i)}(x)$. Thus, choose $N \in \mathbb{N}$ such that for all $n>N$,

$$
\sum_{i=1}^{M}\left|a_{i} u_{n}^{(i)}(x)-a_{i} u^{(i)}(x)\right|<\frac{\delta}{3} .
$$

Noting that $a_{i}<1$ for all $i$ we have that for all $n>N$,

$$
\begin{aligned}
\left|\sum_{i=1}^{\infty} a_{i} u_{n}^{(i)}-\sum_{i=1}^{\infty} a_{i} u^{(i)}\right| & \leq \sum_{i=1}^{M}\left|a_{i} u_{n}^{(i)}-a_{i} u^{(i)}\right|+\sum_{i=M+1}^{\infty}\left|a_{i} u_{n}^{(i)}\right|+\sum_{i=M+1}^{\infty}\left|a_{i} u^{(i)}\right| \\
& <\delta
\end{aligned}
$$

which proves statement (iii.). Statement (iv.) may be shown in a similar manner to statement (iii.) by noting that $\sum_{i=1}^{\infty}\left|u^{(i)}(-\infty)\right|<\infty$.

\section{Monotone Case}

We first consider the case when $\mathbf{Q}$ is order preserving which is equivalent to assuming that the growth function $H(A)$ is non-decreasing.

THEOREM 4.6. Let Hypotheses 4.1 and 4.2 be satisfied and assume $H(A)$ is nondecreasing. Then the following hold for the spatial system (4.1):

1. For $c \geq c^{*}$ there exists a non-increasing traveling wave $\mathbf{u}_{n}(x)=\mathbf{w}(x-n c)$ with $\mathbf{w}(+\infty)=\mathbf{0}$ and $\mathbf{w}(-\infty)=\mathbf{u}_{*}$.

2. A non-increasing traveling wave solution $\mathbf{w}(x-n c)$ with $\mathbf{w}(\infty)=0$ and $\mathbf{w}(-\infty)=\mathbf{u}_{*}$ does not exist if $c<c^{*}$. 
Proof. First note that since $I(A)$ is non-decreasing, then $\mathrm{Q}$ is order preserving. Case 2: Let $c<c^{*}$. For contradiction, suppose that $\mathbf{w}(x)$ is a traveling wave solution of system (4.1) with speed $c$ such that $\mathbf{w}(-\infty)=\mathbf{u}_{*}$ and $\mathbf{w}(\infty)=\mathbf{0}$.

We choose an initial distribution below the traveling wave, $\mathbf{u}_{0}(x)<\mathbf{w}(x)$, such that $\mathbf{0} \leq \mathbf{u}_{0}(x)<<\mathbf{u}_{*}, \mathbf{u}_{0}(x)$ is continuous for all $x$, and $\mathbf{u}_{0}(x)=\mathbf{0}$ for sufficiently large $x$. Using the order preserving property of operator $\mathbf{Q}$, we have that for all $n \in \mathbb{N}$,

$$
\mathbf{u}_{n}(x) \leq \mathbf{w}(x-n c)
$$

By Theorem 4.4, system (4.1) with initial distribution $\mathbf{u}_{0}(x)$ has a spreading speed $c^{*}$ as defined in the theorem. Thus, for every $\epsilon>0$,

$$
\lim _{n \rightarrow \infty} \sup _{|x| \leq n\left(c^{*}-\varepsilon\right)}\left\{\left|\mathbf{u}_{n}(x)-\mathbf{u}_{*}\right|\right\}=0
$$

We find a contradiction by letting $\epsilon=\frac{c^{*}-c}{2}, N \in \mathbb{N}$ and $x_{n}=n\left(c^{*}-\epsilon\right)$. Thus, by the definition of $c^{*}$,

$$
\lim _{n \rightarrow \infty} \mathbf{u}_{n}\left(x_{n}\right)=\mathbf{u}_{*}
$$

Also,

$$
\begin{aligned}
\lim _{n \rightarrow \infty} \mathbf{w}\left(x_{n}-n c\right) & =\lim _{n \rightarrow \infty} \mathbf{w}\left(\left(n\left(c^{*}-c-\epsilon\right)\right)\right. \\
& =\lim _{n \rightarrow \infty} \mathbf{w}\left(n\left(\frac{c^{*}-c}{2}\right)\right) \\
& =\mathbf{w}(+\infty) \\
& =0
\end{aligned}
$$

Eqs. (4.26) and (4.27) imply that there is an $N \in \mathbb{N}$ such that

$$
\mathbf{u}_{N}\left(x_{N}\right)>\mathbf{w}\left(x_{N}-N c\right)
$$

which contradicts Eq. (4.25). 
Case 1: For $c>c^{*}$, let $\mu_{c}$ denote the smallest solution to $c=\phi(\mu)$. Observe that the linearization of $\mathbf{Q}$ about $\mathbf{0}$ is given by

$$
\mathbf{L}[\mathbf{u}](x)=\left(\begin{array}{c}
\rho_{o} u^{(1)}(x)+a_{0} H^{\prime}(0) \int_{-\infty}^{\infty} k(x-y) u^{(1)}(y) d y+\sum_{i=1}^{\infty} a_{i} u^{(i+1)}(x) \\
s_{0} H^{\prime}(0) \int_{-\infty}^{\infty} k(x-y) u^{(1)}(y) d y \\
s_{1} u^{(2)}(x) \\
\vdots
\end{array}\right)
$$

Define

$$
\mathbf{w}^{+}(x)=e^{-\mu_{c} x} \boldsymbol{\xi}\left(\mu_{c}\right)
$$

Since $\mathbf{B}_{\mu} e^{-\mu x} \boldsymbol{\xi}(\mu)=\mathbf{L}\left[e^{-\mu x} \boldsymbol{\xi}(\mu)\right]$ and $\lambda(\mu)=e^{\mu c}$ then

$$
\begin{aligned}
\mathbf{L}\left[\mathbf{w}^{+}\right](x) & =\mathbf{L}\left[e^{-\mu_{c} x} \boldsymbol{\xi}\left(\mu_{c}\right)\right] \\
& =\mathbf{B}_{\mu_{c}} e^{-\mu_{c} x} \boldsymbol{\xi}\left(\mu_{c}\right) \\
& =\lambda\left(\mu_{c}\right) e^{-\mu_{c} x} \boldsymbol{\xi}\left(\mu_{c}\right) \\
& =e^{-\mu_{c}(x-c)} \boldsymbol{\xi}\left(\mu_{c}\right) \\
& =\mathbf{w}^{+}(x-c) .
\end{aligned}
$$

Now, $\mathbf{Q}\left[\min \left\{\mathbf{w}^{+}, \mathbf{u}_{*}\right\}\right] \leq \mathbf{w}^{+}(x-c)$ since $\mathbf{Q}\left[\min \left\{\mathbf{w}^{+}, \mathbf{u}_{*}\right\}\right] \leq \mathbf{L}\left[\mathbf{w}^{+}\right]=\mathbf{w}^{+}(x-c)$. Also, $\mathrm{Q}\left[\min \left\{\mathbf{w}^{+}, \mathbf{u}_{*}\right\}\right] \leq \mathrm{Q}\left[\mathbf{u}_{*}\right]=\mathbf{u}_{*}$. Therefore,

$$
\mathbf{Q}\left[\min \left\{\mathbf{w}^{+}, \mathbf{u}_{*}\right\}\right](x) \leq \min \left\{\mathbf{w}^{+}(x-c), \mathbf{u}_{*}\right\}
$$

Define

$$
\mathbf{v}(x)=\max \left\{\epsilon\left[e^{-\mu_{c} x} \boldsymbol{\xi}\left(\mu_{c}\right)-e^{-s x} \boldsymbol{\xi}(s)\right], \mathbf{0}\right\}
$$

where $\epsilon$ is a small positive number and $\mu_{c}<s<\min \left\{\bar{\mu}, 2 \mu_{c}\right\}$. Choose $\xi^{(1)}\left(\mu_{c}\right)>$ $\xi^{(1)}(s)$ so that $\mathbf{v}(0)>0$.

In Theorem 4.1 we showed that $\lambda_{*}(\mu)$ is twice continuously differentiable and thus by Lemma 6.5 in [27] we have that $\phi(\mu)$ strictly decreases for $0<\mu<\bar{\mu}$. Hence, $c>\phi(s)$. 
By Hypotheses 4.1 we have that

$$
\mathbf{Q}[\mathbf{v}](x) \geq \mathbf{L}[\mathbf{v}]-d\left(\begin{array}{c}
a_{0} \int_{-\infty}^{\infty} k(x-y)\left(v^{(1)}(y)\right)^{2} d y \\
s_{0} \int_{-\infty}^{\infty} k(x-y)\left(v^{(1)}(y)\right)^{2} d y \\
0 \\
\vdots
\end{array}\right)
$$

Using $\epsilon\left[e^{-\mu_{c} x} \boldsymbol{\xi}\left(\mu_{c}\right)-e^{-s x} \boldsymbol{\xi}(s)\right] \leq \mathbf{v}(x) \leq \epsilon e^{-\mu_{c} x} \boldsymbol{\xi}\left(\mu_{c}\right)$ and $\int_{-\infty}^{\infty} k(x-y) e^{-\mu y} \xi^{(1)}(\mu) d y=$ $e^{-\mu x} \xi^{(1)}(\mu) K(\mu)$, we have that

$$
\begin{aligned}
& \mathbf{L}[\mathbf{v}](x) \geq \mathbf{L}\left[\epsilon\left(e^{-\mu_{c} x} \boldsymbol{\xi}\left(\mu_{c}\right)-e^{-s x} \boldsymbol{\xi}(s)\right)\right] \\
& =\epsilon \cdot\left[\mathbf{B}_{\mu_{c}} e^{-\mu_{c} x} \boldsymbol{\xi}\left(\mu_{\mathrm{c}}\right)-\mathbf{B}_{s} e^{-s x} \boldsymbol{\xi}(s)\right] \\
& =\epsilon\left[\lambda\left(\mu_{c}\right) e^{-\mu_{c} x} \boldsymbol{\xi}\left(\mu_{c}\right)-\lambda(s) e^{-s x} \boldsymbol{\xi}(s)\right] \\
& =\epsilon\left[e^{-\mu_{c}(x-c)} \boldsymbol{\xi}\left(\mu_{c}\right)-e^{-s(\boldsymbol{x}-\phi(s))} \boldsymbol{\xi}(s)\right] \\
& =\epsilon\left[e^{-\mu_{c}(x-c)} \boldsymbol{\xi}\left(\mu_{c}\right)-e^{-s(x-c)} \boldsymbol{\xi}(s)+e^{-s(x-c)} \boldsymbol{\xi}(s)\left(1-e^{-s(c-\phi(s))}\right)\right] \text {, }
\end{aligned}
$$

and

$$
\begin{aligned}
\int_{-\infty}^{\infty} k(x-y)\left(v^{(1)}(y)\right)^{2} d y & \leq \int_{-\infty}^{\infty} k(x-y)\left(\epsilon e^{-\mu_{c} y} \xi^{(1)}\left(\mu_{c}\right)\right)^{2} d y \\
& =\epsilon^{2}\left(\xi^{(1)}\left(\mu_{c}\right)\right)^{2} K\left(2 \mu_{c}\right) e^{-2 \mu_{c} x}
\end{aligned}
$$

It follows from this and Eq. (4.29) that

$$
\begin{aligned}
\mathbf{Q}[\mathbf{v}](x) \geq \epsilon & {\left[e^{-\mu_{c}(x-c)} \boldsymbol{\xi}\left(\mu_{c}\right)-e^{-s(x-c)} \boldsymbol{\xi}(s)\right]+\epsilon e^{-s(x-c)}\left[1-e^{-s(c-\phi(s))}-\right.} \\
& \left.-\operatorname{diag}\left(a_{0} B, s_{0} B, 0, \ldots\right)\right] \boldsymbol{\xi}(s)
\end{aligned}
$$

where

$$
B=\epsilon d \max _{i=1,2}\left\{\frac{\xi^{(i)}\left(\mu_{c}\right)}{\xi^{(i)}(s)}\right\} \xi^{(1)}\left(\mu_{c}\right) K\left(2 \mu_{c}\right) e^{-\left[2 \mu_{c}-s\right] x-s c}
$$

Since $x<2 \mu_{c}$ and $c>\phi(s)$, then for sufficiently small $\epsilon$,

$$
\mathbf{Q}[\mathbf{v}](x) \geq \epsilon\left[e^{-\mu_{c}(x-c)} \boldsymbol{\xi}\left(\mu_{c}\right)-e^{-s(x-c)} \boldsymbol{\xi}(s)\right]
$$


for all $x \geq 0$.

Observe that there exists a number $x_{0}<0$ such that $\mathbf{v}(x) \equiv 0$ for $x \leq x_{0}$. Thus, for sufficiently small $\epsilon>0$, Eq. (4.30) holds for $x_{0} \leq x \leq 0$. Since $\mathrm{Q}[\mathbf{v}](x) \geq \mathbf{0}$ for all $x$ and $\mathbf{v}(x) \equiv \mathbf{0}$ for $x<x_{0}$, then for all $x \in \mathbb{R}$,

$$
\mathbf{Q}[\mathbf{v}](x) \geq \max \left\{c\left[e^{-\mu_{c}(x-c)} \boldsymbol{\xi}\left(\mu_{c}\right)-e^{-s(x-c)} \boldsymbol{\xi}(s)\right], \mathbf{0}\right\}=\mathbf{v}(x-c)
$$

Observe that the $i$-th component of $\left(e^{-\mu_{c}(x-c)} \boldsymbol{\xi}\left(\mu_{c}\right)-e^{-s(x-c)} \boldsymbol{\xi}(s)\right)$ has a finite positive global maximum at a finite number determined by $\mu_{c}, s, \xi^{(i)}\left(\mu_{c}\right)$ and $\xi^{(i)}(s)$. Thus, for sufficiently small $\epsilon$,

$$
\min \left(\mathbf{w}^{+}(x), \mathbf{u}_{*}\right) \geq \mathbf{v}(x)
$$

Define operator $\mathbf{Q}_{c}[\mathbf{u}](x):=\mathbf{Q}[\mathbf{u}](x+c)$. Let $\mathbf{z}_{0}(x)=\min \left(\mathbf{w}^{+}(x), \mathbf{u}_{*}\right)$ and $\mathbf{z}_{n+1}(x)=\mathbf{Q}_{c}\left[\mathbf{z}_{n}\right](x)$ for $n=0,1,2, \ldots$

From Eqs. (4.28), (4.31), (4.32) and the fact that $\mathrm{Q}$ is order preserving we have that

$$
\mathbf{z}_{0}(x) \geq \mathbf{Q}_{c}\left[\mathbf{z}_{0}\right](x) \geq \mathbf{Q}_{c}[\mathbf{v}](x) \geq \mathbf{v}(x)
$$

Induction shows that

$$
\mathrm{z}_{0}(x) \geq \mathrm{z}_{1}(x) \geq \mathrm{z}_{2}(x) \geq \ldots \geq \mathrm{v}(x)
$$

Therefore, $\mathbf{z}_{n}(x)$ converges to some function $\mathbf{w}(x)$ such that

$$
\min \left(\mathbf{w}^{+}(x), \mathbf{u}_{*}\right) \geq \mathbf{w}(x) \geq \mathbf{v}(x)
$$

We now show that $\mathbf{w}(x)=\mathbf{Q}_{c}[\mathbf{w}](x)$ meaning that $\mathbf{w}(x-n c)$ is a traveling wave solution. To this end, we take limits on both sides of the recursion $z_{n+1}^{(i)}(x)=$ 
$Q_{c}^{(i)}\left[z_{n}^{(i)}\right](x)$ for $i=3,4, \ldots$ to obtain

$$
\begin{aligned}
w^{(i)}(x)=\lim _{n \rightarrow \infty} z_{n+1}^{(i)}(x) & =\lim _{n \rightarrow \infty} Q_{c}^{(i)}\left[z_{n}^{(i)}\right](x) \\
& =\lim _{n \rightarrow \infty}\left[s_{i-2} z_{n}^{(i-1)}(x+c)\right] \\
& =s_{i-2} \lim _{n \rightarrow \infty} z_{n}^{(i-1)}(x+c) \\
& =s_{i-2} w_{n}^{(i-1)}(x+c) \\
& =Q_{c}^{(i)}\left[w^{(i)}\right](x) .
\end{aligned}
$$

The linearity of these functions allow us to move the limit inside the operator. By Lemma 4.6.i and 4.6.iii ( with $\mathbf{U}=\mathbf{u}_{*}$ ) we may also move the limit inside the operator of the nonlinear functions (when $i=1,2$ ) which shows that $\mathbf{w}(x-n c$ ) is a traveling wave solution.

Since $\mathbf{z}_{0}(x) \geq \mathbf{w}(x) \geq \mathbf{v}(x) \geq \mathbf{0}$ and $\mathbf{z}_{0}(x) \rightarrow 0$ as $x \rightarrow \infty$, we have that

$$
\mathbf{w}(\infty)=0
$$

Since $\mathbf{w}(x)$ is non-increasing and bounded above by $\mathbf{u}_{*}$, then the limit $\lim _{x \rightarrow-\infty} \mathbf{w}(x)$ exists, call it $\mathbf{w}(-\infty)$.

We now show that $\lim _{x \rightarrow-\infty} \mathbf{Q}_{c}[\mathbf{w}](x)=\mathbf{Q}[\mathbf{w}](-\infty)$. It is easy to see that this is true for the linear components of $\mathbf{Q}_{c}$ by observing that for $i=3,4, \ldots$,

$$
\begin{aligned}
\lim _{x \rightarrow-\infty} Q_{c}^{(i)}\left[w^{(i)}\right](x) & =\lim _{x \rightarrow-\infty}\left[s_{i-2} w^{(i-1)}(x+c)\right] \\
& =s_{i-2} \lim _{x \rightarrow-\infty} w^{(i-1)}(x+c) \\
& =s_{i-2} w^{(i-1)}(-\infty) \\
& =Q^{(i)}\left[w^{(i)}\right](-\infty) .
\end{aligned}
$$

This is true for the nonlinear components by Lemma 4.6.ii and 4.6.iv (with $\mathrm{U}=$ $\left.\mathbf{u}_{*}\right)$.

Hence, $\mathbf{w}(-\infty)=\mathbf{Q}[\mathbf{w}](-\infty)$ and so $\mathbf{w}(-\infty)$ is a steady-state of the difference equation system (2.1). But $\mathbf{w}(-\infty)$ cannot be the extinction steady-state 
since $0<w^{(1)}(0) \leq w^{(1)}(0)$ and $w^{(1)}(x)$ is non-increasing. Therefore it must be the positive steady-state of (2.1) so that

$$
\mathbf{w}(-\infty)=\mathbf{u}_{*}
$$

Finally, we show there exists a traveling wave solution with speed $c^{*}$. Any traveling wave solution of the recursion (4.1) with speed $c$ must satisfy $\mathbf{w}(x)=$ $\mathbf{Q}_{c}[\mathbf{w}](x)$ or equivalently:

$$
\begin{aligned}
A(x-c) & =\rho_{0} A(x)+a_{0} \int_{\mathbb{R}} k(x-y) H(A(y)) d y+\sum_{i=1}^{\infty} a_{i} S^{i-1}(x) \\
S^{0}(x-c) & =s_{0} \int_{\mathbb{R}} k(x-y) H(A(y)) d y \\
S^{1}(x-c) & =s_{1} S^{0}(x) \\
\vdots & \\
S^{m}(x-c) & =s_{m} S^{m-1}(x)
\end{aligned}
$$

where $\mathbf{w}=\left(A, S^{0}, S^{1}, \ldots\right)$. This implies that

$$
\begin{aligned}
S^{m}(x) & =s_{m} S^{m-1}(x+c) \\
& =s_{m} s_{m-1} \ldots s_{1} S^{0}(x+m c) \\
& =s_{m} s_{m-1} \ldots s_{1} s_{0} \int_{\mathbb{R}} k((x+(m+1) c)-y) H(A(y)) d y \\
& =\Delta_{m} \int_{\mathbb{R}} k((x+(m+1) c)-y) H(A(y)) d y .
\end{aligned}
$$

where $\Delta_{m}=\prod_{i=0}^{m} s_{i}$. Hence system (4.33) may be reduced to:

$$
\begin{aligned}
A(x-c)= & \rho_{o} A(x)+a_{0} \int_{\mathbb{R}} k(x-y) H(A(y)) d y+ \\
& +\sum_{i=1}^{\infty} a_{i} \Delta_{i-1} \int_{\mathbb{R}} k(x+i c-y) H(A(y)) d y
\end{aligned}
$$

This shows that the traveling wave system (4.33) is equivalent to the scalar equation (4.35) with $S_{m}(x)$ in (4.33) given by (4.34). 
The problem now becomes establishing the existence of traveling waves for the scalar problem (4.35) when $r=c^{*}$.

'To this end, choose a sequence of positive numbers $\left\{c_{n}\right\}_{n=1}^{\infty}$ which converge to $c^{*}$ from above, so that $c_{n}>c^{*}$ for all $n \in \mathbb{N}$ and $\lim _{n \rightarrow \infty} c_{n}=c^{*}$. We have just shown that for each $n$ there exists a traveling wave solution $\mathbf{w}_{c_{n}}(x)$ to system (4.1) with speed $c_{n}$. Thus the scalar traveling wave equation (4.35) implies that for each $n$

$$
\begin{aligned}
A_{c_{n}}\left(x-c_{n}\right)= & \rho_{o} A_{c_{n}}(x)+a_{0} \int_{\mathbb{R}} k(x-y) H\left(A_{c_{n}}(y)\right) d y+ \\
& +\sum_{i=1}^{\infty} a_{i} \Delta_{i-1} \int_{\mathbb{R}} k\left(x+i c_{n}-y\right) H\left(A_{c_{n}}(y)\right) d y
\end{aligned}
$$

where $A_{c_{n}}$ is the first component of $\mathbf{w}_{c_{n}}(x)$. It is important to note here that the shape of traveling waves are time independent. The subscript on $A_{c_{n}}$ serves to associate the function with the appropriate traveling wave and is NOT used as an index for time.

We now show that the family of functions $\left\{A_{c_{n}}(x)\right\}$ is equicontinuous. Let $\epsilon>0$. Using the fact that $\Delta_{i-1} \leq\left(\rho_{\nu 2}\right)^{i}$ and $a_{i}<1$, we see that the sum $\sum_{i=1}^{\infty} a_{i} \Delta_{i-1}$ is bounded above by the convergent geometric series $\sum_{i=1}^{\infty}\left(\rho_{\nu 2}\right)^{i}$. Hence, choose $M$ so that

$$
\sum_{i=M+1}^{\infty} a_{i} \Delta_{i-1}<\frac{\epsilon}{A_{*}(M+2)}
$$

where we recall that the positive steady-state of the difference equation system $(2.1)$ is $\mathbf{u}_{*}=\left(A_{*}, S_{*}^{0}, S_{*}^{1}, \ldots\right)$.

A result in the proof of Theorem 4.1 in [22] shows that $\lim _{\delta \rightarrow 0} \int_{\mathbb{R}} \mid k(x+\delta)-$ $k(x) \mid d x=0$. So, for $i \in\{0,1, \ldots, M\}$ choose $\delta_{i}$ such that for all $|\delta|<\delta_{i}$,

$$
\int_{\mathbb{R}}\left|k\left(x+\delta+i c_{n}-y\right)-k\left(x+i c_{n}-y\right)\right| d y<\frac{\epsilon}{A_{*}(M+2)} .
$$

Letting $\delta_{*}=\min \left\{\delta_{0}, \delta_{1}, \ldots, \delta_{M}\right\}$, we see that the inequality holds for all $i \in$ $\{0,1, \ldots, M\}$ when $|\delta|<\delta_{*}$. Using this, Eq. (4.37) and the fact that $H\left(A_{c_{n}}(y)\right) \leq$ 
$A_{*}$, we see that

$$
\begin{aligned}
\sum_{i=1}^{\infty} a_{i} \Delta_{i-1} \int_{\mathbb{R}}\left|k\left(x+\delta+i c_{n}-y\right)-k\left(x+i c_{n}-y\right)\right| & H\left(A_{c_{n}}(y)\right) d y \\
& <A_{*}\left(\frac{\epsilon M}{A_{*}(M+2)}+\frac{\epsilon}{A_{*}(M+2)}\right) \\
& =\frac{(M+1)}{M+2} .
\end{aligned}
$$

It follows from this that

$$
\begin{aligned}
\left|A_{c_{n}}\left(x-c_{n}+\delta\right)-A_{c_{n}}\left(x-c_{n}\right)\right|< & \rho_{o}\left|A_{c_{n}}(x+\delta)-A_{c_{n}}(x)\right| \\
& +a_{0} \int_{\mathbb{R}} H\left(A_{c_{n}}(y)\right)|k(x+\delta-y)-k(x-y)| d y \\
& +\frac{\epsilon(M+1)}{M+2} \\
< & \rho_{o}\left|A_{c_{n}}(x+\delta)-A_{c_{n}}(x)\right|+\epsilon \\
\leq & \rho_{o} \sup _{x \in \mathbb{R}}\left|A_{c_{n}}(x+\delta)-A_{c_{n}}(x)\right|+\epsilon .
\end{aligned}
$$

Since this is true for all $x \in \mathbb{R}$ we may take the supremum on the left hand side of the inequality which makes this difference independent of $c_{n}$ and so we obtain

$$
\sup _{x \in \mathbb{R}}\left|A_{c_{n}}(x+\delta)-A_{c_{n}}(x)\right|<\frac{\epsilon}{1-\rho_{o}}
$$

Thus we have that $\left\{A_{c_{n}}(x)\right\}$ is an equicontinuous family of functions.

For fixed $i$ we choose a small positive number $\gamma<A_{*}$. Since $A_{c_{n}}(x)$ is continuous and decreases from $A_{*}$ to 0 , there exists a number $l_{n}$ such that $A_{c_{n}}(x+$ $\left.l_{n}\right)=\gamma$.

Since $A_{c_{n}}\left(x+l_{n}\right)$ is an equicontinuous family of non-increasing functions which are uniformly bounded by $A_{*}$, then by the Arzelá-Ascoli Theorem there exists a subsequence $n_{j}$ such that $A_{c_{n_{j}}}\left(x+l_{n_{j}}\right)$ converges to a non-increasing continuous function $A(x)$ uniformly on every bounded interval.

Taking limits in (4.36), we see that the left-hand side converges to $A\left(x+c^{*}\right)$ since $A_{n_{j}}(x) \rightarrow A(x)$ and $c_{n_{j}} \rightarrow c^{*}$. Also, in view of Lemma 4.6.i and 4.6.iii, the 
right-hand side converges to

$$
\rho_{0} A(x)+a_{0} \int_{\mathbb{R}} k(x-y) H(A(y)) d y+\sum_{i=1}^{\infty} a_{i} \Delta_{i-1} \int_{\mathbb{R}} k\left(x-i c^{*}-y\right) H(A(y)) d y .
$$

Further, $A(0)=\gamma$. It follows that $A$ is a traveling wave solution with speed $c^{*}$, $A(\infty)=0$ and $A(-\infty)=A_{*}$. Also, $A(x)$ is non-increasing since each $A_{c_{n}}(x)$ is monotone.

\section{Non-monotone Case}

We now investigate the existence of traveling wave solutions for the more general case when the growth function $H(u)=F(u) u$ may be non-monotone. In this case $\mathbf{Q}$ may not be order preserving.

Define the operator

$$
Q[u]:=T[u]+R[u]
$$

where

$$
T[u](x):=\rho_{o} u(x)
$$

and

$$
R[u](x):=a_{0} \int_{\mathbb{R}} k(x-y) H(u(y)) d y+\sum_{i=1}^{\infty} a_{i} \Delta_{i-1} \int_{\mathbb{R}} k(x+i c-y) H(u(y)) d y .
$$

Now, define

$$
Q_{c}[u](x):=Q[u](x+c)
$$

We let $Q_{c}^{+}$and $Q_{c}^{-}$be defined by (4.38) and (4.39) with $H$ replaced by $H^{+}$ and $H^{-}$respectively. For all $x$, let $u(x)$ be continuous and $0 \leq u(x) \leq \kappa$. Since $H^{-}(u) \leq H(u) \leq H^{+}(u)$ then

$$
Q_{c}^{-}[u] \leq Q_{c}[u] \leq Q_{c}^{+}[u]
$$

THEOREM 4.7. Let Hypotheses 4.1 and 4.2 be satisfied. Then the follouing hold for the spatial system (4.1): 
1. For any $: \geq c^{*}$ there exists a traveling wave solution $\mathbf{u}_{n}(x)=\mathbf{w}(x-n c)$ with $\mathbf{w}(x) \leq \boldsymbol{\sigma}_{+}$for all $x, \mathbf{w}(\infty)=\mathbf{0}$, and $\lim \inf _{x \rightarrow-\infty} \mathbf{w}(x) \geq \sigma_{-}$.

2. A traveling wave solution $\mathbf{w}(x-n c)$ with $\mathbf{w}(\infty)=0$ and $\lim \inf _{x \rightarrow-\infty} \mathbf{w}(x) \geq$ $\sigma_{-}$does not exist if $c<c^{*}$.

Proof. Case 1: Let $c \geq c^{*}$.

According to (4.35), any traveling wave solution of system (4.1) will satisfy the scalar equation

$$
u(x)=Q_{c}[u](x)
$$

and thus a traveling wave solution is a fixed point of the operator $Q_{c}$.

Because $H^{ \pm}$are monotone functions, Theorem 4.6 says that there exist non-increasing functions $w_{c}^{ \pm}(x)$, such that

$$
\begin{gathered}
Q_{c}^{+}\left[w_{c}^{+}\right](x)=w_{c}^{+}(x), w_{c}^{+}(-\infty)=\kappa, w_{c}^{+}(\infty)=0, \\
Q_{c}^{-}\left[w_{c}^{-}\right](x)=w_{c}^{-}(x), w_{c}^{-}(-\infty)=\sigma_{-}, w_{c}^{-}(\infty)=0 .
\end{gathered}
$$

The following 2 lemmas describe properties about the functions $I, I^{ \pm}$and are taken from [22] so the proofs are omitted.

LEMMA 4.7. The number

$$
l:=\sup \left\{u: 0<u<d \quad \text { and } H(u)<H^{-}(d)\right\}
$$

satisfies the inequalities $0<l \leq d$, and

$$
H^{-}(u)=H^{+}(u)=H(u) \quad \text { for } 0 \leq u \leq l
$$

Proof. See proof in [22].

LEMMA 4.8. If $0<\gamma<l / \kappa$ and if $0<u \leq \kappa$, then $H^{-}(\gamma u) \geq \gamma H^{+}(u)$.

Proof. See proof in [22]. 
Denote the Banach space of bounded continuous functions with the supremum norm as $C(\mathbb{R})$. Let

$$
\begin{array}{r}
E_{c}=\left\{u(x): u \in C(\mathbb{R}), \gamma w_{c}^{+}(x) \leq u(x) \leq w_{c}^{+}(x)\right\} \\
\text { with } 0<\gamma<\min \left\{\frac{A_{-}, l}{\kappa}\right\} .
\end{array}
$$

It is clear that $E_{c}$ is a bounded nonempty closed convex subset of $C(\mathbb{R})$.

LEMMA 4.9. The operator $Q_{c}$ maps $E_{c}$ into $E_{c}$.

Proof. Let $u \in E_{c}$. Then, $u \leq w_{c}^{+}$implies that

$$
Q_{c}[u] \leq Q_{c}^{+}[u] \leq Q_{c}^{+}\left[w_{c}^{+}\right]=w_{c}^{+} .
$$

Alternatively, since $u \geq \gamma w_{c}^{+}$then by Lemma 4.8

$$
Q_{c}[u] \geq Q_{c}^{-}[u] \geq Q_{c}^{-}\left[\gamma w_{c}^{+}\right] \geq \gamma Q_{c}^{+}\left[w_{c}^{+}\right]=\gamma w_{c}^{+} .
$$

Therefore, $Q_{c}[u] \in E_{c}$ and the lemma is proved.

We proceed to prove the theorem.

$Q_{c}$ is of the form $Q_{c}=T_{c}+R_{c}$ where $T_{c}[u](x)=T[u](x+c)$ and $R_{c}[u](x)=$ $R[u](x+c)$. We show that the family of functions $\left\{R_{c}[u](x): u \in E_{c}\right\}$ is equicontinuous in the topology of uniform convergence on bounded intervals. Let $\epsilon>0$ and $u \in E_{c}$. The facts used in the proof of Theorem 4.6 (to show $A_{c_{n}}(x)$ was equicontinuous) apply here. Thus, we choose $M$ so that

$$
\sum_{i=M+1}^{\infty} a_{i} \Delta_{i-1}<\frac{\epsilon}{\kappa(M+2)}
$$

and for $i \in\{0,1, \ldots, M\}$ choose $\delta_{i}$ such that for all $|\delta|<\delta_{i}$,

$$
\int_{\mathbb{R}}|k(x+\delta+i c-y)-k(x+i c-y)| d y<\frac{\epsilon}{\kappa(M+2)} .
$$


Letting $\delta_{*}=\min \left\{\delta_{0}, \delta_{1}, \ldots, \delta_{M}\right\}$, we see that the inequality holds for all $i \in$ $\{0,1, \ldots, M\}$ when $|\delta|<\delta_{*}$. Using this, Eq. (4.41) and the fact that $I(u(y)) \leq$ $H^{+}(u(y)) \leq \kappa$, we see that

$$
\begin{aligned}
\sum_{i=1}^{\infty} a_{i} \Delta_{i-1} \int_{\mathbb{R}}|k(x+\delta+i c-y)-k(x+i c:-y)| & I I(u(y)) d y \\
& <\kappa\left(\frac{\epsilon M}{\kappa(M+2)}+\frac{\epsilon}{\kappa(M+2)}\right) \\
& =\frac{\epsilon(M+1)}{M+2} .
\end{aligned}
$$

It follows from this that

$$
\begin{aligned}
\left|R_{c}[u](x+\delta)-R_{c}[u](x)\right|< & a_{0} \int_{\mathbb{R}} H(u(y))|k(x+\delta-y)-k(x-y)| d y \\
& +\frac{\epsilon(M+1)}{M+2} \\
& <\epsilon .
\end{aligned}
$$

Thus we have that $\left\{R_{c}[u](x): u \in E_{c}\right\}$ is an equicontinuous family of functions which is uniformly bounded by $\kappa$. By the Arzelá-Ascoli Theorem, for any sequence $\left\{\psi_{n}\right\}_{n \geq 1}$ in $R_{c}\left[E_{c}\right]$, there exists a subsequence $n_{j}$ such that $\psi_{n_{j}}(x)$ converges to a continuous function $\psi(x)$ uniformly on every compact subset of $\mathbb{R}$.

This means that $R_{c}\left[E_{c}\right]$ is precompact and so $R_{c}\left[E_{c}\right]$ is contained in a compact set. Further, $T_{c}$ is a $\rho_{o}$-contraction (i.e. $\left\|T_{c} u\right\| \leq \rho_{o}\|u\|$ ). An asymptotic fixed point theorem by Cain and Nashed (pg. 583, Theorem 3.1 [6]) shows that $Q_{c}$ has a fixed point $w_{c}(x) \in E_{c}$ which describes a traveling wave solution to system (4.1).

We have shown that for any $c \geq c^{*}$ the system (4.1) has a traveling wave solution described by the scalar function $w_{c}(x-n c)$ with $\gamma w_{c}^{+}(x) \leq w_{c}(x) \leq w_{c}^{+}(x)$ for all $x$. Since $w_{c}^{+}(x) \leq \kappa$ for all $x$ and $w_{c}^{+}(\infty)=0$, we have that $w_{c}(x) \leq \kappa$ for all $x$ and

$$
w_{c}(\infty)=0
$$


To obtain the behavior of $w_{c}$ at $-\infty$, consider the recursion

$$
u_{n+1}^{-}=Q_{c}^{-}\left[u_{n}^{-}\right]
$$

with the initial condition

$$
u_{0}^{-}(x)=\gamma w_{c}^{+}(x)
$$

We see from Lemma 4.8 that $Q_{c}^{-}\left[u_{0}^{-}\right] \geq u_{0}^{-}$. Thus by induction, $u_{n+1}^{-} \geq u_{n}^{-}$for all $n$ and $u_{n}^{-}(x)$ is non-increasing in $x$. Since $Q_{c}^{-}\left[w_{c}\right] \leq Q_{c}\left[w_{c}\right]=w_{c}$ and $u_{0}^{-} \leq w_{c}$, induction shows that $u_{n}^{-} \leq w_{c}$ for all $n$. This implies that

$$
\liminf _{x \rightarrow-\infty} w_{c}(x) \geq u_{n}^{-}(-\infty) \text { for all } n
$$

We see by Lemma 4.6.ii and 4.6.iv that for any fixed $n$,

$$
u_{n+1}^{-}(-\infty)=\rho_{o} u_{n}^{-}(-\infty)+a_{0} H^{-}\left(u_{n}^{-}(-\infty)\right)+\sum_{i=1}^{\infty} a_{i} \Delta_{i-1} H^{-}\left(u_{n}^{-}(-\infty)\right)
$$

By construction of $\gamma$ we have that $\sigma_{-}>\gamma \kappa=w_{c}^{+}(-\infty)$. Since $u_{n}^{-}(-\infty)$ are non-decreasing, they must converge to the nearest solution of the equation $u=\rho_{o} u+a_{0} H^{-}(u)+\sum_{i=1}^{\infty} a_{i} \Delta_{i-1} H^{-}(u)$. This solution has been defined to be $\sigma_{-}$. Hence,

$$
\liminf _{x \rightarrow-\infty} w_{c}(x) \geq \sigma_{-} .
$$

Case 2: Let $c<c^{*}$. For contradiction let $\mathbf{w}(x)$ be a traveling wave solution with speed $c$ such that $\liminf \operatorname{in}_{x \rightarrow-\infty} \mathbf{w}(x) \geq \sigma_{-}$and $\mathbf{w}(\infty)=\mathbf{0}$. Choose a nonincreasing function $\mathbf{v}_{0}(x)$ such that $\mathbf{v}_{0}(x) \leq \mathbf{w}(x), \mathbf{v}_{0}(x)<\boldsymbol{\sigma}_{-}, \mathbf{v}_{0}(x) \not \equiv 0$ and $\mathbf{v}_{0}(x)=0$ for $x \geq 0$. Let $\mathbf{v}_{n}$ be defined by the recursion $\mathbf{v}_{n+1}=\mathbf{Q}^{-}\left[\mathbf{v}_{n}\right](x)$ where $\mathrm{Q}^{-}$is as described above. By induction and the fact that $\mathbf{Q}^{-}$is order preserving and $\mathbf{Q}^{-} \leq \mathbf{Q}$ we have that

$$
\mathbf{v}_{n}(x) \leq \mathbf{w}(x-n c)
$$

The rest of the proof follows that of Case 2 in Theorem 4.6. 


\section{CHAPTER 5 NUMERICAL SIMULATIONS}

In this chapter we conduct numerical simulations of the spread of a perennial plant population with an age-structured seed bank. For the purpose of these simulations we consider the 4-dimensional finite model described in Chapter 4 Eq.

$$
\begin{aligned}
& A_{(n+1)}(x)=\rho_{o} A_{n}(x)+a_{0} \int_{-\infty}^{\infty} k(x-y) H\left(A_{n}(y)\right) d y+\sum_{j=1}^{2} a_{j} S_{n}^{j-1}(x) \\
& S_{(n+1)}^{0}(x)=s_{0} \int_{-\infty}^{\infty} k(x-y) H\left(A_{n}(y)\right) d y \\
& S_{(n+1)}^{1}(x)=s_{1} S_{n}^{0}(x) \\
& S_{(n+1)}^{2}(x)=s_{2} S_{n}^{1}(x)
\end{aligned}
$$

This is a truncated version of the full spatial model (4.1). Here, the oldest possible age of a seed in the bank is 2 years. We approximate traveling wave solutions to this 4-dimensional finite model (5.1) using an approximation to the convolution integral based on a discrete fast Fourier algorithm motivated by the methods used in $[2,22]$. The algorithm uses the fact that a traveling wave of speed $c>c^{*}$ behaves like a multiple of $e^{-\mu x} \boldsymbol{\xi}(\mu)$ at infinity, where $\mu$ is the smaller root of the equation $\phi(\mu)=c$ with $\boldsymbol{\xi}(\mu)$ the eigenvector of $\mathbf{B}_{\mu}$ corresponding to the positive eigenvalue $\lambda(\mu)$.

We use the Laplace dispersal kernel with mean 0 and variance $2 /\left(200^{2}\right)$ so that

$$
k(|x-y|)=100 e^{-200|x-y|}
$$


and the Ricker function

$$
I(u)=u e^{r-u}
$$

with $r>0$.

The positive solution of the steady-state system

$$
\begin{aligned}
& A=\rho_{o} A+\rho_{d}^{0} \beta^{0} \rho_{\nu 1} F(A) A+\sum_{j=1}^{3} a_{j} S^{j-1} \\
& S^{0}=s_{0} F(A) A \\
& S^{1}=s_{1} S^{0} \\
& S^{2}=s_{2} S^{1}
\end{aligned}
$$

can be calculated from the adult steady-state

$$
\Lambda=r-\ln \left(\frac{1-\rho_{o}}{a_{0}+a_{1} s_{0}+a_{2} s_{1} s_{0}+a_{3} s_{2} s_{1} s_{0}}\right) .
$$

The figures presented here are some approximations to traveling wave solutions for $\left(A, S^{0}, S^{1}, S^{2}\right)$ with the $x$-axis representing position in 1-dimensional space and the $y$-axis representing the density of the species cohort. The viewing window is restricted to the right leading edge of the traveling waves so that long term behavior can be monitored.

All figures use the following color scheme:

- Black line - $A(x)$, Adult plant distribution

- Blue line - $S^{0}(x)$, New seed distribution

- Green line - $S^{1}(x), 1$-year old seed distribution

- Red line - $S^{2}(x), 2$-year old seed distribution

The simulations are organized into groups so that we may investigate three Inain topics: (1) the effect of having an age-dependent germination rate, (2) the effect of having an age-dependent seed bank, and (3) the effect of having a perennial term. Before we dive further into these topics, we make a few general observations about the system which can be seen in Figures 5.1 and 5.3: 
1. Traveling waves for each population cohort take on qualitatively similar shapes. This can be seen by observing that, in any single diagram, all four distributions look very similar.

2. The shape of the traveling waves is related to the growth function parameter $r$. We see that as the parameter $r$ increases, the tail of the traveling waves becomes increasingly unstable.

3. The maximum density of the seed bank populations decrease with age. Recall that the 0-year, 1-year and 2-year old seed bank distributions are represented by the blue, green and red lines, respectively. The maximum seed bank density follows this order as well, with blue the highest, green middle and red the lowest. The adult plant distribution (black line) does not follow a particular pattern. Its position, relative to the seed bank distributions, is related to the choice of survival and germination parameters.

\section{Role of the Germination Rate - Figure 5.1:}

We investigate the role of the age-dependent germination rates $\beta^{0}, \beta^{1}, \beta^{2}$, $\beta^{3}$. We conduct simulations across a wide range of values for $r$ and consider the effect of the germination rate increasing with age, decreasing with age and fluctuating across age groups.

For $r=1$, Figures 5.1a, 5.1b, 5.1c show that the shape of the traveling waves are unaffected by variations in the germination rate. More specifically, when the germination rate increases with age (5.1a), the traveling waves appear to be nonincreasing and this is also true when the germination rate decreases with age $(5.1 \mathrm{~b})$ and varies randomly with age (5.1c). For $r=3$ (Figures 5.1d, 5.1e, 5.1f) and $r=6$ (Figures $5.1 \mathrm{~g}, 5.1 \mathrm{~h}, 5.1 \mathrm{i}$ ) we see that this observation is true, i.e. for fixed $r$, the shape of the traveling waves remain qualitatively the same regardless of the values for the germination rates. We see that the shape of the traveling waves appear 
to be most heavily influenced by the growth function parameter $r$ as is consistent with simulations done for 1-dimensional single species models [21, 22].

Each diagram in Figure 5.1 shows that the maximum density of the seed bank cohorts (blue, green and red lines) decrease with age; this ordering is independent of the germination rates. Alternatively, the maximum density of the adult population relative to the seed bank cohorts depends on the pattern of the germination rates. When germination rate increases with age the adult distribution tends to be in between the new seed distribution and the 2-year old seed distribution as seen in Figures $5.1 \mathrm{a}, 5.1 \mathrm{~d}, 5.1 \mathrm{~g}$. When germination rate decreases with age, Figures $5.1 \mathrm{~b}, 5.1 \mathrm{e}, 5.1 \mathrm{~h}$ show that the maximum adult density is smaller than that of the seed bank cohorts. Not surprisingly, when the germination rates fluctuate with age (Figure 5.1c, 5.1f, 5.1i) there is no observable pattern for adult density relative to seed bank densities.

Finally, it is interesting to note that when $r=6$ (Figures $5.1 \mathrm{~g}, 5.1 \mathrm{~h}, 5.1 \mathrm{i}$ ), the traveling waves for each population cohort display damped oscillations with the peaks occurring in different locations for each cohort. When peaks occur in the other figures $(r=1,3)$ they tend to occur simultaneously. It is not clear why this phenomena occurs. 


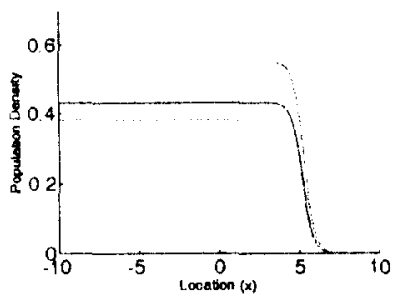

(a) $r=1$

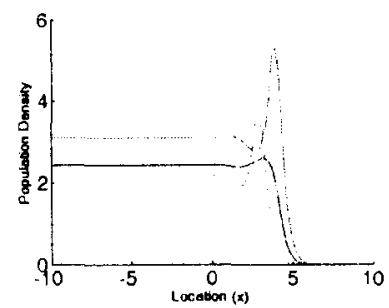

(d) $r=3$

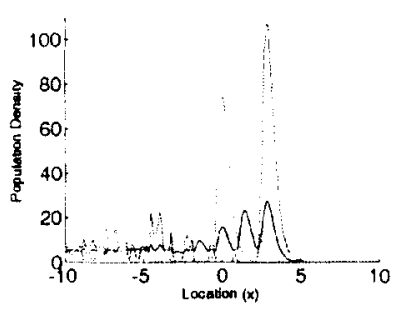

(g) $r=5$

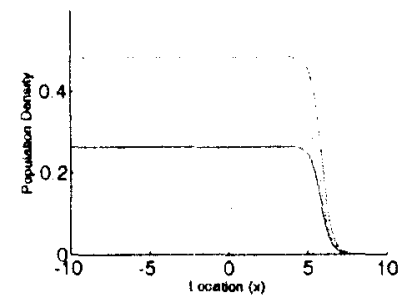

(b) $r=1$

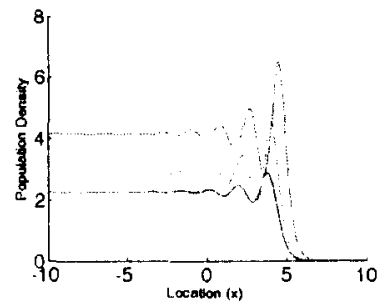

(e) $r=3$

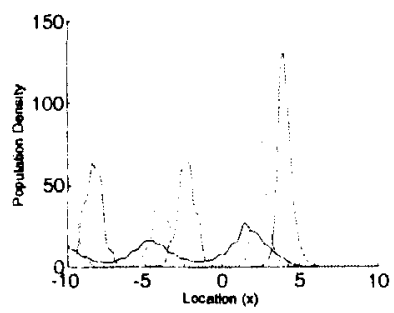

(h) $r=5$

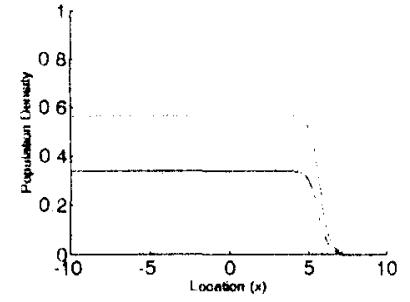

(c) $r=1$

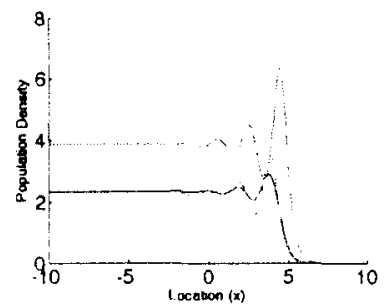

(f) $r=3$

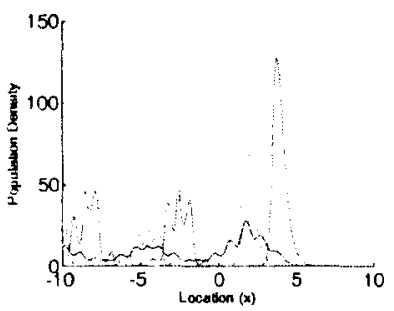

(i) $r=5$

FIGURE 5.1-Role of Germination Rate Numerical simulations for the model with $r=1$ (a, b, c), $r=3(\mathrm{~d}, \mathrm{e}, \mathrm{f})$, and $r=6(\mathrm{~g}, \mathrm{~h}, \mathrm{i})$. Vertically the simulations represent: (1) Leftmost ( $\mathrm{a}, \mathrm{d}, \mathrm{g})$ - traveling waves when the germination rate increases with age, $\beta^{0}=0.025, \beta^{1}=0.05, \beta^{2}=0.1, \beta^{3}=0.2$, (2) Center (b, $\mathrm{e}, \mathrm{h})$ - traveling waves when the germination rate decreases with age, $\beta^{0}=0.2$, $\beta^{1}=0.1, \beta^{2}=0.05, \beta^{3}=0.025$, (3) Rightmost $(\mathrm{c}, \mathrm{f}, \mathrm{i})$ - traveling waves when the germination rate fluctuates with age, $\beta^{0}=0.05, \beta^{1}=0.1, \beta^{2}=0.2, \beta^{3}=0.025$. All other parameters take the following values: $\rho_{o}=0.5, \rho_{d}^{0}=\rho_{d}^{1}=\rho_{d}^{2}=\rho_{d}^{3}=1$, $\rho_{\nu 1}=\rho_{\nu 2}=0.9$. 


\section{Role of the Seed Bank and Perennial Term - Figures 5.2 and 5.3}

The diagrams in Figure 5.2 represent traveling wave solutions when there is no seed bank present (i.e. $s_{i}=0$ for $i=0,1,2$ ) and those in Figure 5.3 represent traveling wave solutions when an age-structured seed bank exists for seeds up to age 2. Both Figures 5.2 and 5.3 follow the same type of flow: Moving across a row from left to right the growth function parameter $r$ is increased $(r=1,3,5)$ and all other parameters are held constant. Moving down a column from top to bottom the perennial survival rate $\rho_{o}$ is increased $\left(\rho_{0}=0,0.25,0.5,0.75\right)$ and all other parameters are held constant. (Note that $\rho_{o}=0$ is the case of an annual plant.) In this way we can observe the effect of the perennial term by comparing diagrams down a column. We can observe the effect of the seed bank by comparing diagrams in Figure 5.2 to those in Figure 5.3 which have the same relative position.

The effect of increasing the perennial survival term $\rho_{o}$ is clear: moving down a column in either Figure 5.2 or 5.3 we see that the distribution increases in magnitude and stabilizes in shape. Thus, a perennial plant with strong survivorship throughout the years will tend to have a more stable and higher density distribution.

By comparing diagrams in Figure 5.2 to those in Figure 5.3 with the same relative position, we see that having a seed bank increases the overall adult plant density (the black distribution in the seed bank Figure 5.3). When $r=1$ (the first column in Figures 5.2 and 5.3) the shape of the adult plant distribution is the same for both the case of having a seed bank and not having a seed bank.

When $r=3$ and the perennial survival term is small (Figures 5.2.b, e and 5.3.b, e) the seed bank has a stabilizing effect on the adult plant density. The graph in Figure 5.2.b representing no seed bank appears to oscillate all the way to $-\infty$ while the graph in Figure 5.3.b representing a seed bank model appears to have oscillations which are damped for large negative $x$. 
When $r=3$ and the perennial survival term is large (Figures $5.2 . \mathrm{h}, \mathrm{k}$ and 5.3.h, k) the shape of the traveling waves are similar for both the case of having a seed bank and not having one. Thus, when the perennial survival rate is large enough, the stabilizing effect of this term overpowers the effects of the seed bank and creates a distribution for both the seed bank and no seed bank which are similar in shape. The patterns just discussed for the case of $r=3$ can be seen for the case when $r=6$ as well.

In general, both the perennial term and the seed bank can have a stabilizing effect on the adult plant distribution and increase the maximum plant density. We also observe that when the perennial term is large enough, it can overpower the effect of the seed bank, making the distributions similar when the seed bank is either present or absent. 


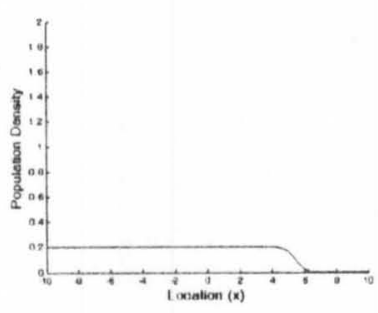

(a) $r=1, \rho_{o}=0$

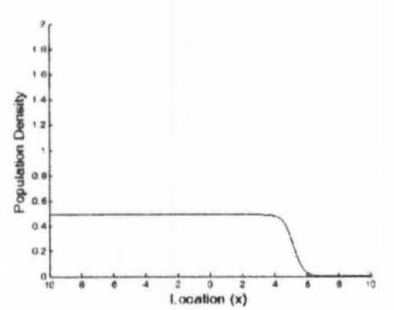

(d) $r=1, \rho_{o}=0.25$

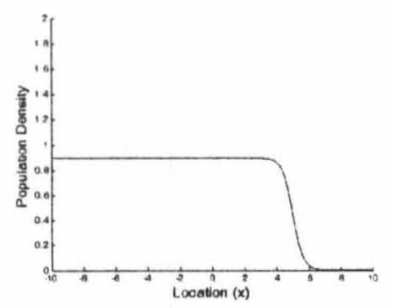

(g) $r=1, \rho_{o}=0.5$

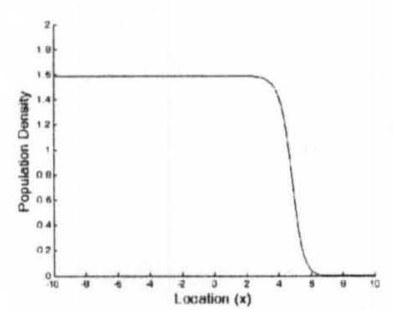

(j) $r=1, \rho_{o}=0.75$

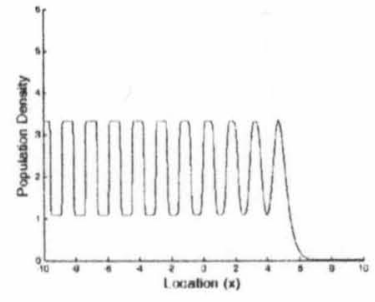

(b) $r=3, \rho_{o}=0$

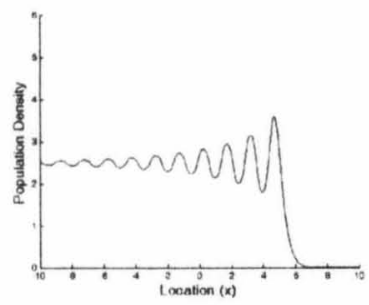

(e) $r=3, \rho_{o}=0.25$

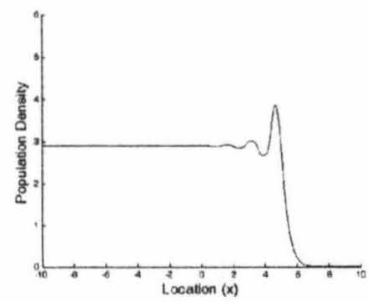

(h) $r=3, \rho_{o}=0.5$

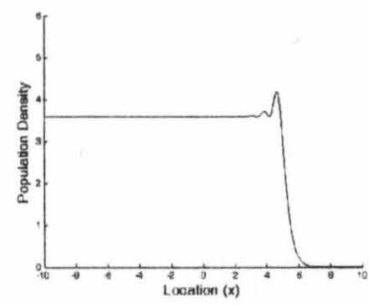

(k) $r=3, \rho_{o}=0.75$

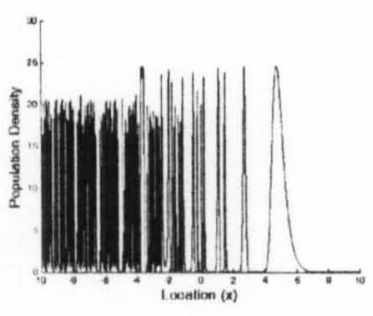

(c) $r=5, \rho_{o}=0$

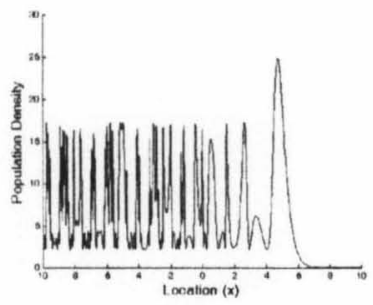

(f) $r=5, \rho_{o}=0.25$

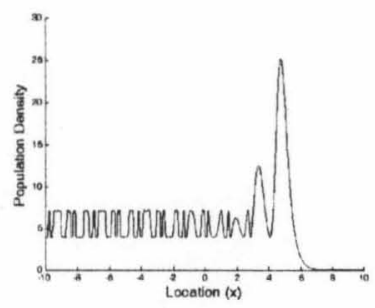

(i) $r=5, \rho_{o}=0.5$

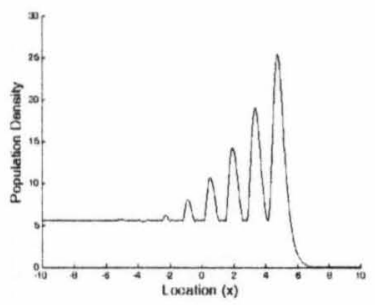

(l) $r=5, \rho_{o}=0.75$

FIGURE 5.2-No Seed Bank Numerical simulations for the model when $s_{i}=0$ for $i=0,1,2$. 


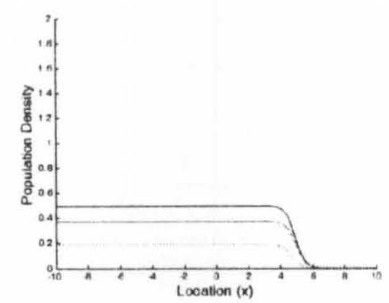

(a) $r=1, \rho_{o}=0$

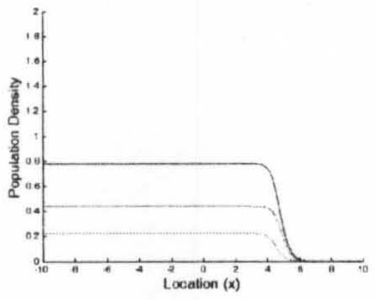

(d) $r=1, \rho_{o}=0.25$

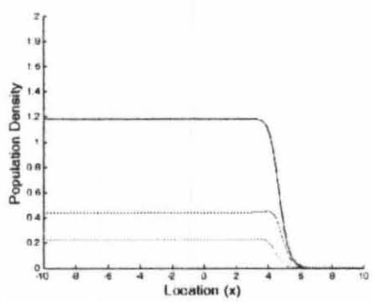

(g) $r=1, \rho_{o}=0.5$

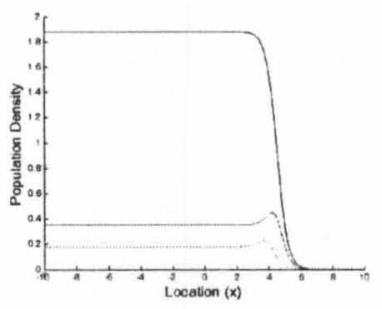

(j) $r=1, \rho_{o}=0.75$

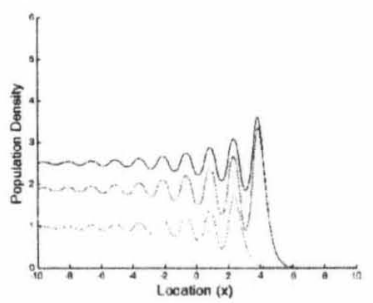

(b) $r=3, \rho_{o}=0$

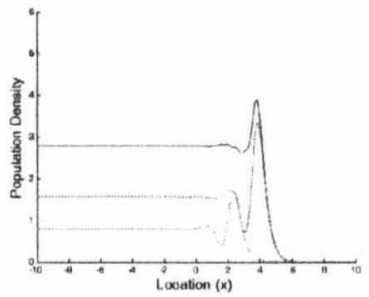

(e) $r=3, \rho_{o}=0.25$

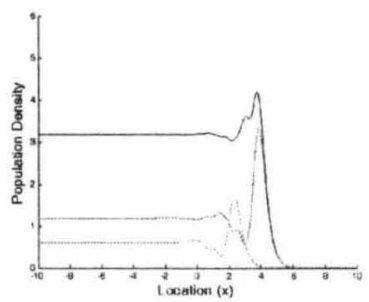

(h) $r=3, \rho_{o}=0.5$

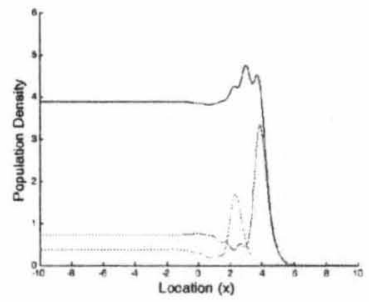

(k) $r=3, \rho_{o}=0.75$

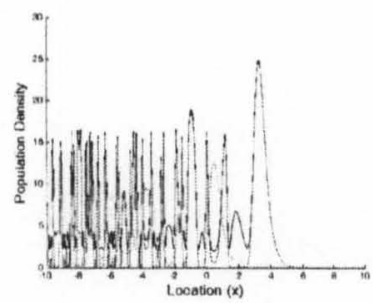

(c) $r=5, \rho_{o}=0$

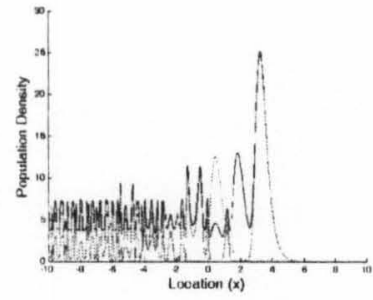

(f) $r=5, \rho_{o}=0.25$

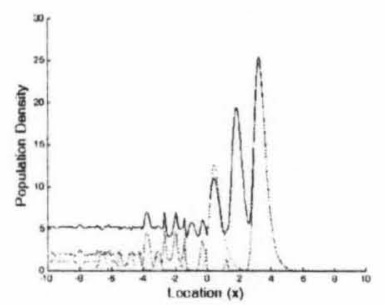

(i) $r=5, \rho_{o}=0.5$

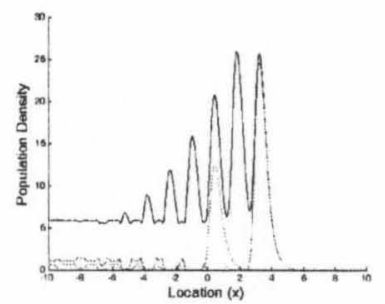

(1) $r=5, \rho_{o}=0.75$

FIGURE 5.3-Seed Bank Numerical simulations for the model with an agestructured seed bank for seeds up to age 2: $A(x)=$ black line, $S^{0}(x)=$ blue line, $S^{1}(x)=$ green line, $S^{2}(x)=$ red line. 


\section{CHAPTER 6 CONCLUSION AND FUTURE DIRECTIONS}

\subsection{Conclusion}

In this dissertation we formulated a system of integro-difference equations which serve to model growth and spread of perennial and annual plants with an age-structured seed bank. This model is an extension of Li's seed bank model for annual plants recently published in the Journal of Mathematical Biology [21]. There is no age-structuring of the seed bank in that model. The advantage of our model over current spatial plant models is that we distinguish between ages of seeds in the bank and we include a perennial term which permits the survival of adult plants from year to year. To the best of our knowledge, this is the first model involving infinitely many integro-difference equations. The underlying dynarmics are very complicated when the growth function is non-monotone however we can still give a complete description of the spreading speed and traveling wave solutions.

In Chapter 3 we analyzed the non-spatial system (3.1). We proved the existence of an eigenvalue for this system linearized about the origin and provide a condition for when the origin is unstable. We showed that the system has a strictly positive steady-state and that a certain class of solutions converge to this steady-state when the origin is unstable. Bifurcation diagrams, utilizing the Ricker function, are included to numerically simulate the model's behavior when; (1) the growth function parameter is varied and (2) the new seed germination rate is varied. 
The Ricker function has been used to study plant populations (see [19, 34]). When the growth function parameter is varied the bifurcations are reminiscent of the well studied logistic growth with stable solutions, moving to period doubling and then to chaos. Of particular interest, however, are the bifurcation diagrams when the new seed germination rate $\beta^{0}$ is varied. Several diagrams display that when $\beta^{0}$ is close to 0 the system grows in a chaotic manner. As $\beta^{0}$ is increased, the system stabilizes, experiences period doubling, becomes chaotic, stabilizes again and repeats this pattern until $\beta^{0}$ gets close to 1 . This type of switching behavior is something really new and is of interest for future studies.

The spatial system (4.1) is analyzed in Chapter 4. We establish the existence and properties of a positive eigenvalue and eigenvectors for the linearized moment generating matrix $\mathbf{B}_{\mu}$. We show that this positive eigenvalue $\lambda_{*}(\mu)$ is related to the spreading speed $c^{*}$ of the system under certain initial conditions in the following way:

$$
c^{*}=\inf _{\mu>0} \frac{\ln \lambda_{*}(\mu)}{\mu}
$$

Further, we proved the existence of traveling wave solutions when $c \geq c^{*}$ and showed that traveling wave solutions do no exist when $c<c^{*}$. This shows that the spreading speed can be characterized as the slowest speed of a class of traveling wave solutions.

In Chapter 5 we conduct numerical simulations of traveling wave solutions for a 4-dimensional truncated version of the model so that seeds in the bank can only survive up to 2 years. The Ricker function (1.2) is used as the growth function. From these simulations we observe that the tail of traveling wave solutions can take on many different patterns, determined in large part by the value of the growth function parameter $r$. We also see that the seed bank and the perennial term can have a stabilizing effect on the traveling waves. Additionally, when the perennial term is large enough, the stabilizing effect of this term overpowers the 
effect of the seed bank effectively making the seed bank vs. no seed bank models indistinguishable.

\subsection{Future Directions}

This model can be extended in several different directions:

1. Permit density-dependent survival and germination parameters to account for crowding. Crowding describes the effect that high population density has on the reproductive output and adaptive strategies of ecological populations. It is known that crowding in plant populations oftentimes has a negative effect on reproductive output [39]. Additionally, it has also been shown that crowding can cause insects to have a more detrimental effect on the crowded plants [15]. By permitting the survival and germination parameters in (4.1) to be non-linear (or density-dependent) we may study the effects of crowding and how it relates to the spatial spread of perennial plant populations.

2. Consider stochastic fluctuations in population growth. Environmental variability including climate effects, effects of human interaction and effects of competitors are stochastic. One year may see booming reproduction due to a particularly agreeable climate while the next year may bring drought or flooding resulting in a large number of deaths and low reproductive output. Presently our model assumes the environment is temporally constant, using a specific function $H(A)=F(A) A$ to model growth. We may replace the growth function with a stochastic variable to model these random effects similar to the approaches used in $[33,44]$.

3. Consider an Allee effect. An Allee effect refers to the observed effect that "under-crowding" or low population densities correspond with low reproduc- 
tion or decreased fitness [41]. There are many reasons that a population might exhibit this Allee effect including an individuals inability to replace itself in low densities ("pollen limitation"), inbreeding depression, and more [20]. We may incorporate this effect into our model (4.1) by replacing the requirement that the growth function is bounded above by its linearization $\left(H(A) \leq H^{\prime}(0) A\right)$, with a weaker condition.

4. Consider two-species model. Analyzing how species interact and compete upon invasion has been of long standing interest in ecology. This knowledge has the potential to aid policy concerning pest control and minimization of disease spread [7]. By coupling two systems of the form (4.1) we may study species interaction and examine the speed at which the invading species spreads into its competitors habitat.

\section{Add further structure to model woody plants and other perennial} plants with juveniles. Many plants, including some woody plants, go through a juvenile stage post-germination before maturing into a fully reproductive adult. To model this type of situation we could add an intermediate cohort between the seed bank and the adult populations to monitor the juvenile population. We would assume juveniles to be sterile and immobile. Juveniles could only contribute to either the adult population through maturation, or back to the juvenile population if the plant is not fully matured at the end of a yearly cycle.

\section{Acquire field data to test model and fit parameters.}




\section{REFERENCES}

[1] Edward J. Allen, Linda J. S. Allen, and Xiaoning Gilliam, Dispersal and competition models for plants, J. Math. Biol. 34 (1996), no. 4, 455-481.

[2] M. Andersen, Properties of some density-dependent integro-difference equation population models, Math. Biosci. 104 (1991), 135--157.

[3] M. Begon, J. Harper, and C. Townsend, Ecology: individuals, populations, and communities, second ed., Blackwell Scientific Publications, Boston, 1990.

[4] R. Beverton and S. Holt, On the dynamics of exploited fish populations, series 2, vol. 19 ed., Fisheries Investigations, London, 1957.

[5] J. S. Brown and D. L. Venable, Evolutionary ecology of seed-bank annuals in temporally varying environments, (1986).

[6] G. L. Cain, Jr. and M. Z. Nashed, Fixed points and stability for a sum of two operators in locally convex spaces, Pacific J. Math. 39 (1971), 581-592.

[7] G. R. Conway, Mathematical models in applied ecology: review article, Nature 269 (1977), 291-297.

[8] M. J. Crawley, Plant ecology, second ed., University Press, Cambridge, 1997.

[9] D. P. Hardin, P. Takávc, and G. F. Webb, Asymptotic properties of a continuous-space discrete-time population model in a random environment, J. Math. Biol. 26 (1988), no. 4, 361-374. 
[10] _ Dispersion population models discrete in time and continuous in space, J. Math. Biol. 28 (1990), no. 1, 1-20.

[11] J. L. Harper, Population biology of plants, Academic Press, New York, 1977.

[12] Deborah R. Hart and Robert H. Gardner, A spatial model for the spread of invading organisms subject to competition, J. Math. Biol. 35 (1997), no. 8, 935-948.

[13] M. P. Hassell, Density-dependence in single-species populations, J. Anim. Ecol. 44 (1975), 283-295.

[14] A. Hastings and K. Higgins, Persistence of transients in spatially structured ecological models, Science 263 (1994), 1133-1136.

[15] R. Karban, A. Brody, and W. Schnathorst, Crowding and a plant's ability to defend itself against herbivores and diseases., The American Naturalist 134 (1989), no. 5, 749-760.

[16] Mark Kot, Discrete-time travelling waves: ecological examples, J. Math. Biol. 30 (1992), no. 4, 413-436.

[17] _ Elements of mathematical ecology, University Press, Cambridge, 2001.

[18] Mark Kot and William M. Schaffer, Discrete-time growth-dispersal models, Math. Biosci. 80 (1986), no. 1, 109-136.

[19] J. Kuang and P. Chesson, Coexistence of annual plants: generalist seed predation weakens the storage effect., Ecology 90 (2009), 170-182.

[20] M. A. Lewis and P. Kareiva, Allee dynamics and the spread of invading organisms, Theor. Popul. Biol. 43 (1993), 141-158. 
[21] Bingtuan Li, Traveling wave solutions in a plant population model with a seed bank, J. Math. Biol. (2011).

[22] Bingtuan Li, Mark A. Lewis, and Hans F. Weinberger, Existence of traveling waves for integral recursions with nonmonotone growth functions, J. Math. Biol. 58 (2009), no. 3, 323-338.

[23] Bingtuan Li, Hans F. Weinberger, and Mark A. Lewis, Spreading speeds as slowest wave speeds for cooperative systems, Math. Biosci. 196 (2005), no. 1, $82-98$.

[24] Roger Lui, A nonlinear integral operator arising from a model in population genetics. I. Monotone initial data, SIAM J. Math. Anal. 13 (1982), no. 6, 913-937.

[25] _ A nonlinear integral operator arising from a model in population genetics. II. Initial data with compact support, SIAM J. Math. Anal. 13 (1982), no. $6,938-953$.

[26] __ Existence and stability of travelling wave solutions of a nonlinear integral operator, J. Math. Biol. 16 (1982/83), no. 3, 199-220.

[27]__ Biological growth and spread modeled by systems of recursions. I. Mathematical theory, Math. Biosci. 93 (1989), no. 2, 269-295.

[28] N. MacDonald and A. R. Watkinson, Models of an annual plant population with a seedbank, J. Theoret. Biol. 93 (1981), no. 3, 643-653.

[29] R. M. May, Biological populations obeying difference equations: Stable points, stable cycles, and chaos, J. Theor. Biol. 49 (1975), 511-524.

[30] R. M. May and G. F. Oster, Bifurcations and dynamic complexity in simple ecological models, Am. Nat. 110 (1976), 573-599. 
[31] M. Neubert and H. Caswell, Demography and dispersal: calculation and sensitivity analysis of invasion speed for structured populations, Ecology 81 (2000), 1613-1628.

[32] M. Neubert, M. Kot, and M. A. Lewis, Dispersal and pattern formation in a discrete-time predator-prey model, Theor. Popul. Biol. 48 (1995), 7-43.

[33] __ Invasion speeds in fluctuating environments, Proc. R. Soc. Lond. 267 (2000), 1603-1610.

[34] J.M. Norghauer and D. M. Newbery, Recruitment limitation after mastseeding in two african rain forest trees, Ecology 91 (2010), 2303-2312.

[35] Stephen W. Pacala, Neighborhood models of plant population dynamics. IV. Single species and multispecies models of annuals with dormant seeds, Am. Nat. 128 (1986), 859-878.

[36] A. G. Pakes and R. A. Maller, Mathematical ecology of plant species competition: a class of deterministic models for binary mixtures of plant genotypes, University Press, Cambridge, 1990.

[37] M. Rees and M.J. Long, The analysis and interpretation of seedling recruitment curves, Am. Nat. 141 (1993), 233-262.

[38] K. Ritland, The joint evolution of seed dormancy and flowering time in annual plants living in variable environments, Theor. Popul. Biol. 24 (1983), 213-243.

[39] J. Sarukhan, Studies on plant demography: ranunculus repens l., r. bulbosus l. and $r$. acris $l$. ii. reproductive strategies and seed population dynamics, Journal of Ecology 62 (1974), 151-177.

[40] K. P. Schmidt and L. R. Lawlor, Growth rate projection and life history sensitivity for annual plants with a seed bank, Am. Nat. 121 (1983), 525-539. 
[41] A. Stephens, What is the allce effect?, Oikos 87 (1999), 185-190.

[42] A. R. Templeton and D. A. Levin, Evolutionary consequences of seed pools, Am. Nat. 114 (1979), 232-249.

[13] H. R. Thieme, Density-dependent regulation of spatially distributed populations and their asymptotic speed of spread, J. Math. Biol. 8 (1979), 173-187.

[44] A. Valleriani and K. Tielborger, Effect of age on germination of dormant seeds, Theoretical Population Biology 70 (2006), 1-9.

[45] A. R. Watkinson, Density-dependence in single-species populations of plants, J. Theor. Biol. 83 (1980), 345-357.

[46] H. F. Weinberger, Asymptotic behavior of a model in population genetics, Nonlinear partial differential equations and applications (Proc. Special Sem., Indiana Univ., Bloomington, Ind., 1976-1977), Springer, Berlin, 1978, pp. 4796. Lecture Notes in Math., Vol. 648.

[47] _ Long-time behavior of a class of biological models, SIAM J. Math. Anal. 13 (1982), no. 3, 353-396.

[48] Hans F. Weinberger and Bingtuan Li, Spreading speeds and traveling waves for almost cooperative multispecies discrete-time recursions, pre-press (2010). 


\section{CURRICULUM VITAE}

\section{Kimberly I. Meyer}

\section{Education}

University of Louisville, Louisville, KY

Ph.D., Applied and Industrial Mathematics, expected Spring 2012

M.A., Mathematics, May 2008

B.S., Mathematics, May 2007

\section{Professional Experience}

\section{Actuarial Intern}

Summer 2011

Milliman Denver Health Practice, Denver, CO

Graduate Teaching Assistant, University of Louisville

Math 111: College Algebra, Recitation Instructor

Aug. 2010 - May 2011

Math 111: College Algebra, Recitation Instructor

Aug. 2007 - May 2008

Research Assistant

Summer 2009

University of Louisville, Louisville, $\mathrm{KY}$

National Science Foundation GK-12 Fellow

Aug. 2008 - June 2009

University of Louisville, Louisville, KY

Research Experience for Undergraduate Students

Texas Tech University, Lubbock, TX

Private Tutor and University of Louisville Math Lab Tutor

Louisville, KY

Aug. 2004 - June 2010

\section{Talks \& Presentations}

The Spreading Speed and Traveling Wave Solutions for a Competition Model.

Workshop for Young Researchers in Math Biology, Columbus, OH. Sept. 2010. Graduate Student Seminar, University of Louisville, Louisville, KY. Jan. 2010. Math Biology Seminar, University of Louisville, Louisville, KY. $\quad$ Nov. 2009. The 2nd International Conference on Mathematical Modeling and Analysis of Populations in Biological Systems, Huntsville, AL.

Oct. 2009.

Complex Analysis and Dynamics of Polynomial Hele-Shaw Cells.

The Joint Mathematics Meetings, San Diego, CA.

Jan. 2008.

Annual Mathematics Symposium, Bowling Green, KY.

Oct. 2007. 
The Devil's Staircase.

Conference for Underyraduate Women in Math, Lincoln, NE.

Feb. 2007.

Small Subsets of the Real Line.

Conference for Underyraduate Women in Math, Lincoln, NE.

Feb. 2006.

\section{Awards}

University Fellowship, University of Louisville, Alig. 2009 - May 2012 Southeastern Actuaries Conference Scholarship, Aıt. 2010 - May 2011 Provost Hallmark Award, University of Louisville, Aıt. 2003 - May 2007 William Bullitt Scholarship, University of Louisville, 2006 Robert J. Bickel Scholarship, University of Louisville,

\section{Service}

TEDxUofL - independently organized TED event

Coordinator, Budget and Programming.

Jan. 2012 - March 2012

Arts and Sciences Graduate Student Union

Information Officer.

Representative.

Graduate Student Council

Treasurer and Events Committee Chair.

Representative.

Aug. 2011 - . May 2012 Aug. 2010 - May 2011

Louisville Regional Science Fair Judge

Aug. 2010 - May 2011

Aug. 2011 - May 2012

Aug. 2009 - May 2010

2009, 2010, 2011, 2012

Every 1 Reads Volunteer

2009

University of Louisville Math Club

Vice President.

Aug. 2005 - May 2007

Participant.

Aug. 2004 - May 2005

\section{Professional memberships}

Mathematical Association of America

American Mathematical Society

Since 2007

Since 2007 